TRANSACTIONS OF THE

AMERICAN MATHEMATICAL SOCIETY

Volume 362, Number 10, October 2010, Pages 5411-5453

S 0002-9947(2010)04966-9

Article electronically published on May 25, 2010

\title{
GALOIS THEORY FOR ITERATIVE CONNECTIONS AND NONREDUCED GALOIS GROUPS
}

\author{
ANDREAS MAURISCHAT
}

\begin{abstract}
This article presents a theory of modules with iterative connection. This theory is a generalisation of the theory of modules with connection in characteristic zero to modules over rings of arbitrary characteristic. We show that these modules with iterative connection (and also the modules with integrable iterative connection) form a Tannakian category, assuming some nice properties for the underlying ring, and we show how this generalises to modules over schemes. We also relate these notions to stratifications on modules, as introduced by A. Grothendieck (cf. Berthelot and Ogus, 1978) in order to extend integrable (ordinary) connections to finite characteristic. Over smooth rings, we obtain an equivalence of stratifications and integrable iterative connections. Furthermore, over a regular ring in positive characteristic, we show that the category of modules with integrable iterative connection is also equivalent to the category of flat bundles as defined by D. Gieseker in 1975 .

In the second part of this article, we set up a Picard-Vessiot theory for fields of solutions. For such a Picard-Vessiot extension, we obtain a Galois correspondence, which takes into account even nonreduced closed subgroup schemes of the Galois group scheme on one hand and inseparable intermediate extensions of the Picard-Vessiot extension on the other hand. Finally, we compare our Galois theory with the Galois theory for purely inseparable field extensions given by S. Chase in 1976 .
\end{abstract}

\section{INTRODUCTION}

For characteristic zero, N. Katz described in Kat87 a general setting of modules with connection to describe partial linear differential equations, and established a Galois theory from an abstract point of view: He showed that - under some assumptions on the ring - the category of modules with connection (and also that of modules with integrable connection) forms a neutral Tannakian category over the field of constants, and neutral Tannakian categories are known to be equivalent to categories of finite dimensional representations of proalgebraic groups (see [DM89]). However, this theory works only in characteristic zero. This is mainly caused by the fact that in positive characteristic $p$, every $p$-th power of an element in a ring is differentially constant. A. Grothendieck gave a notion of stratifications (cf. [BO78]) which generalises the notion of integrable connections to arbitrary characteristic and which turns out to be a "good" category. In positive characteristic, a theorem of Katz (see [Gie75]) shows that over smooth schemes, modules with stratifications are

Received by the editors February 22, 2008 and, in revised form, October 31, 2008.

2000 Mathematics Subject Classification. Primary 13N99; Secondary 12H20, 12F15.

Key words and phrases. Galois theory, differential Galois theory, inseparable extensions, connections. 
equivalent to flat bundles (or F-divided sheaves as they are called in [San07]), which enables Gieseker and Dos Santos to obtain further properties of the fundamental group scheme, resp. the Tannakian group scheme.

In the first part of this article, we set up a theory over rings of arbitrary characteristic, which generalises the characteristic zero setting not only in the integrable case, but also in the nonintegrable case, using so-called iterative connections. The integrable version, however (so-called modules with integrable iterative connection), is again equivalent to flat bundles over a regular ring in positive characteristic (cf. Section (8).

To obtain this theory differentials will be replaced by a family of higher differentials, similar to the step from derivations to higher/iterative derivations in positive characteristic (see for example Mat01] and MvdP03]). In getting the right setting, the main idea is the following: For an algebra $R$ over a perfect field $K$, regard an iterative derivation on $R$ over $K$ (or more generally, a higher derivation), not as a sequence of $K$-linear maps $\left(\partial^{(k)}: R \rightarrow R\right)_{k \in \mathbb{N}}$ (as it is done in HS37, Mat01, etc.) but as a homomorphism of $K$-algebras $\psi: R \rightarrow R[[T]]$ by summing up, in detail $\psi(r):=\sum_{k=0}^{\infty} \partial^{(k)}(r) T^{k}$ ( $\psi$ is often called the Taylor series). Moreover, regard the ring of power series $R[[T]]$ as a completion of the graded $R$-algebra $R[T]$. This leads to the notion of "cgas" (completions of graded algebras; cf. Section 2), which allows us to generalise the definition of a higher derivation and to obtain a universal object $\hat{\Omega}_{R / K}$ with a universal higher derivation $\mathrm{d}_{R}: R \rightarrow \hat{\Omega}_{R / K}$, replacing the module of differentials $\Omega_{R / K}$ used in the classical theory (cf. Theorem 3.10).

In Section 4, we introduce the definition of a higher connection on an $R$-module. Furthermore, we show that a finitely generated $R$-module that admits such a higher connection is locally free if $R$ is regular and is a finitely generated $K$-algebra (Corollary 4.5). At least in positive characteristic, this is an improvement to the literature, since no integrability condition is needed. Although modules with higher connection might be interesting on their own, our main concern are modules with so-called iterative connection and modules with integrable iterative connection (cf. Section 5), which are obtained by requiring additional properties on the higher connection. One of the main results of the first part is given in Section [6 namely

Theorem 6.10, Let $R$ be a regular ring over a perfect field $K$ and the localisation of a finitely generated $K$-algebra, such that $\operatorname{Spec}(R)$ has a $K$-rational point. Then the categories $\operatorname{HCon}(R / K), \mathbf{I C o n}(R / K)$ and $\mathbf{I C o n}_{\mathbf{i n t}}(R / K)$ of $R$-modules with higher connection, resp. iterative connection, resp. integrable iterative connection, are neutral Tannakian categories over $K$.

The reason for considering iterative and integrable iterative connections becomes clear in the next two sections. In Section 7 , we have a look at characteristic zero. Here we show that iterative connections on modules are in one-to-one correspondence to ordinary connections, if $R$ is regular, and that the integrability conditions coincide via this correspondence. Hence the theory of modules with (integrable) iterative connection really is a generalisation of modules with (integrable) connection in characteristic zero.

Section 8 is dedicated to the case of positive characteristic. The main result here is the equivalence between the category $\operatorname{ICon}_{\text {int }}(R / K)$ and the category of Frobenius compatible projective systems (Fc-projective systems) over the ring $R$. (Again under the assumption that $R$ is regular.) Essentially, Fc-projective systems over $R$ can be identified with flat bundles over $\operatorname{Spec}(R)$, resp. F-divided sheaves on 
$\operatorname{Spec}(R)$. Using the equivalence above, we can deduce from Corollary 4.5 that for an Fc-projective system $\left\{M_{i}\right\}_{i \in \mathbb{N}}$, the $R$-module $M_{0}$ is locally free. This is a slight improvement of [San07, Lemma 6, where the underlying field $K$ is supposed to be algebraically closed.

As mentioned in the beginning, stratifications on modules as introduced by A. Grothendieck (cf. BO78]) also generalise the notion of integrable (ordinary) connections. At least if $R$ is smooth over $K$ and $K$ is algebraically closed, in characteristic zero as well as in positive characteristic, we can deduce from our results that the category of stratified modules and the category $\mathbf{I C o n}_{\mathbf{i n t}}(R / K)$ are equivalent, using the equivalence between stratifications and integrable connections on modules in characteristic zero (cf. [BO78], Thm. 2.15), respectively a theorem of Katz on the equivalence of stratified modules and flat bundles in positive characteristic (cf. [Gie75], Thm. 1.3). However, there is no obvious direct correspondence between stratifications and integrable iterative connections, and it is an open question whether there is any correspondence at all if $R$ is not smooth.

We conclude the first part of this paper by outlining a generalisation of modules with higher connection to sheaves of modules with higher connection (resp. (integrable) iterative connection) over schemes in Section 9 .

In the second part (Sections 10, 11 and 12), we consider solution rings and solution fields for modules with iterative connection, which we call pseudo-PicardVessiot rings (PPV-rings), resp. pseudo-Picard-Vessiot fields (PPV-fields), following the notion of classical differential Galois theory. Indeed, the Picard-Vessiot theory given here is set up in a more general context (namely for so-called $\theta$-fields), so that it can be applied not only to modules with iterative connection, but also to the iterative differential modules as in Mat01 and MvdP03. Given such a PPVring $R$ over a $\theta$-field $F$, we obtain a Galois group scheme $\mathcal{G}:=\operatorname{Gal}(R / F)$ defined over the constants $C_{F}$ of $F$ (cf. Proposition 10.9) and we show that $\operatorname{Spec}(R)$ is a $\left(\mathcal{G} \times{ }_{C_{F}} F\right)$-torsor (cf. Corollary 10.11). The main theorem of this part is the Galois correspondence, namely

Theorem 11.5 (Galois correspondence). Let $R$ be a PPV-ring over some $\theta$-field $F, E:=\operatorname{Quot}(R)$ be the quotient field of $R$ and $\mathcal{G}:=\underline{\mathrm{Gal}}(R / F)$ be the Galois group scheme of $R / F$.

i) There is an anti-isomorphism of the lattices

$$
\mathfrak{H}:=\{\mathcal{H} \mid \mathcal{H} \leq \mathcal{G} \text { closed subgroup schemes of } \mathcal{G}\}
$$

and

$$
\mathfrak{M}:=\{M \mid F \leq M \leq E \text { intermediate } \theta \text {-fields }\}
$$

given by $\Psi: \mathfrak{H} \rightarrow \mathfrak{M}, \mathcal{H} \mapsto E^{\mathcal{H}}$ and $\Phi: \mathfrak{M} \rightarrow \mathfrak{H}, M \mapsto \underline{\mathrm{Gal}}(R M / M)$.

ii) If $\mathcal{H} \leq \mathcal{G}$ is normal, then $E^{\mathcal{H}}=\operatorname{Quot}\left(R^{\mathcal{H}}\right)$ and $R^{\mathcal{H}}$ is a PPV-ring over $F$ with Galois group scheme $\operatorname{Gal}\left(R^{\mathcal{H}} / F\right) \cong \mathcal{G} / \mathcal{H}$.

iii) If $M \in \mathfrak{M}$ is stable under the action of $\mathcal{G}$, then $\mathcal{H}:=\Phi(M)$ is a normal subgroup scheme of $\mathcal{G}, M$ is a PPV-extension of $F$ and $\underline{\mathrm{Gal}}(R \cap M / F) \cong$ $\mathcal{G} / \mathcal{H}$

iv) For $\mathcal{H} \in \mathfrak{H}$, the extension $E / E^{\mathcal{H}}$ is separable if and only if $\mathcal{H}$ is reduced.

Here, $R^{\mathcal{H}}$, resp. $E^{\mathcal{H}}$, denote functorial invariants of $R$, resp. $E$, under the action of the group functor $\mathcal{H}$ (cf. Section 11).

Contrary to the Galois correspondence given by Matzat and van der Put in MvdP03 in the iterative differential case, our correspondence takes into account 
not only reduced subgroup schemes and intermediate iterative differential fields over which $E$ is separable 1 but even the nonreduced subgroup schemes and those intermediate fields over which $E$ is inseparable. By part iv) of the theorem, in this general setting also the separability condition and the reducedness condition correspond to each other. Our Galois correspondence is quite similar to a Galois correspondence given by M. Takeuchi for so-called C-ferential fields between intermediate C-ferential fields and closed subgroup schemes, although he uses a different definition of PV-extension. The relation to this correspondence is discussed in Remark 11.1. We conclude Section [1] with some examples to enlight our Galois correspondence (cf. Example 11.8).

In the last section, the Galois theory given there is compared with the Galois theory for purely inseparable field extensions given by S. Chase in Cha76, who extended the theory of N. Jacobson (cf. [Jac64]) to Galois group schemes of arbitrary exponents.

\section{Notation}

Throughout this article, $K$ denotes a perfect field, $R$ and $\tilde{R}$ denote integral domains, which are finitely generated $K$-algebras (or localisations of finitely generated $K$-algebras) and $f: R \rightarrow \tilde{R}$ denotes a homomorphism of $K$-algebras. $M$ will be a finitely generated $R$-module.

As mentioned in the Introduction, we need the notion of "completions of graded algebras". So let $\bigoplus_{i=0}^{\infty} B_{i}$ be a graded $R$-algebra. Then the ideals $I_{k}:=\bigoplus_{i=k}^{\infty} B_{i}$ form a filtration of the algebra, and one obtains a completion of $\bigoplus_{i=0}^{\infty} B_{i}$ with respect to this filtration (cf. Eis95, Ch. 7.1). As an $R$-module, this completion is isomorphic to $\prod_{i=0}^{\infty} B_{i}$.

Definition 2.1 (cgas). A commutative $R$-algebra $B$ is called a completion of a graded algebra, or a cga for short if $B$ is the completion of a graded $R$-algebra $\bigoplus_{i=0}^{\infty} B_{i}$ in the above sense. We call $B_{i}$ the $i$-th homogeneous component of $B$. $B$ is called a $\tilde{R}$-cga if $B$ is a cga with $B_{0}=\tilde{R}$. The augmentation map will be denoted by $\varepsilon: B \rightarrow B_{0}=\tilde{R}$. More generally, the projection map to the $i$-th homogeneous component will be denoted by $\operatorname{pr}_{i}: B \rightarrow B_{i}$.

Example 2.2. (1) The ring of formal power series $R[[T]]$ is an $R$-cga with $i$-th homogeneous component $R \cdot T^{i}$.

(2) The ring $\tilde{R}$ is a cga with $(\tilde{R})_{0}=\tilde{R}$ and $(\tilde{R})_{i}=0$ for $i>0$. In particular, the ring $R$ itself is the trivial $R$-cga with $(R)_{i}=0$ for $i>0$.

Remark 2.3. (1) Similar to the notation of a power series as an infinite sum, elements of a cga $B$ are denoted by $\sum_{i=0}^{\infty} b_{i}$, where $b_{i} \in B_{i}$. This notation is also justified by the fact that, indeed, $\sum_{i=0}^{\infty} b_{i}$ is the limit of the sequence of partial sums $\left(\sum_{i=0}^{n} b_{i}\right)_{n \in \mathbb{N}}$ in the given topology, or in other words, $\sum_{i=0}^{\infty} b_{i}$ is a convergent series.

(2) Since $\bigoplus_{k=0}^{\infty} B_{k}$ is dense in $B$, the continuous extension of a given homomorphism of graded $R$-algebras is unique. By a homomorphism of cgas, we will always mean a homomorphism that is induced by a graded homomorphism of the underlying graded algebras.

${ }^{1}$ This separability condition is missing in MvdP03, but it has been added for example in Ama07, and Hei07. 
(3) For two cgas $B$ and $\tilde{B}$, we define the tensor product $B \otimes \tilde{B}$, namely the cga with homogeneous components $(B \otimes \tilde{B})_{k}:=\sum_{i+j=k} B_{i} \otimes_{R} \tilde{B}_{j}$.

We sometimes have to consider homomorphisms between cgas that aren't induced by homomorphisms of graded algebras. So let $B$ and $\tilde{B}$ be cgas and let $g: B \rightarrow \tilde{B}$ be a continuous homomorphism of $K$-modules (or even $K$-algebras). Then we define "homogeneous components" $g^{(i)}: B \rightarrow \tilde{B}(i \in \mathbb{Z})$ of $g$ to be the continuous homomorphisms of $K$-modules given by

$$
\left.g^{(i)}\right|_{B_{j}}:=\left.\operatorname{pr}_{i+j} \circ g\right|_{B_{j}}: B_{j} \rightarrow \tilde{B}_{i+j}
$$

for all $j \in \mathbb{N}\left(\right.$ set $\tilde{B}_{i+j}:=0$ for $\left.i+j<0\right)$. The $g^{(i)}$ uniquely determine $g$, because for all $b_{j} \in B_{j}, \sum_{i=-j}^{\infty} g^{(i)}\left(b_{j}\right)$ converges to $g\left(b_{j}\right)$.

Such a continuous homomorphism of $K$-modules $g: B \rightarrow \tilde{B}$ is called positive if $g^{(i)}=0$ for $i<0$, and we denote by $\operatorname{Hom}_{K}^{+}(B, \tilde{B})$ the set of positive continuous homomorphisms of $K$-modules from $B$ to $\tilde{B}$. A short calculation shows that for cgas $B$ and $\tilde{B}$, a continuous homomorphism $g: B \rightarrow \tilde{B}$ is a homomorphism of $K$-algebras if and only if the maps $g^{(k)}$ satisfy the property

$$
\forall k \in \mathbb{N}, \forall r, s \in B: \quad g^{(k)}(r s)=\sum_{i+j=k} g^{(i)}(r) g^{(j)}(s) .
$$

Furthermore, the monoid $(K, \cdot)$ acts on the set $\operatorname{Hom}_{K}^{+}(B, \tilde{B})$ of positive continuous homomorphisms of $K$-modules by

$$
(a . g)^{(i)}:=a^{i} \cdot g^{(i)} \quad(i \geq 0)
$$

for all $a \in K, g \in \operatorname{Hom}_{K}^{+}(B, \tilde{B})$. If $g$ is a homomorphism of algebras, then a.g is also a homomorphism of algebras. Moreover, for $g \in \operatorname{Hom}_{K}^{+}(B, \tilde{B}), h \in \operatorname{Hom}_{K}^{+}(\tilde{B}, \tilde{\tilde{B}})$ and $a \in K$, we have

$$
a .(h \circ g)=a . h \circ a . g,
$$

i. e. the action of $K$ commutes with composition.

Definition 2.4. For a cga $B$, a $\mathbf{c g m}$ over $B$ is the completion of a graded module over the graded algebra $\bigoplus_{k=0}^{\infty} B_{k}$, the completion taken by the topology induced from the grading. In the same manner as for cgas, we define homogeneous components of cgms, continuous homomorphisms between cgms and homogeneous components of those. There is also an action of the monoid $(K, \cdot)$ on the set of positive continuous homomorphisms between two given cgms.

Remark 2.5. Some special maps that we will use here are the higher derivations on rings and modules (cf. Sections 3 and 4) - maps in $\operatorname{Hom}_{K}^{+}(R, B)$, resp. $\operatorname{Hom}_{K}^{+}\left(M, B \otimes_{R} M\right)$-, the extension $\mathrm{d}_{\hat{\Omega}}$ of the universal derivation to the algebra of higher differentials - a map in $\operatorname{Hom}_{K}^{+}(\hat{\Omega}, \hat{\Omega})$ (cf. Section 3) -, the extensions of iterable higher derivations (cf. Section 10), as well as the extensions of higher connections on $M$ to maps in $\operatorname{Hom}_{K}^{+}\left(\hat{\Omega} \otimes_{R} M, \hat{\Omega} \otimes_{R} M\right)$ (cf. Section 4) and the extensions of iterable higher derivations on $M$ (cf. Section [10). 


\section{Higher DERIVATIONS AND HIGHER DIFFERENTIALS}

In this section we explain the notion of higher derivations on rings and modules. The definition used here is different from that introduced by Hasse and Schmidt in HS37. In fact, it is a generalisation, as we will show later on. This more general definition is necessary to define the algebra of higher differentials as a universal object.

Definition 3.1. Let $B$ be a $\tilde{R}$-cga. (As mentioned earlier $R, \tilde{R}$ denote integral domains over $K$ together with a homomorphism of $K$-algebras $f: R \rightarrow \tilde{R}$.) A higher derivation of $R$ to $B$ over $K$ is a homomorphism of $K$-algebras $\psi: R \rightarrow B$ satisfying $\varepsilon \circ \psi=f: R \rightarrow B_{0}=\tilde{R}$. The set of all higher derivations of $R$ to $B$ over $K$ will be denoted by $\operatorname{HD}_{K}(R, B)$. In the special case of $B=R[[T]]$ (and $\tilde{R}=R$, $\left.f=\mathrm{id}_{R}\right)$ we set $\operatorname{HD}_{K}(R):=\operatorname{HD}_{K}(R, R[[T]])$.

Remark 3.2. (1) Since a higher derivation $\psi \in \operatorname{HD}_{K}(R, B)$ can be regarded as a positive continuous homomorphism from the cga $R$ to the cga $B$, the "homogeneous components" of $\psi$ are denoted by $\psi^{(k)}: R \rightarrow B_{k}$, and for every $r \in R$, we then have $\psi(r)=\sum_{k=0}^{\infty} \psi^{(k)}(r)$. (The right hand side is a series that converges in the topology of $B$.)

(2) Let $\psi \in \operatorname{HD}_{K}(R)$. Then since $\varepsilon \circ \psi=\operatorname{id}_{R}$, the maps $\psi^{(k)}: R \rightarrow R \cdot T^{k} \cong R$ are homomorphisms of $K$-modules and satisfy the following properties:

$$
\begin{aligned}
\psi^{(0)} & =\operatorname{id}_{R}, \\
\forall k \in \mathbb{N}, \forall r, s \in R: \quad \psi^{(k)}(r s) & =\sum_{i+j=k} \psi^{(i)}(r) \psi^{(j)}(s) .
\end{aligned}
$$

Furthermore, any sequence $\left(\partial^{(k)}\right)_{k \in \mathbb{N}}$ of $K$-module-homomorphisms $\partial^{(k)}$ : $R \rightarrow R$ satisfying these two properties defines a higher derivation $\psi: R \rightarrow$ $R[[T]]$ via $\psi(r):=\sum_{k=0}^{\infty} \partial^{(k)}(r) T^{r}$.

(3) As mentioned in the beginning, Hasse and Schmidt defined a higher derivation to be a sequence $\left(\partial^{(k)}\right)_{k \in \mathbb{N}}$ as above. Hence our definition of a higher derivation $\psi \in \operatorname{HD}_{K}(R)$ is equivalent to that of Hasse and Schmidt.

Example 3.3. (1) If the characteristic of $K$ is zero, then any $K$-derivation $\partial: R \rightarrow R$ (in the classical sense) gives rise to a higher derivation $\phi_{\partial} \in$ $\mathrm{HD}_{K}(R)$ by

$$
\phi_{\partial}(r):=\sum_{k=0}^{\infty} \frac{1}{k !} \partial^{k}(r) T^{k}
$$

(see also Section 7).

(2) For a polynomial algebra $R=K\left[t_{1}, \ldots, t_{m}\right]$, every higher derivation of $R$ into some $R$-cga $B$ is given by an $m$-tuple $\left(b_{1}, \ldots, b_{m}\right)$ of elements of $B$ satisfying $\varepsilon\left(b_{j}\right)=t_{j}$ for all $j=1, \ldots, m$.

The higher derivations $\phi_{t_{j}} \in \mathrm{HD}_{K}\left(K\left[t_{1}, \ldots, t_{m}\right]\right)$ given by $\phi_{t_{j}}\left(t_{i}\right)=t_{i}$ for $i \neq j$ and $\phi_{t_{j}}\left(t_{j}\right)=t_{j}+T$ play an important role. In the classical context, $\phi_{t_{j}}^{(1)}$ is just the partial derivation with respect to $t_{j}$. We therefore call $\phi_{t_{j}}$ the higher derivation with respect to $t_{j}$. If $\tilde{R}$ is formally étale over $K\left[t_{1}, \ldots, t_{m}\right]$ (see Definition 3.4 and Example 3.5), then the $\phi_{t_{j}} \in \mathrm{HD}_{K}\left(K\left[t_{1}, \ldots, t_{m}\right]\right)$ uniquely extend to higher derivations on $\tilde{R}$ 
by Proposition 3.7. These derivations will also be referred to as higher derivations with respect to $t_{j}$ and will also be denoted by $\phi_{t_{j}}$.

Definition 3.4 (cf. Gro64, Def. 19.10.2). Let $S, \tilde{S}$ be rings and $g: S \rightarrow \tilde{S}$ be a homomorphism of rings. $\tilde{S}$ is called formally étale over $S$ if, for each surjective homomorphism of rings $h: T \rightarrow \tilde{T}$ with nilpotent kernel and all homomorphisms $v: S \rightarrow T$ and $\tilde{v}: \tilde{S} \rightarrow \tilde{T}$ satisfying $\tilde{v} \circ g=h \circ v$, there exists a unique homomorphism $u: \tilde{S} \rightarrow T$ such that $u \circ g=v$ and $h \circ u=\tilde{v}$, i. e., one obtains the following commutative diagram:

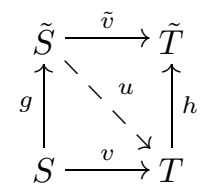

Example 3.5. (1) As it is shown in Gro64, Example 19.10.3(ii), localisations of a ring $S$ are formally étale over $S$.

(2) Every finite separable extension of a field $F$ is formally étale over $F$ (cf. Gro64, Ex. 19.10.3(iii)).

A more general example is the following:

Proposition 3.6. Let $S$ be a ring, and let $\tilde{S}=S(y)$ be an extension of $S$ with minimal polynomial $m(X) \in S[X]$ of $y$ and such that $m^{\prime}(y)$ is invertible in $\tilde{S}$, where $m^{\prime}(X):=\frac{d}{d X} m(X)$. Then $\tilde{S}$ is formally étale over $S$.

Proof. Let $h: T \rightarrow \tilde{T}$ be a surjective homomorphism with nilpotent kernel $I:=$ $\operatorname{Ker}(h)$ and let $v: S \rightarrow T$ and $\tilde{v}: \tilde{S} \rightarrow \tilde{T}$ be as in Definition 3.4. Since every lift $u$ of $v$ is given by the image of $y$ in $T$, we have to show that there exists a unique element $z \in T$ with $h(z)=v(y)=: \tilde{z}$ and $m(z)=0$. (By abuse of notation, we also write $m(X)$ for the polynomial $v(m)(X) \in T[X]$ and also for the polynomial $h(v(m))(X) \in \tilde{T}[X]$.) We will show by induction that for each $k \geq 1$ there exists a $z_{k} \in h^{-1}(\tilde{z})$ with $m\left(z_{k}\right) \in I^{k}$ and that $z_{k}$ is unique modulo $I^{k}$ with this property. Since $I$ is nilpotent, this proves the claim by choosing $k$ sufficiently large.

For $k=1$, any preimage $z_{1}$ of $\tilde{z}$ works, since $I=\operatorname{Ker}(h)$. Now assume that for given $k \geq 1$ there is a $z_{k} \in T$ satisfying $h\left(z_{k}\right)=\tilde{z}$ and $m\left(z_{k}\right) \in I^{k}$, which is unique modulo $I^{k}$. Since $m^{\prime}(y)$ is invertible in $\tilde{S}$, we have $m^{\prime}(\tilde{z}) \in \tilde{T}^{\times}$, and so $m^{\prime}\left(z_{k}\right) \in T^{\times}$, by Mats89, Ex. 1.1.

Using Taylor expansion, for $\zeta \in I^{k}$ we have $m\left(z_{k}+\zeta\right) \equiv m\left(z_{k}\right)+m^{\prime}\left(z_{k}\right) \zeta$ $\bmod I^{k+1}$. Therefore $m\left(z_{k}+\zeta\right) \in I^{k+1}$ if and only if $\zeta \equiv-m^{\prime}\left(z_{k}\right)^{-1} m\left(z_{k}\right)$ $\bmod I^{k+1}$. Since by hypothesis $m\left(z_{k}\right) \in I^{k}$ and hence $-m^{\prime}\left(z_{k}\right)^{-1} m\left(z_{k}\right) \in I^{k}$, the element $z_{k+1}:=z_{k}-m^{\prime}\left(z_{k}\right)^{-1} m\left(z_{k}\right) \in T$ satisfies $h\left(z_{k+1}\right)=\tilde{z}$ and $m\left(z_{k+1}\right) \in I^{k+1}$, and $z_{k+1}$ is unique modulo $I^{k+1}$ with these properties, since $z_{k}$ was unique modulo $I^{k}$.

We return to higher derivations (again using the notation given at the beginning of Section 21).

Proposition 3.7. If $\tilde{R}$ is formally étale over $R$, then every higher derivation $\psi \in$ $\operatorname{HD}_{K}(R, B)$ to a $\tilde{R}$-cga $B$ can be uniquely extended to a higher derivation $\psi_{e} \in$ $\operatorname{HD}_{K}(\tilde{R}, B)$. 
The proof is almost identical to H. Matsumura's proof for the case $B=\tilde{R}[[T]]$, so we will omit it here. (See [Mats89], Thm. 27.2; formally étale is called 0-etale there.)

Definition 3.8. For $\psi \in \operatorname{HD}_{K}(R)$ we define a continuous endomorphism $\psi[[T]]$ on $R[[T]]$ by $\psi[[T]]\left(\sum_{i=0}^{\infty} a_{i} T^{i}\right):=\sum_{i=0}^{\infty} \psi\left(a_{i}\right) T^{i}$. (In fact, $\psi[[T]]$ is an automorphism.) Using this we get a multiplication on $\operatorname{HD}_{K}(R)$ by

$$
\psi_{1} \cdot \psi_{2}:=\psi_{1}[[T]] \circ \psi_{2} \in \operatorname{HD}_{K}(R)
$$

for $\psi_{1}, \psi_{2} \in \operatorname{HD}_{K}(R)$. This defines a group structure on $\operatorname{HD}_{K}(R)$ (see Mats89, $\S 27)$.

The link to (ordinary) derivations is given by

Proposition 3.9. For $\operatorname{char}(K)=0$, the set $\operatorname{Der}(R):=\left\{\psi^{(1)} \mid \psi \in \operatorname{HD}_{K}(R)\right\}$ is the $R$-module of derivations on $R$ (cf. Proposition 7.1 ).

We now turn our attention to the universal higher derivation:

Theorem 3.10. Up to isomorphism, there exists a unique $R$-cga $\hat{\Omega}_{R / K}$ (which we call the algebra of higher differentials), together with a higher derivation $\mathrm{d}_{R}: R \rightarrow \hat{\Omega}_{R / K}$ satisfying the following universal property:

For each $\tilde{R}$-cga $B$ and each higher derivation $\psi: R \rightarrow B$, there exists a unique homomorphism of $\tilde{R}$-cgas $\tilde{\psi}: \tilde{R} \otimes \hat{\Omega}_{R / K} \rightarrow B$ with $\tilde{\psi} \circ\left(1 \otimes \mathrm{d}_{R}\right)=\psi$. In other words, $\hat{\Omega}_{R / K}$ represents the functor $\operatorname{HD}_{K}(R,-)$.

Proof. We construct $\hat{\Omega}_{R / K}$. Uniqueness is given by the universal property.

Let $G=R\left[\mathrm{~d}^{(k)} r \mid k \in \mathbb{N}_{+}, r \in R\right]$ be the polynomial algebra over $R$ in the variables $\mathrm{d}^{(k)} r$ and let the degree of $\mathrm{d}^{(k)} r$ be $k$. Define $I \unlhd G$ to be the ideal generated by the union of the sets

$$
\begin{aligned}
& \left\{\mathrm{d}^{(k)}(r+s)-\mathrm{d}^{(k)} r-\mathrm{d}^{(k)} s \mid k \in \mathbb{N}_{+} ; r, s \in R\right\}, \\
& \left\{\mathrm{d}^{(k)} a \mid k \in \mathbb{N}_{+} ; a \in K\right\} \quad \text { and } \\
& \left\{\mathrm{d}^{(k)}(r s)-\sum_{i=0}^{k} \mathrm{~d}^{(i)} r \cdot \mathrm{d}^{(k-i)} s \mid k \in \mathbb{N}_{+} ; r, s \in R\right\},
\end{aligned}
$$

where we set $\mathrm{d}^{(0)} r=r$ for all $r \in R$. Therefore $I$ is a homogeneous ideal, and we define $\hat{\Omega}_{R / K}$ to be the completion of the graded algebra $G / I$. We also define the higher derivation $\mathrm{d}_{R}: R \rightarrow \hat{\Omega}_{R / K}$ by $\mathrm{d}_{R}(r):=\sum_{k=0}^{\infty} \mathrm{d}^{(k)} r$. (Here and in the following the residue class of $\mathrm{d}^{(k)} r \in G$ in $\hat{\Omega}_{R / K}$ will also be denoted by $\mathrm{d}^{(k)} r$.)

The universal property is seen as follows: Let $\psi: R \rightarrow B$ be a higher derivation. Then we define an $R$-algebra-homomorphism $g: G \rightarrow B$ by $g\left(\mathrm{~d}^{(k)} r\right):=\psi^{(k)}(r)$ for all $k>0$ and $r \in R$. The properties of a higher derivation imply that $I$ lies in the kernel of $g$, and therefore $g$ factors through $\bar{g}: G / I \rightarrow B$. Hence, we get a homomorphism of algebras $\hat{\Omega}_{R / K} \rightarrow B$ by extending $\bar{g}$ continuously and therefore a homomorphism of $\tilde{R}$-cgas $\tilde{\psi}: \tilde{R} \otimes \hat{\Omega}_{R / K} \rightarrow B$. On the other hand, the condition $\tilde{\psi} \circ\left(1 \otimes \mathrm{d}_{R}\right)=\psi$ forces this choice of $g$, and so $\tilde{\psi}$ is unique.

Remark 3.11. In Voj07, P. Vojta defined an $R$-algebra $H S_{R / K}^{\infty}$ that represents the functor $\tilde{R} \mapsto \operatorname{HD}_{K}(R, \tilde{R}[[T]])$ for any $R$-algebra $\tilde{R}$. Actually, $H S_{R / K}^{\infty}$ is a 
graded algebra, and by construction $\hat{\Omega}_{R / K}$ is just the completion of $H S_{R / K}^{\infty}$ (cf. the construction of $H S_{R / K}^{\infty}$ in Voj07, Def. 1.3). Hence, some properties of $\hat{\Omega}_{R / K}$ can be deduced from the properties of $H S_{R / K}^{\infty}$ given in Voj07.

Proposition 3.12. $\quad$ (a) For every homomorphism of rings $f: R \rightarrow \tilde{R}$ there is a unique homomorphism of $\tilde{R}$-cgas $D f: \tilde{R} \otimes \hat{\Omega}_{R / K} \rightarrow \hat{\Omega}_{\tilde{R} / K}$ such that $\mathrm{d}_{\tilde{R}} \circ f=D f \circ\left(1 \otimes \mathrm{d}_{R}\right)$.

(b) If $\tilde{R}$ is formally étale over $R$, then $D f$ is an isomorphism.

Proof. Since $\mathrm{d}_{\tilde{R}} \circ f$ is a higher derivation on $R$, part (a) follows from the universal property of $\hat{\Omega}_{R / K}$. Part (b) follows from Voj07, Thm. 3.6, where it is shown that the homomorphism of the underlying graded algebras is an isomorphism in this case.

We consider three important examples.

Theorem 3.13. $\quad$ (a) Let $R=K\left[t_{1}, \ldots, t_{m}\right]$ be the polynomial ring in $m$ variables. Then $\hat{\Omega}_{R / K}$ is the completion of the polynomial algebra $R\left[\mathrm{~d}^{(i)} t_{j} \mid i \in \mathbb{N}_{+}, j=1, \ldots, m\right]$.

(b) Let $F / K\left(t_{1}, \ldots, t_{m}\right)$ be a finite separable field extension. Then $\hat{\Omega}_{F / K}$ is the completion of the polynomial algebra $F\left[\mathrm{~d}^{(i)} t_{j} \mid i \in \mathbb{N}_{+}, j=1, \ldots, m\right]$.

(c) Let $(R, \mathfrak{m})$ be a regular local ring of dimension $m$, let $t_{1}, \ldots, t_{m}$ generate $\mathfrak{m}$ and assume that $R$ is a localisation of a finitely generated $K$-algebra and that $R / \mathfrak{m}$ is a finite separable extension of $K$. Then $\hat{\Omega}_{R / K}$ is the completion of the polynomial algebra $R\left[\mathrm{~d}^{(i)} t_{j} \mid i \in \mathbb{N}_{+}, j=1, \ldots, m\right]$.

Remark 3.14. The completion of such a polynomial algebra will be denoted by $R\left[\left[\mathrm{~d}^{(i)} t_{j} \mid i \in \mathbb{N}_{+}, j=1, \ldots, m\right]\right]$, although it is not really a ring of power series, because it contains infinite sums of different variables.

Proof. Part (a) is a direct consequence of Voj07, Prop. 5.1. Part (b) then follows from Proposition 3.12(b), since by Example $3.5 K\left(t_{1}, \ldots, t_{m}\right)$ is formally étale over $K\left[t_{1}, \ldots, t_{m}\right]$ and $F$ is formally étale over $K\left(t_{1}, \ldots, t_{m}\right)$.

It remains to prove (c): We will show that $(R / \mathfrak{m}) \otimes \hat{\Omega}_{R / K}$ is isomorphic to $(R / \mathfrak{m})\left[\left[\mathrm{d}^{(i)} t_{j}\right]\right]$. Then, since $\operatorname{Quot}(R) \otimes \hat{\Omega}_{R / K}$ is isomorphic to $\operatorname{Quot}(R)\left[\left[\mathrm{d}^{(i)} t_{j}\right]\right]$ (Proposition 3.12 and part (b)), by Hart77, Ch. II, Lemma 8.9, it follows that $\left(\hat{\Omega}_{R / K}\right)_{k}$ is a free $R$-module and that the residue classes of any basis of $\left(\hat{\Omega}_{R / K}\right)_{k}$ form a basis of $\left(R / \mathfrak{m} \otimes \hat{\Omega}_{R / K}\right)_{k}$. Hence we obtain $\hat{\Omega}_{R / K}=R\left[\left[\mathrm{~d}^{(i)} t_{j}\right]\right]$.

First, let $\psi: R \rightarrow B$ be a higher derivation of $R$ to an $R / \mathfrak{m}$-cga $B$. Then for all $k \in \mathbb{N}$ and $r_{1}, \ldots, r_{k+1} \in \mathfrak{m}$, we have

$$
\psi^{(k)}\left(r_{1} \cdots r_{k+1}\right)=\sum_{i_{1}+\cdots+i_{k+1}=k} \psi^{\left(i_{1}\right)}\left(r_{1}\right) \cdots \psi^{\left(i_{k+1}\right)}\left(r_{k+1}\right)=0,
$$

since in each summand at least one $i_{j}=0$, and so $\psi^{\left(i_{1}\right)}\left(r_{1}\right) \cdots \psi^{\left(i_{k+1}\right)}\left(r_{k+1}\right) \in$ $\mathfrak{m} B=0$. Therefore $\psi^{(k)}$ (and $\psi^{(i)}$ for $i<k$ ) factors through $R / \mathfrak{m}^{k+1}$.

Next, since $R / \mathfrak{m}$ is a finite separable extension of $K$, there is an element $\bar{y} \in$ $R / \mathfrak{m}$ which generates the extension $K \subset R / \mathfrak{m}$. Let $g(X) \in K[X]$ be the minimal polynomial of $\bar{y}$. Then starting with an arbitrary representative $y \in R$ for $\bar{y}$, using the Newton approximation $y_{n+1}=y_{n}-g\left(y_{n}\right) g^{\prime}\left(y_{n}\right)^{-1}$, we obtain an element 
$\tilde{y}_{k} \in R$ such that $g\left(\tilde{y}_{k}\right) \equiv 0 \bmod \mathfrak{m}^{k+1}$ for given $k \in \mathbb{N}$. (Note that the Newton approximation is well defined and converges to a root of $g(X)$, due to the fact that $\overline{g(y)}=g(\bar{y})=0 \in R / \mathfrak{m}$ and $\overline{g^{\prime}(y)}=g^{\prime}(\bar{y}) \neq 0 \in R / \mathfrak{m}$, so $g(y) \in \mathfrak{m}$ and $g(y) \in R^{\times}$, as well as inductively for all $n \in \mathbb{N}: \bar{y}_{n+1}=\bar{y}_{n}=\bar{y} \in R / \mathfrak{m}, g\left(y_{n+1}\right) \in \mathfrak{m}$ and $g^{\prime}\left(y_{n+1}\right) \in R^{\times}$.) This proves that for all $k \in \mathbb{N}$, the ring $R / \mathfrak{m}^{k+1}$ contains a subfield isomorphic to $R / \mathfrak{m}$.

Now by Mats89, Theorem 14.4, the associated graded ring $\operatorname{gr}(R)$ of $R$ is isomorphic to the polynomial ring $(R / \mathfrak{m})\left[t_{1}, \ldots, t_{m}\right]$, and therefore we obtain $\operatorname{gr}\left(R / \mathfrak{m}^{k+1}\right)$ $\cong(R / \mathfrak{m})\left[t_{1}, \ldots, t_{m}\right] / \mathfrak{n}^{k+1}$, where $\mathfrak{n}$ is the ideal generated by $\left\{t_{1}, \ldots, t_{m}\right\}$. Furthermore, since $R / \mathfrak{m}^{k+1}$ contains a subfield isomorphic to $R / \mathfrak{m}$, we see that the inclusion $\iota_{k}:(R / \mathfrak{m})\left[t_{1}, \ldots, t_{m}\right] / \mathfrak{n}^{k+1} \rightarrow R / \mathfrak{m}^{k+1}$ (given by the inclusion $K\left[t_{1}, \ldots, t_{m}\right] / \mathfrak{n}^{k+1}$ $\subset R / \mathfrak{m}^{k+1}$ and $\left.\bar{y} \mapsto \tilde{y}_{k}\right)$ is an isomorphism.

Hence, every higher derivation $\psi_{\mathrm{gr}}: \operatorname{gr}(R) \rightarrow B$ into an $R / \mathfrak{m}$-cga $B$ induces a higher derivation $\psi_{R}: R \rightarrow B$ on $R$ by $\psi_{R}^{(k)}:=\psi_{\mathrm{gr}}^{(k)} \circ \iota_{k}^{-1}(k \in \mathbb{N})$, and vice versa. So $R / \mathfrak{m} \otimes_{R} \hat{\Omega}_{R / K} \cong R / \mathfrak{m} \otimes_{\operatorname{gr}(R)} \hat{\Omega}_{\operatorname{gr}(R) / K}=(R / \mathfrak{m})\left[\left[\mathrm{d}^{(i)} t_{j}\right]\right]$.

Corollary 3.15. Let $R$ be a finitely generated $K$-algebra which is a regular ring. Then the homogeneous components $\left(\hat{\Omega}_{R / K}\right)_{k}(k \in \mathbb{N})$ are projective $R$-modules of finite rank.

Proof. For every maximal ideal $\mathfrak{m} \unlhd R$, the localisation $R_{\mathfrak{m}}$ fulfills the conditions of Theorem 3.13(c). Therefore, by Proposition 3.12, $R_{\mathfrak{m}} \otimes_{R}\left(\hat{\Omega}_{R / K}\right)_{k} \cong\left(\hat{\Omega}_{R_{\mathfrak{m}} / K}\right)_{k}$ is a free $R_{\mathfrak{m}}$-module of finite rank. Hence by [Eis95], Thm. A3.2, $\left(\hat{\Omega}_{R / K}\right)_{k}$ is a projective $R$-module of finite rank.

From now on we also write $\hat{\Omega}$ for $\hat{\Omega}_{R / K}$.

Theorem 3.16. For each $a \in K$, there is a continuous homomorphism of $K$ algebras $a . \mathrm{d}_{\hat{\Omega}}: \hat{\Omega} \rightarrow \hat{\Omega}$ defined by

$$
a \cdot \mathrm{d}_{\hat{\Omega}}\left(\mathrm{d}_{R}^{(i)} r\right):=\sum_{j=0}^{\infty} a^{j}\left(\begin{array}{c}
i+j \\
j
\end{array}\right) \mathrm{d}_{R}^{(i+j)} r
$$

for all $i \in \mathbb{N}$ and $r \in R$. The homomorphisms a. $\mathrm{d}_{\hat{\Omega}}$ satisfy the following three conditions:

(1) $a . \mathrm{d}_{\hat{\Omega}}$ extends the higher derivation $a . \mathrm{d}_{R}: R \rightarrow \hat{\Omega}$.

(2) For all $a, b \in K,\left(a \cdot \mathrm{d}_{\hat{\Omega}}\right) \circ\left(b \cdot \mathrm{d}_{\hat{\Omega}}\right)=(a+b) \cdot \mathrm{d}_{\hat{\Omega}}$.

(3) $0 . \mathrm{d}_{\hat{\Omega}}=\mathrm{id}_{\hat{\Omega}}$.

For short, we will write $\mathrm{d}_{\hat{\Omega}}$ instead of $1 . \mathrm{d}_{\hat{\Omega}}$ and $-\mathrm{d}_{\hat{\Omega}}$ instead of $-1 . \mathrm{d}_{\hat{\Omega}}$.

Proof. It is not hard to check that $a . \mathrm{d}_{\hat{\Omega}}$ is well defined. Then the first and third statements are obvious, and the second one is shown by an explicit calculation using some combinatorial identities (see [Rös07], Theorem 2.5, for details).

Remark 3.17. (1) By the second and the third properties we see that $a . \mathrm{d}_{\hat{\Omega}}$ is actually an automorphism of $\hat{\Omega}$ for all $a \in K$. The endomorphisms $a . \mathrm{d}_{\hat{\Omega}}$ play an important role in the iterative theory, as will be seen in Section 5

(2) From the definition, we see that $a . \mathrm{d}_{\hat{\Omega}}$ is the image of $\mathrm{d}_{\hat{\Omega}}$ under the action of $a \in K$, as given in Section 2, thus the notation $a . \mathrm{d}_{\hat{\Omega}}$. 
Proposition 3.18. For all $i, j \in \mathbb{N}$ we have

$$
\mathrm{d}_{\hat{\Omega}}^{(i)} \circ \mathrm{d}_{\hat{\Omega}}^{(j)}=\left(\begin{array}{c}
i+j \\
i
\end{array}\right) \mathrm{d}_{\hat{\Omega}}^{(i+j)}
$$

where $\mathrm{d}_{\hat{\Omega}}^{(i)}$ denotes the $i$-th homogeneous component of $\mathrm{d}_{\hat{\Omega}}$ (cf. Section 2).

Proof. For all $i, j \in \mathbb{N}$ and $\omega \in \hat{\Omega}$, the term $\left(\mathrm{d}_{\hat{\Omega}}^{(i)} \circ \mathrm{d}_{\hat{\Omega}}^{(j)}\right)(\omega)$ is the coefficient of $a^{i} b^{j}$ in the expression $\left(\left(a . \mathrm{d}_{\hat{\Omega}}\right) \circ\left(b . \mathrm{d}_{\hat{\Omega}}\right)\right)(\omega)$. Furthermore by Theorem 3.16, $\left(a \cdot \mathrm{d}_{\hat{\Omega}}\right) \circ\left(b \cdot \mathrm{d}_{\hat{\Omega}}\right)=(a+b) \cdot \mathrm{d}_{\hat{\Omega}}$, and so $\left(\mathrm{d}_{\hat{\Omega}}^{(i)} \circ \mathrm{d}_{\hat{\Omega}}^{(j)}\right)(\omega)$ is the coefficient of $a^{i} b^{j}$ in the expression $(a+b) \cdot \mathrm{d}_{\hat{\Omega}}(\omega)=\sum_{k=0}^{\infty}(a+b)^{k} \mathrm{~d}_{\hat{\Omega}}^{(k)}(\omega)$, i. e., equals $\left(\begin{array}{c}i+j \\ i\end{array}\right) \mathrm{d}_{\hat{\Omega}}^{(i+j)}(\omega)$. (For a finite field $K$, one has to use the little trick explained in Remark 5.6 to justify this conclusion.)

\section{Higher DERIVATIONS ON MOdULES AND HIGHER CONNECTIONS}

In the following, $M$ will denote a finitely generated $R$-module and $B$ will be a $\tilde{R}$-cga.

Definition 4.1. Let $\psi: R \rightarrow B$ be a higher derivation of $R$ to $B$ over $K$. A (higher) $\psi$-derivation of $M$ is an additive map $\Psi: M \rightarrow B \otimes_{R} M$ with $(\varepsilon \otimes$ $\left.\operatorname{id}_{M}\right) \circ \Psi=f \otimes \operatorname{id}_{M}$ and $\Psi(r m)=\psi(r) \Psi(m)$ for all $r \in R, m \in M$. The set of $\psi$-derivations of $M$ is denoted by $\operatorname{HD}(M, \psi)$.

A higher connection on $M$ is a $\mathrm{d}_{R}$-derivation $\nabla \in \operatorname{HD}\left(M, \mathrm{~d}_{R}\right)$. If $\nabla$ is a higher connection on $M$, for any higher derivation $\psi \in \operatorname{HD}_{K}(R, B)$ we define the $\psi$-derivation $\nabla_{\psi}$ on $M$ by

$$
\nabla_{\psi}:=\left(\tilde{\psi} \otimes \operatorname{id}_{M}\right) \circ \nabla: M \rightarrow \hat{\Omega}_{R / K} \otimes_{R} M \rightarrow B \otimes_{R} M
$$

(cf. Remark $4.2(\mathrm{i})$ ).

For all $a \in K$ we define an endomorphism $a_{\cdot_{\hat{\Omega}}} \nabla: \hat{\Omega} \otimes_{R} M \rightarrow \hat{\Omega} \otimes_{R} M$ by

$$
(a \cdot \hat{\Omega} \nabla)(\omega \otimes x):=a \cdot \mathrm{d}_{\hat{\Omega}}(\omega) \cdot(a . \nabla)(x)
$$

for all $\omega \in \hat{\Omega}$ and $x \in M$, i.e. $a \cdot{ }_{\hat{\Omega}} \nabla=\left(\mu_{\hat{\Omega}} \otimes \operatorname{id}_{M}\right) \circ\left(a \cdot \mathrm{d}_{\hat{\Omega}} \otimes a . \nabla\right)$, where $\mu_{\hat{\Omega}}$ denotes the multiplication map in $\hat{\Omega}$.

Remark 4.2. (i) For a given $\psi \in \operatorname{HD}_{K}(R, B)$, every homomorphism of $\tilde{R}$-cgas $g: B \rightarrow \tilde{B}$ induces a map $g_{*}: \operatorname{HD}(M, \psi) \rightarrow \operatorname{HD}(M, g \circ \psi), \Psi \mapsto\left(g \otimes \operatorname{id}_{M}\right) \circ \Psi$. Using the action of $K$ in this context, for every $\Psi \in \operatorname{HD}(M, \psi)$ and $a \in K$, we get an $(a . \psi)$-derivation $a . \Psi$.

(ii) Let $\psi_{1}, \psi_{2} \in \operatorname{HD}_{K}(R)$ and $\Psi_{i} \in \operatorname{HD}\left(M, \psi_{i}\right)(i=1,2)$. Then as in Definition 3.8 we have an automorphism $\Psi[[T]]$ of $R[[T]] \otimes M \cong M[[T]]$ and a product $\Psi_{1} \cdot \Psi_{2}$, which is an element of $\operatorname{HD}\left(M, \psi_{1} \psi_{2}\right)$.

Our next aim is to show that for a regular ring $R$ over a perfect field $K$ every $R$-module that admits a higher connection is projective (or, in geometric terms, locally free). So we get the analogue of the well-known fact in characteristic zero that a coherent sheaf equipped with a holomorphic connection must be locally free, resp. the analogue of the corresponding fact in the not necessarily commutative situation given by Y. André in And01, Cor. 2.5.2.2. 
We first need the following lemma:

Lemma 4.3. Let $(R, \mathfrak{m})$ be a regular local ring of dimension $m$, let $t_{1}, \ldots, t_{m}$ generate $\mathfrak{m}$ and assume that $R$ is a localisation of a finitely generated $K$-algebra and that $R / \mathfrak{m}$ is a finite separable extension of $K$. Let $\phi_{t_{j}} \in \operatorname{HD}_{K}(R)(j=1, \ldots, m)$ denote the higher derivations with respect to $t_{j}$ (cf. Example 3.3 and Thm. 3.13(c)).

Then for every $r \in R \backslash\{0\}$ there exist $k_{1}, \ldots, k_{m} \in \mathbb{N}$ such that

(1) $\left(\phi_{t_{m}}^{\left(k_{m}\right)} \circ \cdots \circ \phi_{t_{1}}^{\left(k_{1}\right)}\right)(r) \in R^{\times}$,

(2) for all $l_{1}, \ldots, l_{m} \in \mathbb{N}$ with $l_{j} \leq k_{j}(j=1, \ldots, m)$ and $l_{i}<k_{i}$ for some $i \in\{1, \ldots, m\}$,

$$
\left(\phi_{t_{m}}^{\left(l_{m}\right)} \circ \cdots \circ \phi_{t_{1}}^{\left(l_{1}\right)}\right)(r) \notin R^{\times} .
$$

Proof. Let $r \in R \backslash\{0\}$. Choose $E \in \mathbb{N}$ such that $r \in \mathfrak{m}^{E}$ and $r \notin \mathfrak{m}^{E+1}$. Then $r$ can (uniquely) be written as

$$
r=\sum_{\substack{\boldsymbol{e}=\left(e_{1}, \ldots, e_{m}\right) \in \mathbb{N}^{m} \\|e|=E}} u_{e} t^{e}
$$

where $u_{\boldsymbol{e}} \in R$ and $u_{\boldsymbol{f}} \in R^{\times}$for at least one $\boldsymbol{f}=\left(f_{1}, \ldots, f_{m}\right)$.

(We use the usual notation of multi-indices: $|\boldsymbol{e}|=e_{1}+\cdots+e_{m}$ and $\boldsymbol{t}^{\boldsymbol{e}}=$ $\left.t_{1}^{e_{1}} \cdots t_{m}^{e_{m}}.\right)$ For arbitrary $\boldsymbol{l}=\left(l_{1}, \ldots, l_{m}\right) \in \mathbb{N}^{m}$ and $\boldsymbol{e} \in \mathbb{N}^{m}$ we have

$$
\left(\phi_{t_{m}}^{\left(l_{m}\right)} \circ \cdots \circ \phi_{t_{1}}^{\left(l_{1}\right)}\right)\left(\boldsymbol{t}^{\boldsymbol{e}}\right)=\left(\begin{array}{l}
e_{1} \\
l_{1}
\end{array}\right) \cdots\left(\begin{array}{l}
e_{m} \\
l_{m}
\end{array}\right) \boldsymbol{t}^{\boldsymbol{e}-\boldsymbol{l}}=\left\{\begin{array}{cc}
0 & \text { if } l_{i}>e_{i} \text { for some } i \\
1 & \text { if } l_{j}=e_{j} \text { for all } j \\
\in \mathfrak{m} & \text { if }|\boldsymbol{l}|<|\boldsymbol{e}| .
\end{array}\right.
$$

So if we choose $k_{j}=f_{j}(j=1, \ldots, m)$, we get

$$
\begin{aligned}
& \left(\phi_{t_{m}}^{\left(k_{m}\right)} \circ \cdots \circ \phi_{t_{1}}^{\left(k_{1}\right)}\right)(r)=\sum_{|\boldsymbol{e}|=E}\left(\phi_{t_{m}}^{\left(k_{m}\right)} \circ \cdots \circ \phi_{t_{1}}^{\left(k_{1}\right)}\right)\left(u_{\boldsymbol{e}} \boldsymbol{t}^{\boldsymbol{e}}\right) \\
& \quad=\sum_{|\boldsymbol{e}|=E} \sum_{\substack{0 \leq l_{j} \leq k_{j} \\
j=1, \ldots, m}}\left(\phi_{t_{m}}^{\left(k_{m}-l_{m}\right)} \circ \cdots \circ \phi_{t_{1}}^{\left(k_{1}-l_{1}\right)}\right)\left(u_{\boldsymbol{e}}\right)\left(\phi_{t_{m}}^{\left(l_{m}\right)} \circ \cdots \circ \phi_{t_{1}}^{\left(l_{1}\right)}\right)\left(\boldsymbol{t}^{\boldsymbol{e}}\right) \\
& \equiv u_{\boldsymbol{f}} \cdot 1 \quad \bmod \mathfrak{m} .
\end{aligned}
$$

So $\left(\phi_{t_{m}}^{\left(k_{m}\right)} \circ \cdots \circ \phi_{t_{1}}^{\left(k_{1}\right)}\right)(r) \in u_{\boldsymbol{f}}+\mathfrak{m} \subset R^{\times}$, and for all $\boldsymbol{l} \in \mathbb{N}^{m}$ with $l_{j} \leq k_{j}$ $(j=1, \ldots, m)$ and $l_{i}<k_{i}$ for some $i$, we have $\left(\phi_{t_{m}}^{\left(l_{m}\right)} \circ \cdots \circ \phi_{t_{1}}^{\left(l_{1}\right)}\right)(r) \in \mathfrak{m}=R \backslash R^{\times}$, since $|\boldsymbol{l}|<E$.

Theorem 4.4. Let $(R, \mathfrak{m})$ be a regular local ring as in Lemma 4.3 and let $M$ be a finitely generated $R$-module with a higher connection $\nabla \in \operatorname{HD}(M, \mathrm{~d})$. Then $M$ is a free $R$-module.

Proof. Let $\left\{x_{1}, \ldots, x_{n}\right\}$ be a minimal set of generators of $M$. Assume that $x_{1}, \ldots, x_{n}$ are linearly dependent. Then there exists a nontrivial relation $\sum_{i=1}^{n} r_{i} x_{i}=0$, with $r_{i} \in R$. Choose $E \in \mathbb{N}$ such that $r_{j} \in \mathfrak{m}^{E}$ for all $j=1, \ldots n$ and $r_{i} \notin \mathfrak{m}^{E+1}$ for at 
least one $i$. Without loss of generality, let $r_{1} \notin \mathfrak{m}^{E+1}$. Then choose $k_{1}, \ldots, k_{m} \in \mathbb{N}$ for $r_{1}$ as given by the previous lemma. Then

$$
\begin{aligned}
0 & =\left(\nabla_{\phi_{t_{m}}}^{\left(k_{m}\right)} \circ \cdots \circ \nabla_{\phi_{t_{1}}}^{\left(k_{1}\right)}\right)\left(\sum_{i=1}^{n} r_{i} x_{i}\right) \\
& =\sum_{i=1}^{n} \sum_{\substack{0 \leq l_{j} \leq k_{j} \\
j=1, \ldots, m}}\left(\phi_{t_{m}}^{\left(l_{m}\right)} \circ \cdots \circ \phi_{t_{1}}^{\left(l_{1}\right)}\right)\left(r_{i}\right)\left(\nabla_{\phi_{t_{m}}}^{\left(k_{m}-l_{m}\right)} \circ \cdots \circ \nabla_{\phi_{t_{1}}}^{\left(k_{1}-l_{1}\right)}\right)\left(x_{i}\right) \\
& \equiv \sum_{i=1}^{n}\left(\phi_{t_{m}}^{\left(k_{m}\right)} \circ \cdots \circ \phi_{t_{1}}^{\left(k_{1}\right)}\right)\left(r_{i}\right) \cdot x_{i} \bmod \mathfrak{m} M .
\end{aligned}
$$

Since $\left(\phi_{t_{m}}^{\left(k_{m}\right)} \circ \cdots \circ \phi_{t_{1}}^{\left(k_{1}\right)}\right)\left(r_{1}\right) \in R^{\times}$, we get $x_{1} \in\left\langle x_{2}, \ldots, x_{n}\right\rangle+\mathfrak{m} M$, so $M=$ $\left\langle x_{2}, \ldots, x_{n}\right\rangle+\mathfrak{m} M$, and therefore by Nakayama's lemma $M=\left\langle x_{2}, \ldots, x_{n}\right\rangle$, in contradiction to the minimality of $\left\{x_{1}, \ldots, x_{n}\right\}$. So $x_{1}, \ldots, x_{n}$ is a basis for $M$, and in particular $M$ is a free $R$-module.

Corollary 4.5. Let $K$ be a perfect field and let $R$ be a finitely generated $K$-algebra which is a regular ring. Then every finitely generated $R$-module $M$ with higher connection $\nabla$ is a projective $R$-module.

Proof. By the previous theorem, the localisations of $M$ at every maximal ideal of $R$ are free. Hence $M$ is projective.

\section{ITERATIVE DERIVATIONS AND ITERATIVE CONNECTIONS}

Definition 5.1. A higher derivation $\phi \in \operatorname{HD}_{K}(R)$ is called an iterative derivation if

$$
\forall i, j \in \mathbb{N}: \quad \phi^{(i)} \circ \phi^{(j)}=\left(\begin{array}{c}
i+j \\
i
\end{array}\right) \phi^{(i+j)} .
$$

The set of iterative derivations on $R$ is denoted by $\operatorname{ID}_{K}(R)$.

Let $M$ be an $R$-module and $\phi \in \operatorname{ID}_{K}(R)$. A higher $\phi$-derivation $\Phi \in \operatorname{HD}(M, \phi)$ is called an iterative $\phi$-derivation if

$$
\forall i, j \in \mathbb{N}: \quad \Phi^{(i)} \circ \Phi^{(j)}=\left(\begin{array}{c}
i+j \\
i
\end{array}\right) \Phi^{(i+j)} .
$$

The set of iterative $\phi$-derivations is denoted by $\operatorname{ID}(M, \phi)$.

Example 5.2. If $R$ is the polynomial ring $K\left[t_{1}, \ldots, t_{m}\right]$ or a formally étale extension of that ring, the higher derivations $\phi_{t_{j}}$ with respect to $t_{j}$ (cf. Example 3.3) are iterative derivations. (For $K\left[t_{1}, \ldots, t_{m}\right]$ this is obvious, and for extensions, it follows from Lemma 5.8.)

Remark 5.3. Note that there is no sense in defining an iterative derivation $\Phi \in$ $\operatorname{HD}(M, \psi)$ for a noniterative higher derivation $\psi \in \operatorname{HD}_{K}(R)$. This is seen by using the characterisation of the iterative derivations in Lemma 5.4 and Lemma 5.7 For all $a, b \in K^{\mathrm{sep}},(a . \Phi)(b . \Phi)$ is an $(a . \psi)(b . \psi)$-derivation, whereas $(a+b) . \Phi$ is an $(a+b) \cdot \psi$-derivation. 
Lemma 5.4 (Characterisation of iterative derivations). Let $\psi \in \operatorname{HD}_{K}(R)$ be $a$ higher derivation. Then the following conditions are equivalent:

(i) $\psi$ is iterative,

(ii) $\tilde{\psi} \circ \mathrm{d}_{\hat{\Omega}}=\psi[[T]] \circ \tilde{\psi} \quad$ (see Theorem 3.10 for the definition of $\tilde{\psi}$ ),

(iii) For all $a \in K, \tilde{\psi} \circ\left(a . \mathrm{d}_{\hat{\Omega}}\right)=(a . \psi[[T]]) \circ \tilde{\psi}$.

If $K$ is an infinite field, then this is also equivalent to

(iv) For all $a, b \in K,(a . \psi)(b . \psi)=(a+b) . \psi$,

whereas for arbitrary $K$ the conditions (i)-(iii) only imply condition (iv).

Proof. For $a \in K, r \in R$ and $i \in \mathbb{N}$ we have

$$
\tilde{\psi} \circ\left(a . \mathrm{d}_{\hat{\Omega}}\right)\left(\mathrm{d}^{(i)} r\right)=\tilde{\psi}\left(\sum_{j=0}^{\infty} a^{j}\left(\begin{array}{c}
i+j \\
j
\end{array}\right) \mathrm{d}^{(i+j)} r\right)=\sum_{j=0}^{\infty} a^{j}\left(\begin{array}{c}
i+j \\
j
\end{array}\right) \psi^{(i+j)}(r) T^{i+j}
$$

and

$$
(a . \psi[[T]]) \circ \tilde{\psi}\left(\mathrm{d}^{(i)} r\right)=a . \psi[[T]]\left(\psi^{(i)}(r) T^{i}\right)=\sum_{j=0}^{\infty} a^{j} \psi^{(j)}\left(\psi^{(i)}(r)\right) T^{i+j}
$$

So by comparing the coefficients of $T^{i+j}$ one sees that condition (iii) is fulfilled if and only if $\tilde{\psi} \circ\left(a . \mathrm{d}_{\hat{\Omega}}\right)=(a . \psi[[T]]) \circ \tilde{\psi}$ holds for arbitrary $a \in K \backslash\{0\}$ (e.g. $a=1$, i. e. condition (ii)). Moreover, this is fulfilled if and only if for all $i, j \in \mathbb{N}$ we have $\psi^{(j)} \circ \psi^{(i)}=\left(\begin{array}{c}i+j \\ j\end{array}\right) \psi^{(i+j)}$, i. e. $\psi$ is iterative.

Furthermore, we get for all $a, b \in K$ :

$$
((a . \psi)(b . \psi))^{(k)}=\sum_{i+j=k}(a . \psi)^{(i)} \circ(b . \psi)^{(j)}=\sum_{i+j=k} a^{i} b^{j} \psi^{(i)} \circ \psi^{(j)},
$$

since $b \in K$, and

$$
((a+b) \cdot \psi)^{(k)}=(a+b)^{k} \psi^{(k)}=\sum_{i+j=k} a^{i} b^{j}\left(\begin{array}{c}
i+j \\
i
\end{array}\right) \psi^{(i+j)} .
$$

So if $\psi$ is iterative, condition (iv) is fulfilled. If $\# K=\infty$, we obtain from condition (iv) that $\psi$ is iterative by comparing the coefficients of $a^{i}$.

If $\# K<\infty$, the following example shows that condition (iv) doesn't imply the others.

Example 5.5. Condition (iv) is in fact weaker if $K$ is finite. If for example $K=\mathbb{F}_{q}$ and $R=\mathbb{F}_{q}[t]$, then $\psi \in \operatorname{HD}_{K}(R)$ defined by $\psi(t)=t+1 \cdot T^{2 q-1}$ is not iterative, since

$$
(2 q-1) \psi^{(2 q-1)}(t)=2 q-1 \neq 0=\psi^{(2 q-2)}\left(\psi^{(1)}(t)\right) .
$$


On the other hand, for all $a \in \mathbb{F}_{q}$ we have $a^{2 q-1}=a$, and so

$$
\begin{aligned}
((a . \psi)(b . \psi))^{(k)}(t) & =\sum_{i+j=k} a^{i} b^{j} \psi^{(i)}\left(\psi^{(j)}(t)\right) \\
& =a^{k} \psi^{(k)}(t)+a^{k-2 q+1} b^{2 q-1} \psi^{(k-2 q+1)}(1) \\
& =\left\{\begin{array}{cc}
t & k=0 \\
a^{2 q-1}+b^{2 q-1}=(a+b)^{2 q-1} & k=2 q-1 \\
0 & \text { otherwise }
\end{array}\right\} \\
& =((a+b) \cdot \psi)^{(k)}(t)
\end{aligned}
$$

for all $a, b \in K=\mathbb{F}_{q}$.

Remark 5.6. Condition (iv) is very useful for calculations - even if $K$ is finite. If one has to show that some higher derivation $\psi \in \operatorname{HD}_{K}(R)$ is iterative, one can often use the following trick:

Let $\tilde{R}:=K^{\mathrm{sep}} \otimes_{K^{\mathrm{sep}} \cap R} R$ be the maximal separable extension of $R$ by constants. Then by Proposition 3.7 the higher derivation $\psi$ uniquely extends to a higher derivation $\psi_{e} \in \operatorname{HD}_{K}(\tilde{R})=\operatorname{HD}_{K^{\text {sep }}}(\tilde{R})$. Since $\# K^{\text {sep }}=\infty$, we can use condition (iv) to show that $\psi_{e}$ is iterative and therefore $\psi$ is iterative.

Whenever it will be shown that for all $a, b \in K^{\mathrm{sep}},(a . \psi)(b . \psi)=(a+b) \cdot \psi$, this trick will be used, although we won't mention it explicitly.

Lemma 5.7. Let $\phi \in \operatorname{ID}_{K}(R)$ be an iterative derivation and $\Psi \in \operatorname{HD}(M, \phi)$ be $a \phi$-derivation. Then $\Psi$ is iterative if and only if for all $a, b \in K^{\mathrm{sep}}$ the identity $(a . \Psi)(b . \Psi)=(a+b) . \Psi$ holds.

Proof. Analogous to the proof of Lemma 5.4

The next lemma states some structural properties of $\operatorname{ID}_{K}(R)$.

Lemma 5.8. (1) If two iterative derivations $\phi_{1}, \phi_{2} \in \operatorname{ID}_{K}(R)$ commute, i.e. they satisfy $\phi_{1}^{(i)} \circ \phi_{2}^{(j)}=\phi_{2}^{(j)} \circ \phi_{1}^{(i)}$ for all $i, j \in \mathbb{N}$, then $\phi_{1} \phi_{2}$ is again an iterative derivation.

(2) $\operatorname{ID}_{K}(R)$ is stable under the action of $K$.

(3) If $\tilde{R} \supset R$ is a ring extension such that every higher derivation on $R$ uniquely extends to a higher derivation on $\tilde{R}$ (e.g. if $\tilde{R}$ is formally étale over $R$ ), then the extension $\phi_{e} \in \operatorname{HD}_{K}(\tilde{R})$ of an iterative derivation $\phi \in \operatorname{ID}_{K}(R)$ is again iterative.

Proof. A short calculation shows that all occurring higher derivations satisfy condition (iv) of Lemma 5.4 and hence are iterative.

We have already seen that $\mathrm{d}_{\hat{\Omega}}$ satisfies the condition $\mathrm{d}_{\hat{\Omega}}^{(i)} \circ \mathrm{d}_{\hat{\Omega}}^{(j)}=\left(\begin{array}{c}i+j \\ i\end{array}\right) \mathrm{d}_{\hat{\Omega}}^{(i+j)}$ and that for iterative derivations $\phi \in \operatorname{ID}_{K}(R)$ we have the "same" condition $\phi^{(i)} \circ \phi^{(j)}=$ $\left(\begin{array}{c}i+j \\ i\end{array}\right) \phi^{(i+j)}$. This motivates the following definition of an iterative connection.

Definition 5.9. A higher connection $\nabla$ on $M$ is called an iterative connection if the identity

$$
\hat{\Omega}^{(i)} \circ{ }_{\hat{\Omega}} \nabla^{(j)}=\left(\begin{array}{c}
i+j \\
i
\end{array}\right) \hat{\Omega} \nabla^{(i+j)}
$$

holds for all $i, j \in \mathbb{N}$. (As defined in Section 2 for the general case, ${ }_{\Omega} \nabla^{(i)}$ denotes the part of ${ }_{\hat{\Omega}} \nabla$ that "increases degrees by $i$ ".) 
An iterative connection $\nabla$ on $M$ is called an integrable iterative connection if for all higher derivations $\psi_{1}, \psi_{2} \in \operatorname{HD}_{K}(R)$ we have $\nabla_{\psi_{1} \psi_{2}}=\nabla_{\psi_{1}} \nabla_{\psi_{2}}$.

The notion of an integrable iterative connection is motivated by the correspondence to the integrable (ordinary) connections in characteristic zero (cf. Section 7).

Theorem 5.10. Let $\nabla$ be a higher connection on $M$. Then:

(1) $\nabla$ is iterative if and only if for all $a, b \in K^{\mathrm{sep}}, a_{\hat{\Omega}_{\hat{\Omega}}} \nabla \circ b_{\hat{\Omega}_{\hat{\Omega}}} \nabla=(a+b) \cdot{ }_{\hat{\Omega}} \nabla$, and if and only if for all $a, b \in K^{\mathrm{sep}}, a_{\hat{\Omega}} \nabla \circ b . \nabla=(a+b) . \nabla$.

(2) If $\nabla$ is iterative, then for all iterative derivations $\phi \in \operatorname{ID}_{K}(R)$ the $\phi$ derivation $\nabla_{\phi}$ is again iterative. If $R$ has enough iterative derivations, i.e. if for every nonzero $\omega \in \hat{\Omega}_{R / K}$ there exists an iterative derivation $\phi \in \operatorname{ID}_{K}(R)$ such that $\tilde{\phi}(\omega) \neq 0$, then the converse is also true (where $\tilde{\phi}: \hat{\Omega}_{R / K} \rightarrow R[[T]]$ is the unique homomorphism of cgas satisfying $\phi=\tilde{\phi} \circ \mathrm{d}$; cf. Theorem 3.10).

Proof. The first statement is seen by a calculation similar to the one in Lemma 5.4. For proving the second part, let $\phi \in \operatorname{ID}_{K}(R)$ and consider the following diagram:

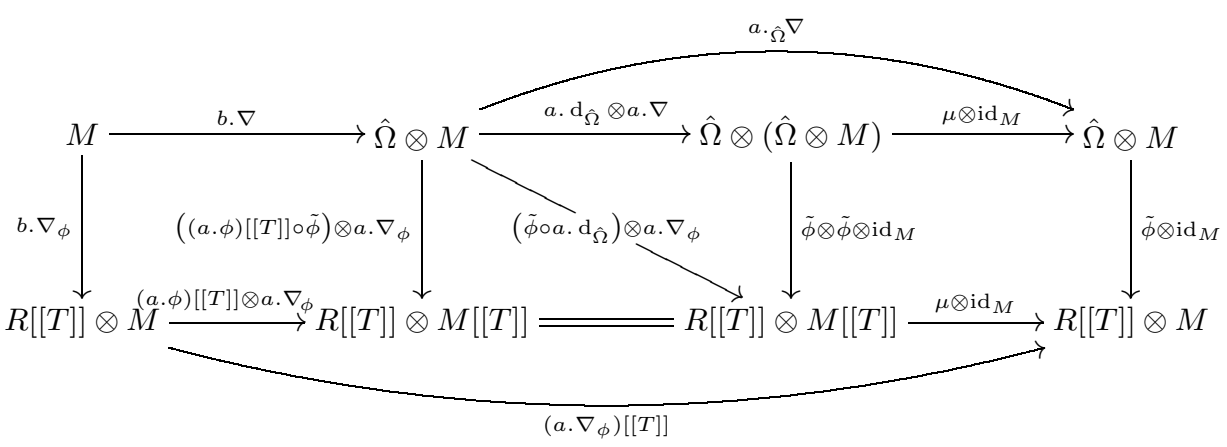

where the tensor product $R[[T]] \otimes M[[T]]$ is taken over a. $\phi(R)$, and $\hat{\Omega} \otimes(\hat{\Omega} \otimes M)$ means $\hat{\Omega} \otimes_{a . \mathrm{d}(R)}\left(\hat{\Omega} \otimes_{R} M\right)$. The other tensor products are taken over $R$.

The square on the left commutes, since

$$
b . \nabla_{\phi}=b .\left(\left(\tilde{\phi} \otimes \operatorname{id}_{M}\right) \circ \nabla\right)=\left(\tilde{\phi} \otimes \operatorname{id}_{M}\right) \circ(b . \nabla) .
$$

The lower triangle commutes by Lemma 5.4, since $\phi$ is iterative. The upper triangle commutes, since $a . \nabla_{\phi}=\left(\tilde{\phi} \otimes \operatorname{id}_{M}\right) \circ(a . \nabla)$, and the square on the right commutes, since $\tilde{\phi}$ is a homomorphism of algebras. Furthermore, the top of the diagram commutes by definition of $a_{\cdot \hat{\Omega}} \nabla$ and the bottom commutes, since $a \cdot \nabla_{\phi}$ is a $(a . \phi)$ derivation.

So the whole diagram commutes, and we obtain

$$
\left(\tilde{\phi} \otimes \operatorname{id}_{M}\right) \circ\left(a \cdot \hat{\Omega}_{\hat{\Omega}} \nabla\right) \circ(b . \nabla)=\left(a . \nabla_{\phi}\right)[[T]] \circ\left(b . \nabla_{\phi}\right)=\left(a . \nabla_{\phi}\right)\left(b . \nabla_{\phi}\right)
$$

for all iterative derivations $\phi \in \operatorname{ID}_{K}(R)$.

If $\nabla$ is iterative, we get

$$
(a+b) \cdot \nabla_{\phi}=\left(\tilde{\phi} \otimes \operatorname{id}_{M}\right) \circ(a+b) \cdot \nabla=\left(\tilde{\phi} \otimes \operatorname{id}_{M}\right) \circ\left(a \cdot \hat{\Omega}_{\Omega} \nabla\right) \circ(b \cdot \nabla)=\left(a \cdot \nabla_{\phi}\right)\left(b \cdot \nabla_{\phi}\right)
$$

by the first part of this theorem, and so by Lemma $5.7 \nabla_{\phi}$ is iterative. 
In turn, from the commuting diagram we see that if $\nabla_{\phi}$ is iterative for an iterative derivation $\phi \in \operatorname{ID}_{K}(R)$, we get

$$
\left(\tilde{\phi} \otimes \operatorname{id}_{M}\right) \circ(a \cdot \hat{\Omega} \nabla) \circ(b . \nabla)=\left(\tilde{\phi} \otimes \operatorname{id}_{M}\right) \circ(a+b) . \nabla
$$

for this $\phi$. So if $R$ has enough iterative derivations and $\nabla_{\phi}$ is iterative for all $\phi \in \operatorname{ID}_{K}(R)$, we obtain $\left(a_{\cdot} \hat{\Omega}\right) \circ(b . \nabla)=(a+b) . \nabla$, i. e. $\nabla$ is iterative.

\section{The Tannakian Category of modules With iterative CONnection}

In this section we show - assuming a slight restriction on the ring $R$ - that the finitely generated projective modules (i.e. locally free of finite rank) with higher connection form an abelian category and that the modules with iterative, resp. integrable iterative, connection form full subcategories. Furthermore, all of these categories form tensor categories over $K$ (in the sense of [Del90]); in fact they even form Tannakian categories.

Notation. From now on let $R$ be a regular ring over $K$ which is the localisation of a finitely generated $K$-algebra, such that $K$ is algebraically closed in $R$. Also keep in mind that we assumed $R$ to be an integral domain and $K$ to be a perfect field.

Furthermore, in the following a pair $(M, \nabla)$ always denotes a finitely generated $R$-module $M$ together with a higher connection $\nabla: M \rightarrow \hat{\Omega} \otimes_{R} M$, even if "finitely generated" is not mentioned. We recall the fact given in Corollary 4.5 , namely that such a module is always projective.

Definition 6.1. Let $\left(M_{1}, \nabla_{1}\right)$ and $\left(M_{2}, \nabla_{2}\right)$ be $R$-modules with higher connection. Then we call $f \in \operatorname{Hom}_{R}\left(M_{1}, M_{2}\right)$ a morphism of modules with higher connection, or a morphism for short, if the diagram

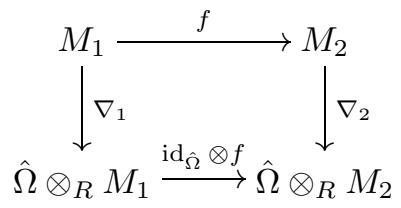

commutes. The set of all morphisms $f \in \operatorname{Hom}_{R}\left(M_{1}, M_{2}\right)$ will be denoted by $\operatorname{Mor}\left(\left(M_{1}, \nabla_{1}\right),\left(M_{2}, \nabla_{2}\right)\right)$. If the higher connections are clear from the context, we will sometimes omit them.

Remark 6.2. It is clear that the set of modules with higher connection, together with the sets of morphisms defined above, forms a category. This category will be denoted by $\operatorname{HCon}(R / K)$. Furthermore, the full subcategories of modules with iterative connection, resp. integrable iterative connection, will be denoted by $\operatorname{ICon}(R / K)$, resp. ICon $_{\mathbf{i n t}}(R / K)$, and by definition we have a chain of inclusions

$$
\operatorname{HCon}(R / K) \supset \operatorname{ICOn}(R / K) \supset \operatorname{ICon}_{\text {int }}(R / K) \text {. }
$$

As the objects of $\mathbf{H C o n}(R / K)$ are modules with an extra structure and the morphisms are special homomorphisms, we have a forgetful functor $\boldsymbol{\omega}: \operatorname{HCon}(R / K) \rightarrow$ $\operatorname{Mod}(R)$, which is faithful.

Definition 6.3. Let $\left(M_{1}, \nabla_{1}\right)$ and $\left(M_{2}, \nabla_{2}\right)$ be $R$-modules with higher connection. Then we define a higher connection $\nabla_{\oplus}$ on $\left(M_{1} \oplus M_{2}\right)$ by

$$
\nabla_{\oplus}: M_{1} \oplus M_{2} \stackrel{\nabla_{1} \oplus \nabla_{2}}{\longrightarrow} \hat{\Omega} \otimes M_{1} \oplus \hat{\Omega} \otimes M_{2} \cong \hat{\Omega} \otimes\left(M_{1} \oplus M_{2}\right)
$$


and a higher connection $\nabla_{\otimes}$ on $M_{1} \otimes_{R} M_{2}$ by

$$
\begin{aligned}
\nabla_{\otimes}: M_{1} \otimes_{R} M_{2} \stackrel{\nabla_{1} \otimes \nabla_{2}}{\longrightarrow}\left(\hat{\Omega} \otimes_{R} M_{1}\right) \otimes_{d(R)}\left(\hat{\Omega} \otimes_{R} M_{2}\right) \\
\stackrel{\cong}{\longrightarrow}\left(\hat{\Omega} \otimes_{d(R)} \hat{\Omega}\right) \otimes_{R}\left(M_{1} \otimes_{R} M_{2}\right) \stackrel{\mu \otimes \mathrm{id}}{\longrightarrow} \hat{\Omega} \otimes_{R}\left(M_{1} \otimes_{R} M_{2}\right) .
\end{aligned}
$$

Furthermore, we define a higher connection $\nabla_{H}$ on $\operatorname{Hom}_{R}\left(M_{1}, M_{2}\right)$ by the following:

For $f \in \operatorname{Hom}_{R}\left(M_{1}, M_{2}\right)$ the composition

$$
1 \otimes M_{1} \hookrightarrow \hat{\Omega} \otimes_{R} M_{1} \stackrel{\left({ }_{\hat{\Omega}} \nabla_{1}\right)^{-1}}{\longrightarrow} \hat{\Omega} \otimes_{R} M_{1} \stackrel{\mathrm{id}_{\hat{\Omega}} \otimes f}{\longrightarrow} \hat{\Omega} \otimes_{R} M_{2} \stackrel{\hat{\Omega} \nabla_{2}}{\longrightarrow} \hat{\Omega} \otimes_{R} M_{2}
$$

is an element of $\operatorname{Hom}_{R}\left(M_{1}, \hat{\Omega} \otimes_{R} M_{2}\right)$, and we have a natural isomorphism

$$
\operatorname{Hom}_{R}\left(M_{1}, \hat{\Omega} \otimes_{R} M_{2}\right) \cong \hat{\Omega} \otimes_{R} \operatorname{Hom}_{R}\left(M_{1}, M_{2}\right) .
$$

In this sense we define

$$
\nabla_{H}(f):=\left.{ }_{\hat{\Omega}} \nabla_{2} \circ\left(\operatorname{id}_{\hat{\Omega}} \otimes f\right) \circ\left({ }_{\hat{\Omega}} \nabla_{1}\right)^{-1}\right|_{1 \otimes M} .
$$

Remark 6.4. (i) If $\nabla_{1}$ is an iterative connection, the definition of $\nabla_{H}$ coincides with the definition given in Rös07], because then we have $\left.\left({ }_{\Omega} \nabla_{1}\right)^{-1}\right|_{1 \otimes M_{1}}=$ $-\left.{ }_{\Omega} \nabla_{1}\right|_{1 \otimes M_{1}}=-\nabla_{1}$.

(ii) As the referee pointed out, in the definition of the tensor product it might be possible to replace the twisting isomorphism $\left(\hat{\Omega} \otimes_{R} M_{1}\right) \otimes_{d(R)}\left(\hat{\Omega} \otimes_{R} M_{2}\right) \cong$ $\left(\hat{\Omega} \otimes_{d(R)} \hat{\Omega}\right) \otimes_{R}\left(M_{1} \otimes_{R} M_{2}\right)$ by a more general isomorphism in order to obtain a noncommutative theory. This might lead to an iterative variant of Y. André's framework for the theory of differential and difference equations (cf. And01]) and also to a more abstract framework for the iterative $q$ difference theory of C. Hardouin (cf. Hard08]).

Theorem 6.5. The category $\operatorname{HCon}(R / K)$ is an abelian category, and the categories $\mathbf{I C o n}(R / K)$ and $\mathbf{I C o n}_{\mathbf{i n t}}(R / K)$ are abelian subcategories.

Proof. For all $\left(M_{1}, \nabla_{1}\right),\left(M_{2}, \nabla_{2}\right) \in \mathbf{H C o n}(R / K)$ the set of morphisms $\operatorname{Mor}\left(M_{1}, M_{2}\right)$ is a subgroup of $\operatorname{Hom}_{R}\left(M_{1}, M_{2}\right)$, and so it is an abelian $\operatorname{group}$. Since $\operatorname{Mod}(R)$ is an abelian category, it is sufficient to show that kernels, direct sums and so on in the category $\operatorname{Mod}(R)$ can be equipped with a higher connection, resp. (integrable) iterative connection, and that all necessary homomorphisms (like the inclusion map of the kernel into the module) are morphisms.

The only point at which one has to be careful is the kernel: For a morphism $f \in \operatorname{Mor}\left(M_{1}, M_{2}\right)$, the higher connection $\nabla_{1}$ induces a map $\left.\nabla_{1}\right|_{\operatorname{Ker}(f)}: \operatorname{Ker}(f) \rightarrow$ $\operatorname{Ker}\left(\operatorname{id}_{\hat{\Omega}} \otimes f\right)$, and one has to verify that $\operatorname{Ker}\left(\operatorname{id}_{\hat{\Omega}} \otimes f\right)=\hat{\Omega} \otimes \operatorname{Ker}(f)$. But this follows from the fact that $\hat{\Omega}_{k}$ is a projective $R$-module for all $k \in \mathbb{N}$, and hence $\hat{\Omega} \otimes-$ is an exact functor. (See Rös07], Ch. 4.1, for details.)

Now we will show that these categories are tensor categories over $K$ (we will use the definition of a tensor category over $K$ given in [Del90]). By the previous theorem, they are all abelian, and by Corollary 4.5, all modules that arise are projective and the category $\operatorname{Proj-\operatorname {Mod}}(R)$ of finitely generated projective $R$-modules is known to satisfy all properties of a tensor category apart from being an abelian category. 
So we define

- the tensor product of $\left(M_{1}, \nabla_{1}\right)$ and $\left(M_{2}, \nabla_{2}\right)$ by

$$
\left(M_{1}, \nabla_{1}\right) \otimes\left(M_{2}, \nabla_{2}\right):=\left(M_{1} \otimes_{R} M_{2}, \nabla_{\otimes}\right)
$$

(this tensor product is obviously associative and commutative),

- the unital object $\mathbf{1}:=\left(R, \mathrm{~d}_{R}\right) \quad\left(R \otimes_{R} M \rightarrow M, r \otimes m \mapsto r m\right.$ is easily seen to be a morphism for all $(M, \nabla) \in \mathbf{H C o n}(R / K))$,

- the dual object to $(M, \nabla)$ by

$$
(M, \nabla)^{*}:=\left(M^{*}, \nabla^{*}\right)
$$

where $\nabla^{*}(f):=\mathrm{d}_{\hat{\Omega}} \circ\left(\operatorname{id}_{\hat{\Omega}} \otimes f\right) \circ\left(\hat{\Omega}^{-1} \nabla_{1 \otimes M} \in \operatorname{Hom}_{R}(M, \hat{\Omega})\right.$ for $f \in M^{*}=$ $\operatorname{Hom}_{R}(M, R)$

(here we used the fact that $\hat{\Omega} \otimes_{R} R \cong \hat{\Omega}$ and $\operatorname{Hom}_{R}(M, \hat{\Omega}) \cong \operatorname{Hom}_{R}(M, R) \otimes$ $\hat{\Omega}$. Cf. the definition of $\nabla_{H}$ in Definition 6.31),

- the internal hom object of $\left(M_{1}, \nabla_{1}\right)$ and $\left(M_{2}, \nabla_{2}\right)$ by

$$
\underline{\operatorname{Hom}}\left(\left(M_{1}, \nabla_{1}\right),\left(M_{2}, \nabla_{2}\right)\right):=\left(\operatorname{Hom}_{R}\left(M_{1}, M_{2}\right), \nabla_{H}\right) .
$$

Furthermore, we recognize that every endomorphism in $\operatorname{End}(\mathbf{1})$ is given by the image of $1 \in R$, which has to be constant. Since all constants are algebraic over $K$ and $K$ is algebraically closed in $R, \operatorname{End}(\mathbf{1})$ is isomorphic to $K$.

Lemma 6.6. For all $\left(M_{1}, \nabla_{1}\right),\left(M_{2}, \nabla_{2}\right) \in \mathbf{H C o n}(R / K)$ the isomorphism of $R$ modules,

$$
\iota_{M_{1}, M_{2}}: M_{1}^{*} \otimes_{R} M_{2} \rightarrow \operatorname{Hom}_{R}\left(M_{1}, M_{2}\right), \quad f \otimes m \mapsto\{v \mapsto f(v) \cdot m\},
$$

is a morphism (and therefore an isomorphism) in $\mathbf{H C o n}(R / K)$.

Proof. For all $f \otimes m \in M_{1}^{*} \otimes_{R} M_{2}$ and for all $v \in M_{1}$, we have

$$
\begin{aligned}
\nabla_{H}\left(\iota_{M_{1}, M_{2}}(f \otimes m)\right)(v) \\
=\left({ }_{\hat{\Omega}} \nabla_{2} \circ\left(\operatorname{id}_{\hat{\Omega}} \otimes \iota_{M_{1}, M_{2}}(f \otimes m)\right) \circ\left({ }_{\hat{\Omega}} \nabla_{1}^{-1}\right)\right)(1 \otimes v) \\
={ }_{{ }_{\Omega}} \nabla_{2}\left(\left(\operatorname{id}_{\hat{\Omega}} \otimes f\right)\left({ }_{\hat{\Omega}_{1}} \nabla_{1}^{-1}(1 \otimes v)\right) \cdot(1 \otimes m)\right) \\
=\mathrm{d}_{\hat{\Omega}}\left(\left(\operatorname{id}_{\hat{\Omega}} \otimes f\right)\left({ }_{\hat{\Omega}} \nabla_{1}^{-1}(1 \otimes v)\right)\right) \cdot \nabla_{2}(m) \\
=\left(\operatorname{id}_{\hat{\Omega}} \otimes \iota_{M_{1}, M_{2}}\right)\left(\left(\mathrm{d}_{\hat{\Omega}} \circ\left(\operatorname{id}_{\hat{\Omega}} \otimes f\right) \circ\left(\left.{ }_{\hat{\Omega}} \nabla_{1}^{-1}\right|_{1 \otimes M}\right)\right) \otimes \nabla_{2}(m)\right)(v) \\
=\left(\operatorname{id}_{\hat{\Omega}} \otimes \iota_{M_{1}, M_{2}}\right)\left(\nabla_{\otimes}(f \otimes m)\right)(v) .
\end{aligned}
$$

So $\nabla_{H} \circ \iota_{M_{1}, M_{2}}=\left(\operatorname{id}_{\hat{\Omega}} \otimes \iota_{M_{1}, M_{2}}\right) \circ \nabla_{\otimes}$, i.e. $\iota_{M_{1}, M_{2}}$ is a morphism.

Lemma 6.7. Let $(M, \nabla) \in \operatorname{HCon}(R / K)$, and let $\varepsilon_{M}: M \otimes M^{*} \rightarrow R$ and $\delta_{M}:$ $R \rightarrow M^{*} \otimes M$ be the evaluation and coevaluation homomorphisms given in the definition of a tensor category, i. e., $\varepsilon_{M}(m \otimes f)=f(m)$ and $\delta_{M}(1)=\iota_{M, M}^{-1}\left(\operatorname{id}_{M}\right)$. Then $\varepsilon_{M}$ and $\delta_{M}$ are morphisms in $\operatorname{HCon}(R / K)$. 
Proof. For $m \otimes f \in M \otimes M^{*}$, we have

$$
\begin{aligned}
\left(\operatorname{id}_{\hat{\Omega}} \otimes\right. & \left.\varepsilon_{M}\right)\left(\nabla_{\otimes}(m \otimes f)\right) \\
& =\left(\operatorname{id}_{\hat{\Omega}} \otimes \varepsilon_{M}\right)\left((\mu \otimes \mathrm{id}) \circ\left(\nabla(m) \otimes\left(\left.\mathrm{d}_{\hat{\Omega}} \circ\left(\mathrm{id}_{\hat{\Omega}} \otimes f\right) \circ{ }_{\hat{\Omega}} \nabla^{-1}\right|_{1 \otimes M}\right)\right)\right. \\
& =(\mu \otimes \mathrm{id})\left(\left(\mathrm{id}_{\hat{\Omega}} \otimes\left(\left.\mathrm{d}_{\hat{\Omega}} \circ\left(\mathrm{id}_{\hat{\Omega}} \otimes f\right) \circ \hat{\Omega} \nabla^{-1}\right|_{1 \otimes M}\right)\right)(\nabla(m))\right) \\
& =\left((\mu \otimes \mathrm{id}) \circ\left(\mathrm{d}_{\hat{\Omega}} \otimes \mathrm{d}_{\hat{\Omega}}\right) \circ\left(\mathrm{id}_{\hat{\Omega} \otimes \hat{\Omega}} \otimes f\right) \circ\left(\left.\mathrm{d}_{\hat{\Omega}}^{-1} \otimes_{\hat{\Omega}} \nabla^{-1}\right|_{1 \otimes M}\right) \circ \nabla\right)(m) \\
& =\left(\mathrm{d}_{\hat{\Omega}} \circ\left(\mathrm{id}_{\hat{\Omega}} \otimes f\right) \circ\left(\hat{\Omega}^{-1}\right) \circ \nabla\right)(m) \\
& =\left(\mathrm{d}_{\hat{\Omega}} \circ\left(\mathrm{id}_{\hat{\Omega}} \otimes f\right)\right)(1 \otimes m) \\
& =\mathrm{d}_{R}(f(m))=\mathrm{d}_{R}\left(\varepsilon_{M}(m \otimes f)\right) .
\end{aligned}
$$

So $\varepsilon_{M}$ is a morphism.

Since $\iota_{M, M}$ is an isomorphism in $\operatorname{HCon}(R / K), \delta_{M}$ is a morphism if and only if $\iota_{M, M} \circ \delta_{M}$ is a morphism. Now

$$
\begin{gathered}
\nabla_{H}\left(\left(\iota_{M, M} \circ \delta_{M}\right)(1)\right)=\nabla_{H}\left(\operatorname{id}_{M}\right)=\left.{ }_{\hat{\Omega}} \nabla \circ\left(\operatorname{id}_{\hat{\Omega}} \otimes \operatorname{id}_{M}\right) \circ{ }_{\hat{\Omega}} \nabla^{-1}\right|_{1 \otimes M} \\
=\left.{ }_{\hat{\Omega}} \nabla \circ{ }_{\hat{\Omega}} \nabla^{-1}\right|_{1 \otimes M}=1 \otimes \operatorname{id}_{M}, \\
\left(\operatorname{id}_{\hat{\Omega}} \otimes\left(\iota_{M, M} \circ \delta_{M}\right)\right)\left(\mathrm{d}_{R}(1)\right)=\left(\operatorname{id}_{\hat{\Omega}} \otimes\left(\iota_{M, M} \circ \delta_{M}\right)\right)(1 \otimes 1)=1 \otimes \operatorname{id}_{M},
\end{gathered}
$$

so $\delta_{M}$ is a morphism.

Theorem 6.8. $\operatorname{HCon}(R / K), \operatorname{ICon}(R / K)$ and $\mathbf{I C o n}_{\text {int }}(R / K)$ are tensor categories over $K$.

Proof. Since we have already shown that these categories are abelian, that $\operatorname{HCon}(R / K)$ is equipped with an associative and commutative tensor product, and that $\varepsilon_{M}$ and $\delta_{M}$ are morphisms, we already know that $\operatorname{HCon}(R / K)$ is a tensor category. Hence, it only remains to show that the two subcategories are closed under tensor products and duals.

It is checked immediately that for $\left(M_{1}, \nabla_{1}\right),\left(M_{2}, \nabla_{2}\right) \in \mathbf{I C o n}(R / K)$ the higher connection $\nabla_{\otimes}$ on $M_{1} \otimes M_{2}$ satisfies $\left(a \cdot \hat{\Omega}_{\otimes}\right) \circ\left(b . \nabla_{\otimes}\right)=(a+b) . \nabla_{\otimes}$ for all $a, b \in K^{\mathrm{sep}}$. One also easily checks that the higher connection $\nabla_{1}^{*}$ on $M_{1}^{*}$ satisfies $\left(a_{._{\hat{\Omega}}} \nabla_{1}^{*}\right) \circ$ $\left(b . \nabla_{1}^{*}\right)=(a+b) \cdot \hat{\Omega}_{1}^{*}$ for all $a, b \in K^{\text {sep }}$, if $\nabla_{1}$ is iterative. Hence $\operatorname{ICon}(R / K)$ is a tensor category.

Assuming that $\left(M_{1}, \nabla_{1}\right),\left(M_{2}, \nabla_{2}\right) \in \mathbf{I C o n}_{\text {int }}(R / K)$, the integrability conditions for $\nabla_{\otimes}$ and $\nabla_{1}^{*}$ are obtained by a short calculation using the following lemma.

Lemma 6.9. Let $\left(M_{1}, \nabla_{1}\right),\left(M_{2}, \nabla_{2}\right) \in \mathbf{H C o n}(R / K)$ and let $\psi \in \operatorname{HD}_{K}(R)$. Then we have:

(1) $\left(\nabla_{\otimes}\right)_{\psi}=(\mu \otimes \mathrm{id}) \circ\left(\left(\nabla_{1}\right)_{\psi} \otimes\left(\nabla_{2}\right)_{\psi}\right)$.

(2) For all $f \in \operatorname{Hom}_{R}\left(M_{1}, M_{2}\right)$,

$$
\left(\left(\nabla_{H}\right)_{\psi}\right)(f)=\left.\left(\nabla_{2}\right)_{\psi}[[T]] \circ\left(\operatorname{id}_{R[[T]]} \otimes f\right) \circ\left(\left(\nabla_{1}\right)_{\psi}[[T]]\right)^{-1}\right|_{1 \otimes M} .
$$

Proof. We have

$$
\begin{aligned}
\left(\nabla_{\otimes}\right)_{\psi} & =(\tilde{\psi} \otimes \mathrm{id}) \circ(\mu \otimes \mathrm{id}) \circ\left(\nabla_{1} \otimes \nabla_{2}\right) \\
& =(\mu \otimes \mathrm{id}) \circ\left(\left(\tilde{\psi} \otimes \mathrm{id}_{M_{1}}\right) \otimes\left(\tilde{\psi} \otimes \mathrm{id}_{M_{2}}\right)\right) \circ\left(\nabla_{1} \otimes \nabla_{2}\right) \\
& =(\mu \otimes \mathrm{id}) \circ\left(\left(\nabla_{1}\right)_{\psi} \otimes\left(\nabla_{2}\right)_{\psi}\right)
\end{aligned}
$$


which shows the first part. For the proof of the second part, consider the following diagram:

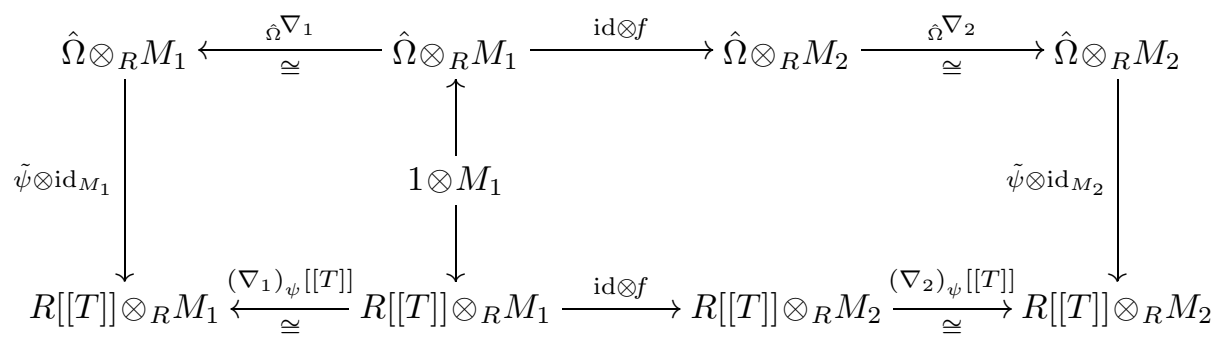

It is sufficient to show that both maps from the upper left corner of the diagram to the lower right corner are equal. Both parts of the diagram (starting at $1 \otimes M_{1}$ in the middle) commute by definition of $\left(\nabla_{1}\right)_{\psi}$, resp. $\left(\nabla_{2}\right)_{\psi}$. Furthermore, $\hat{\Omega} \otimes M_{1}$ is generated as an $\hat{\Omega}$-module by elements in $1 \otimes M_{1}$, and since ${ }_{\hat{\Omega}} \nabla_{1}$ is an isomorphism, $\hat{\Omega} \otimes M_{1}$ is also generated as an $\hat{\Omega}$-module by elements of the form ${ }_{\hat{\Omega}} \nabla_{1}(1 \otimes m)$ for $m \in M_{1}$. The equality of the maps then follows from the $\hat{\Omega}$-linearity of the upper row and the $R[[T]]$-linearity of the lower row.

Theorem 6.10. The categories $\operatorname{HCon}(R / K), \operatorname{ICon}(R / K)$ and $\operatorname{ICon}_{\text {int }}(R / K)$ are Tannakian categories with the forgetful functor $\boldsymbol{\omega}: \operatorname{HCon}(R / K) \rightarrow \operatorname{Mod}(R)$ (restricted to the respective category) as fibre functor. If, moreover, $R$ has a $K$ rational point, i.e., there exists a maximal ideal $\mathfrak{m} \unlhd R$ with $K \cong R / \mathfrak{m}$, then these categories are neutral Tannakian categories with fibre functor $\boldsymbol{\omega}_{K}: \operatorname{HCon}(R / K) \stackrel{\omega}{\longrightarrow}$ $\operatorname{Mod}(R) \stackrel{\otimes_{R} R / \mathfrak{m}}{\longrightarrow} \operatorname{Vect}(K)$.

Proof. By construction, the functor $\boldsymbol{\omega}$ is a fibre functor, and so the tensor categories $\operatorname{HCon}(R / K), \operatorname{ICon}(R / K)$ and $\mathbf{I C o n}_{\text {int }}(R / K)$ are Tannakian categories. If $R$ has a $K$-rational point, by Del90, 2.8, $\boldsymbol{\omega}_{K}$ is a fibre functor. This proves the second part.

By Tannakian duality, every neutral Tannakian category $\mathcal{T}$ over $K$ with fibre functor $\boldsymbol{\omega}_{K}$ is equivalent to the category of finite dimensional representations of a group scheme defined over $K$, called the Tannaka group scheme (or fundamental group scheme $\Pi\left(\mathcal{T}, \boldsymbol{\omega}_{K}\right)$ of $\mathcal{T}$. Furthermore, this group scheme is the projective limit of all quotients $\left.G_{V}:=\Pi(\langle\langle V\rangle\rangle),\left.\omega_{K}\right|_{\langle\langle V\rangle\rangle}\right)$, where $V$ ranges over all objects of $\mathcal{T}$ and $\langle\langle V\rangle\rangle$ denotes the smallest Tannakian subcategory of $\mathcal{T}$ that contains $V$.

Using the Picard-Vessiot theory in the second part of this paper, we obtain the following proposition:

Proposition 6.11. Let $R$ have a $K$-rational point and let $K$ be algebraically closed. Then the Tannaka group schemes $\Pi\left(\mathbf{I C o n}(R / K), \boldsymbol{\omega}_{K}\right)$ and $\Pi\left(\mathbf{I C o n}_{\mathbf{i n t}}(R / K), \boldsymbol{\omega}_{K}\right)$ are reduced group schemes.

Proof. If $\operatorname{char}(K)=0$, then by general theory all group schemes are reduced, and nothing has to be shown. So assume $\operatorname{char}(K)=p>0$.

Using the equivalence of categories given in Theorem 8.7 and the identification in Remark 8.3, we obtain from [San07, 2.3.1, that the Tannaka group scheme associated to $\mathbf{I} \operatorname{Con}_{\text {int }}(R / K)$ is reduced, even a perfect group scheme. (The reducedness also follows from the reducedness of $\Pi\left(\operatorname{ICon}(R / K), \boldsymbol{\omega}_{K}\right)$, since $\Pi\left(\mathbf{I C o n}_{\mathbf{i n t}}(R / K), \boldsymbol{\omega}_{K}\right)$ is a quotient.) 
Since $\Pi\left(\operatorname{ICon}(R / K), \boldsymbol{\omega}_{K}\right)$ is the projective limit of all $G_{(M, \nabla)}:=\Pi(\langle\langle(M, \nabla)\rangle\rangle$, $\left.\left.\boldsymbol{\omega}_{K}\right|_{\langle\langle(M, \nabla)\rangle\rangle}\right)$, it suffices to show that all $G_{(M, \nabla)}$ are reduced. Let $F$ be the quotient field of $R$ and $E / F$ be a PPV-extension for $F \otimes_{R} M$ as defined in Section 10, which exists since $K$ is algebraically closed (cf. Remark 10.6). Then $G_{(M, \nabla)}$ is isomorphic to the Galois group scheme $\operatorname{Gal}(E / F)$ (cf. Remark 10.13). By Proposition 8.1, we have $\operatorname{Ker}\left(\mathrm{d}_{F}^{(1)}\right)=F^{p}$, and hence by Corollary 11.7, $\operatorname{Gal}(E / F) \cong G_{(M, \nabla)}$ is reduced.

Remark 6.12. One might ask whether the inclusions in the chain of categories $\operatorname{HCon}(R / K) \supset \operatorname{ICon}(R / K) \supset \operatorname{ICon}_{\text {int }}(R / K)$ are strict.

Clearly, $\operatorname{HCon}(R / K) \neq \operatorname{ICon}(R / K)$, because if for example $M$ is a free $R$ module of dimension 1 with basis $b_{1} \in M$, every $\omega=\sum_{j=0}^{\infty} \omega_{j} \in \hat{\Omega}_{R / K}$ with $\omega_{0}=1$ defines a higher connection $\nabla: M \rightarrow \hat{\Omega}_{R / K} \otimes_{R} M, b_{1} \mapsto \omega \otimes b_{1}$, but in general this higher connection is not iterative, because if $\nabla$ is iterative, $\omega$ satisfies the condition

$$
0=\left(-{ }_{\hat{\Omega}} \nabla \circ \nabla\right)^{(2)}\left(b_{1}\right)=\left(2 \omega_{2}-\omega_{1}^{2}+\mathrm{d}_{\hat{\Omega}}^{(1)}\left(\omega_{1}\right)\right) \otimes b_{1} .
$$

(The only exception is the case when $R$ is algebraic over $K$, because in this case $\hat{\Omega}_{R / K}=R$ and hence all categories above are equivalent to $\operatorname{Mod}(R)$ ).

The inclusion $\operatorname{ICon}(R / K) \supset \mathbf{I C o n}_{\mathbf{i n t}}(R / K)$ is also strict in general, because in the next section we will see that in characteristic zero, the category $\operatorname{ICon}(R / K)$ is equivalent to the category of modules with (ordinary) connection over $R$ and ICon $_{\text {int }}(R / K)$ is equivalent to the category of modules with integrable connection over $R$, and it is known that those two categories are different if for example $R=$ $K\left(t_{1}, t_{2}\right)$. However, it is also known that every (ordinary) connection is integrable if $\operatorname{char}(K)=0$ and $R$ is an algebraic function field in one variable over $K$. In Section 8, we will see that also $\mathbf{I} \operatorname{Con}(R / K)=\mathbf{I C o n}_{\text {int }}(R / K)$ if $R$ is an algebraic function field in one variable over $K$ and $\operatorname{char}(K)=p$.

\section{Characteristic zero}

For $\operatorname{char}(K)=0$, in general one gets the usual constructions of derivations, differentials and connections by restricting to the terms of degree 1 . On the other hand these constructions can be uniquely extended to iterative derivations and iterative connections. Moreover, integrable connections, i.e. connections which preserve commutators of derivations, correspond to integrable iterative connections. This will be proven in this section.

So throughout this section, $K$ is a field of characteristic zero and $R$ is an integral domain which is a regular ring and the localisation of a finitely generated $K$-algebra. Furthermore, we assume that $R$ has a maximal ideal $\mathfrak{m} \unlhd R$, such that $R / \mathfrak{m}$ is a finite extension of $K . M$ denotes a finitely generated $R$-module.

\section{Proposition 7.1.}

(i) The map

$$
\operatorname{Der}(R / K) \longrightarrow \operatorname{ID}_{K}(R), \partial \mapsto \phi_{\partial},
$$

given by

$$
\phi_{\partial}(r):=\sum_{n=0}^{\infty} \frac{1}{n !} \partial^{n}(r) T^{n}
$$

for all $r \in R$, is a bijection, and the inverse map is given by $\phi \mapsto \phi^{(1)}$. 
For a given derivation $\partial$ on $R$ and the corresponding iterative derivation $\phi_{\partial}$, the map I : $\operatorname{Der}_{R}(M) \rightarrow \operatorname{ID}\left(M, \phi_{\partial}\right), \partial_{M} \mapsto \Phi_{\partial_{M}}$, given by

$$
\Phi_{\partial_{M}}(m):=\sum_{n=0}^{\infty} \frac{1}{n !} \partial_{M}^{n}(m) T^{n}
$$

for all $m \in M$, is a bijection, and the inverse map is given by $\Phi \mapsto \Phi^{(1)}$.

(ii) The $R$-module $\left(\hat{\Omega}_{R / K}\right)_{1}$ is canonically isomorphic to the module of (ordinary) differentials $\Omega_{R / K}$, and $\mathrm{d}^{(1)}: R \rightarrow\left(\hat{\Omega}_{R / K}\right)_{1} \cong \Omega_{R / K}$ is the universal derivation.

(iii) For every iterative connection $\nabla$ on $M$, the map $\nabla^{(1)}: M \rightarrow\left(\hat{\Omega}_{R / K}\right)_{1} \otimes$ $M \cong \Omega_{R / K} \otimes M$ is a connection on $M$, and every connection $\nabla^{(1)}$ on $M$ uniquely extends to an iterative connection on $M$. Furthermore, $\nabla$ is an integrable iterative connection if and only if $\nabla^{(1)}$ is an integrable connection.

Proof. i) Let $\partial \in \operatorname{Der}(R / K)$ be a derivation. Then for all $i, j \in \mathbb{N}: \frac{1}{i !} \partial^{i} \circ \frac{1}{j !} \partial^{j}=$ $\left(\begin{array}{c}i+j \\ i\end{array}\right) \frac{1}{(i+j) !} \partial^{i+j}$. So $\phi_{\partial}$ is an iterative derivation. On the other hand, for every iterative derivation $\phi$, one obtains $\phi^{(k)}=\frac{1}{k !}\left(\phi^{(1)}\right)^{k}$ for all $k \in \mathbb{N}$ by applying the formula $\phi^{(i)}=\frac{1}{i} \phi^{(1)} \circ \phi^{(i-1)}$ inductively. Finally by Remark 3.2 for all $r, s \in R$ we have $\phi^{(1)}(r s)=r \phi^{(1)}(s)+\phi^{(1)}(r) s$, i.e. $\phi^{(1)} \in \operatorname{Der}(R / K)$.

The bijection I : $\operatorname{Der}_{R}(M) \rightarrow \operatorname{ID}\left(M, \phi_{\partial}\right)$ is shown analogously.

ii) The construction of $\left(\hat{\Omega}_{R / K}\right)_{1}$ given in the proof of Theorem 3.10 is the same as the usual construction of $\Omega_{R / K}$ (e.g. in [Hart77, Ch. II.8).

iii) The bijection of the iterative connections and the ordinary connections is shown analogous to the first part. So we only prove the equivalence of the integrability conditions. Let $\partial_{1}, \partial_{2} \in \operatorname{Der}(R / K)$ be derivations, let $\phi_{i}:=\phi_{\partial_{i}}(i=1,2)$ be the corresponding iterative derivations, and let $\nabla$ be an iterative connection on $M$. By an explicit calculation one gets

$$
\left(\phi_{1} \phi_{2} \phi_{1}^{-1} \phi_{2}^{-1}\right)^{(1)}=0 \quad \text { and } \quad\left(\phi_{1} \phi_{2} \phi_{1}^{-1} \phi_{2}^{-1}\right)^{(2)}=\partial_{1} \circ \partial_{2}-\partial_{2} \circ \partial_{1}=\left[\partial_{1}, \partial_{2}\right] \text {. }
$$

From this and the iterativity condition of $\nabla$, one computes that

$$
\nabla_{\phi_{1} \phi_{2} \phi_{1}^{-1} \phi_{2}^{-1}}^{(2)}=\left(\nabla^{(1)}\right)_{\left[\partial_{1}, \partial_{2}\right]} .
$$

(The last expression means that we apply the ordinary connection $\nabla^{(1)}$ to the derivation $\left[\partial_{1}, \partial_{2}\right]$.) On the other hand, by quite the same calculation as before one obtains

$$
\left(\nabla_{\phi_{1}} \nabla_{\phi_{2}} \nabla_{\phi_{1}}^{-1} \nabla_{\phi_{2}}^{-1}\right)^{(2)}=\nabla_{\phi_{1}}^{(1)} \circ \nabla_{\phi_{2}}^{(1)}-\nabla_{\phi_{2}}^{(1)} \circ \nabla_{\phi_{1}}^{(1)}=\left[\left(\nabla^{(1)}\right)_{\partial_{1}},\left(\nabla^{(1)}\right)_{\partial_{2}}\right] .
$$

So if $\nabla$ is an integrable iterative connection, then

$$
\nabla_{\phi_{1} \phi_{2} \phi_{1}^{-1} \phi_{2}^{-1}}^{(2)}=\left(\nabla_{\phi_{1}} \nabla_{\phi_{2}} \nabla_{\phi_{1}}^{-1} \nabla_{\phi_{2}}^{-1}\right)^{(2)}
$$

and hence $\nabla^{(1)}$ is an integrable connection.

For the converse, consider the complete local ring $\hat{R}_{\mathfrak{m}}$. We first note that for an arbitrary $R$-cga $B$, every higher derivation $\psi \in \operatorname{HD}_{K}(R, B)$ can be extended canonically to a higher derivation $\psi_{e} \in \operatorname{HD}_{K}\left(\hat{R}_{\mathfrak{m}}, \hat{R}_{\mathfrak{m}} \otimes B\right)$ in the following way: Every homogeneous component $\psi^{(k)}(k \in \mathbb{N})$ can be uniquely extended to the localisation $R_{\mathfrak{m}}$ (see Proposition 3.7). This extension is continuous with respect to 
the $\mathfrak{m}$-adic topology, since for all $i \in \mathbb{N}, \psi^{(k)}\left(\mathfrak{m}^{i}\right) \subseteq \mathfrak{m}^{i-k}\left(R_{\mathfrak{m}} \otimes B_{k}\right)$. So $\psi^{(k)}$ can be uniquely extended to a map $\psi_{e}^{(k)}: \hat{R}_{\mathfrak{m}} \rightarrow \hat{R}_{\mathfrak{m}} \otimes B_{k}$, which is continuous with respect to the $\mathfrak{m}$-adic topology. One easily verifies that indeed $\psi_{e}:=\sum_{k=0}^{\infty} \psi_{e}^{(k)}$ : $\hat{R}_{\mathfrak{m}} \rightarrow \hat{R}_{\mathfrak{m}} \otimes B$ is a higher derivation.

Since the extension is canonical, we obtain the identities $\left(\operatorname{id}_{\hat{R}_{\mathfrak{m}}} \otimes \widetilde{\psi}\right) \circ \mathrm{d}_{R, e}=\psi_{e}$ $\left(\mathrm{d}_{R, e}\right.$ denotes the extension of the universal derivation $\left.\mathrm{d}_{R}\right)$ and $\left(\psi_{1} \psi_{2}\right)_{e}=\psi_{1, e} \psi_{2, e}$ for all $\psi_{1}, \psi_{2} \in \operatorname{HD}_{K}(R)$.

Now let $\nabla$ be an iterative connection such that $\nabla^{(1)}$ is an integrable connection. By Kat70, Prop. 8.9, the $\hat{R}_{\mathfrak{m}}$-module $\hat{R}_{\mathfrak{m}} \otimes_{R} M$ is a trivial differential module, i.e., there is an $\hat{R}_{\mathfrak{m}}$-basis $\boldsymbol{b}=\left(b_{1}, \ldots, b_{n}\right)$ of $\hat{R}_{\mathfrak{m}} \otimes_{R} M$ such that $\nabla^{(1)}(\boldsymbol{b})=0$, where $\nabla^{(1)}$ is extended to $\hat{R}_{\mathfrak{m}} \otimes_{R} M$ in the same manner as the higher derivations. Since $\nabla$ is iterative, this implies $\nabla^{(k)}(\boldsymbol{b})=0$ for all $k>0$.

Hence for all $\psi_{1}, \psi_{2} \in \operatorname{HD}_{K}(R)$ and all vectors $\boldsymbol{y} \in \hat{R}_{\mathfrak{m}}^{n}$ s.t. $\sum y_{i} b_{i} \in M$, we have

$$
\begin{aligned}
\nabla_{\psi_{1} \psi_{2}}\left(\sum y_{i} b_{i}\right) & =\left(\widetilde{\psi_{1} \psi_{2}} \otimes \mathrm{id}\right)\left(\sum \mathrm{d}_{R, e}\left(y_{i}\right) \nabla\left(b_{i}\right)\right) \\
& =\left(\widetilde{\psi_{1} \psi_{2}} \otimes \mathrm{id}\right)\left(\sum \mathrm{d}_{R, e}\left(y_{i}\right) \cdot b_{i}\right)=\sum\left(\psi_{1} \psi_{2}\right)_{e}\left(y_{i}\right) \cdot b_{i}
\end{aligned}
$$

and

$$
\nabla_{\psi_{1}} \nabla_{\psi_{2}}\left(\sum y_{i} b_{i}\right)=\sum \psi_{1, e}[[T]]\left(\psi_{2, e}\left(y_{i}\right)\right) \cdot b_{i}=\sum\left(\psi_{1} \psi_{2}\right)_{e}\left(y_{i}\right) \cdot b_{i} .
$$

Hence $\nabla_{\psi_{1} \psi_{2}}=\nabla_{\psi_{1}} \nabla_{\psi_{2}}$, i.e., $\nabla$ is an integrable iterative connection.

As a consequence of the previous proposition, we obtain

Theorem 7.2. Under the assumptions given above, the category $\operatorname{ICon}(R / K)$ (resp. ICon $\left._{\text {int }}(R / K)\right)$ of finitely generated $R$-modules with iterative connection (resp. integrable iterative connection) and the category of finitely generated $R$-modules with connection (resp. integrable connection) are equivalent.

We end this section with a comparison of integrable iterative connections and stratifications (cf. BO78, Def. 2.10): From the previous theorem and the fact that for a smooth ring in characteristic zero a stratification is equivalent to an integrable connection (cf. BO78, Thm 2.15 for a sketch of the proof), we deduce the following corollary. In the next section, we will see that the corollary also holds if the characteristic of $K$ is not zero (cf. Corollary 8.8).

Corollary 7.3. Let $R$ be smooth over $K$. Then the category $\operatorname{ICon}_{\mathbf{i n t}}(R / K)$ is equivalent to the category of stratified modules over $R$.

\section{Positive Characteristic}

In this section, we consider the case where $K$ has positive characteristic $p$. Contrary to characteristic zero, iterative derivations and iterative connections are no longer determined by the term of degree 1 . Moreover, not every derivation $\partial \in \operatorname{Der}(R / K)$ can be extended to an iterative derivation $\phi$ with $\phi^{(1)}=\partial$, because the conditions on an iterative derivation imply $\left(\phi^{(1)}\right)^{p}=p ! \cdot \phi^{(p)}=0$, i.e., at least $\partial$ has to be nilpotent.

But there are some other structural properties: The main result is that every module with integrable iterative connection gives rise to a projective system and vice versa, similar to the correspondence of projective systems and iterative differential modules over function fields given in Mat01, Ch. 2.2. In fact, when $R$ 
is an algebraic function field in one variable, the projective systems defined here are equal to those defined by Matzat and van der Put, which shows that in this case the categories $\mathbf{I C o n}(R / K), \mathbf{I C o n}_{\text {int }}(R / K), \operatorname{Proj}_{R}$ and $\mathbf{I D}_{R}$ are equivalent. $\left(\right.$ Here $\mathbf{P r o j}_{R}$ denotes the category of projective systems over $R$ and $\mathbf{I D}_{R}$ denotes the category of ID-modules as given in [Mat01.)

So in this section, let $K$ be a perfect field of characteristic $p>0$ and let $R$ be an integral domain which is a regular ring and the localisation of a finitely generated $K$-algebra. Furthermore let $t_{1}, \ldots, t_{m}$ denote a separable transcendence basis for $R$, i.e. $\operatorname{Quot}(R)$ is a separable algebraic extension of the rational function field $K\left(t_{1}, \ldots, t_{m}\right) \cdot 2$

$R$ has a natural sequence of $K$-subrings $\left(R_{l}\right)_{l \in \mathbb{N}}$ given by $R_{l}:=R^{p^{l}}$. The following proposition gives a characterisation of this sequence by the higher differential:

Proposition 8.1 (Frobenius compatibility). For all $l \in \mathbb{N}$,

$$
R_{l}=\bigcap_{0<j<p^{l}} \operatorname{Ker}\left(\mathrm{d}_{R}^{(j)}\right) .
$$

Proof. Since $\mathrm{d}_{R}$ is a homomorphism of algebras, $\mathrm{d}_{R}\left(R_{l}\right)=\mathrm{d}_{R}\left(R^{p^{l}}\right) \subset\left(\hat{\Omega}_{R / K}\right)^{p^{l}}$, and therefore $\mathrm{d}_{R}^{(j)}(r)=0\left(0<j<p^{l}\right)$ for all $r \in R_{l}$. The other inclusion is shown inductively: The case $l=0$ is trivial. Now let $r \in R$ satisfy $\mathrm{d}_{R}^{(j)}(r)=0$ for $0<j<p^{l}$. By induction hypothesis $r \in R_{l-1}$. So there exists $s \in R$ with $s^{p^{l-1}}=r$. If $s \notin R^{p}$, then $s$ is a separating element of $R$ and we can find separating variables $s=s_{1}, s_{2}, \ldots, s_{m}$ for $R$, i.e. $\operatorname{Quot}(R) / K\left(s_{1}, \ldots, s_{m}\right)$ is a finite separable extension. By applying Proposition 3.12 and Theorem $3.13(\mathrm{~b})$, we see that $\mathrm{d}_{R}^{(1)}(s) \neq 0$, and so

$$
0 \neq\left(\mathrm{d}_{R}^{(1)}(s)\right)^{p^{l-1}}=\mathrm{d}_{R}^{\left(p^{l-1}\right)}\left(s^{p^{l-1}}\right)=\mathrm{d}_{R}^{\left(p^{l-1}\right)}(r),
$$

which is a contradiction. So $s \in R^{p}$ and $r \in R_{l}$.

In the case of $R$ being an algebraic function field in one variable, it was shown by F. K. Schmidt (see [HS37, Thms. 10 and 15) that for an iterative derivation $\phi \in \operatorname{ID}_{K}(R)$ satisfying $\phi^{(1)} \neq 0$, we have $R^{p^{l}}=\bigcap_{0<j<p^{l}} \operatorname{Ker}\left(\phi^{(j)}\right)$. So in this case we obtain the same sequence of subfields when "only" looking at an iterative derivation instead of the universal derivation.

Definition 8.2. A Frobenius compatible projective system over $R$ (or an Fc-projective system over $R$ for short) is a sequence $\left(M_{l}, \varphi_{l}\right)_{l \in \mathbb{N}}$ with the following properties:

(1) For all $l \in \mathbb{N}, M_{l}$ is a finitely generated $R_{l}$-module.

(2) $\varphi_{l}: M_{l+1} \hookrightarrow M_{l}$ is a monomorphism of $R_{l+1}$-modules that extends to an isomorphism id $R_{l} \otimes \varphi_{l}: R_{l} \otimes_{R_{l+1}} M_{l+1} \rightarrow M_{l}$.

A morphism $\alpha:\left(M_{l}, \varphi_{l}\right) \rightarrow\left(M_{l}^{\prime}, \varphi_{l}^{\prime}\right)$ of Fc-projective systems over $R$ is a sequence $\alpha=\left(\alpha_{l}\right)_{l \in \mathbb{N}}$ of homomorphisms of modules $\alpha_{l}: M_{l} \rightarrow M_{l}^{\prime}$ satisfying $\varphi_{l}^{\prime} \circ \alpha_{l+1}=\alpha_{l} \circ \varphi_{l}$.

Remark 8.3. An Fc-projective system $\left(M_{l}, \varphi_{l}\right)_{l \in \mathbb{N}}$ over $R$ is nothing else than a flat bundle on $\operatorname{Spec}(R)$ (cf. Gie75], Def. 1.1), resp. an F-divided sheaf on $\operatorname{Spec}(R)$ (cf. San07, Def. 4), if one identifies $R_{l}=R^{p^{l}}$ with $R$ via the Frobenius homomorphism

${ }^{2}$ This includes the case $m=0$, although in this case everything becomes trivial. 
$\mathbf{F}_{l}: R \rightarrow R_{l}, x \mapsto x^{p^{l}}$. Then all $M_{l}$ can be regarded as $R$-modules and the maps $\operatorname{id}_{R_{l}} \otimes \varphi_{l}: R_{l} \otimes_{R_{l+1}} M_{l+1} \rightarrow M_{l}$ become $R$-linear isomorphisms $\sigma_{l}: \mathbf{F}_{1}^{*}\left(M_{l+1}\right) \rightarrow M_{l}$ from the Frobenius pullback of $M_{l+1}$ to $M_{l}$, i.e. $\left(M_{l}, \sigma_{l}\right)_{l \in \mathbb{N}}$ is a flat bundle on $\operatorname{Spec}(R)$.

Proposition 8.4. Every Fc-projective system $\left(M_{l}, \varphi_{l}\right)_{l \in \mathbb{N}}$ over $R$ defines an integrable iterative connection $\nabla$ on $M:=M_{0}$ satisfying

$$
\bigcap_{0<j<p^{l}} \operatorname{Ker}\left(\nabla^{(j)}\right)=\left(\varphi_{0} \circ \cdots \circ \varphi_{l-1}\right)\left(M_{l}\right) .
$$

For a morphism $\left(\alpha_{l}\right)_{l \in \mathbb{N}}:\left(M_{l}, \varphi_{l}\right) \rightarrow\left(M_{l}^{\prime}, \varphi_{l}^{\prime}\right)$ of Fc-projective systems over $R$, the homomorphism $\alpha_{0}: M=M_{0} \rightarrow M^{\prime}=M_{0}^{\prime}$ then is a morphism of modules with higher connection.

Proof. The proof is similar to the construction of a stratification related to a flat bundle in the proof of Gie75, Thm. 1.3.

In order to define $\nabla^{(k)}$, choose $l \in \mathbb{N}$ such that $p^{l}>k$ and let $\chi_{l}: R \otimes_{R_{l}} M_{l} \cong M$ be the composition of the isomorphisms $\operatorname{id}_{R} \otimes \varphi_{j}: R \otimes_{R_{j+1}} M_{j+1} \rightarrow R \otimes_{R_{j}} M_{j}$ $(0 \leq j<l)$. Then we define $\nabla^{(k)}$ to be the composition

$$
\nabla^{(k)}: M \stackrel{\chi_{l}^{-1}}{\longrightarrow} R \otimes_{R_{l}} M_{l} \stackrel{\mathrm{d}_{R}^{(k)} \otimes \operatorname{id}_{M_{l}}}{\longrightarrow}\left(\hat{\Omega}_{R / K}\right)_{k} \otimes_{R_{l}} M_{l} \stackrel{\mathrm{id}_{\hat{\Omega}} \otimes \chi_{l}}{\longrightarrow}\left(\hat{\Omega}_{R / K}\right)_{k} \otimes_{R} M .
$$

This is well defined, because $\mathrm{d}_{R}^{(k)}$ is $R_{l}$-linear, and is also independent of the chosen $l$. The definition also shows that the necessary conditions for $\nabla$ being an integrable iterative connection are fulfilled modulo degrees $\geq p^{l}$, since $\mathrm{d}_{R}$ is an integrable iterative connection. As $l$ can be chosen arbitrarily large, $\nabla$ fulfills all conditions for being an integrable iterative connection.

It remains to show that $\bigcap_{0<j<p^{l}} \operatorname{Ker}\left(\nabla^{(j)}\right)=\left(\varphi_{0} \circ \cdots \circ \varphi_{l-1}\right)\left(M_{l}\right)$. Since we have just constructed an iterative connection on $M$, by Corollary 4.5, $M$ is projective and, by the same argument, all $M_{l}$ are projective. Hence $\operatorname{Ker}\left(\mathrm{d}_{R}^{(j)} \otimes \operatorname{id}_{M_{l}}\right)=$ $\operatorname{Ker}\left(\mathrm{d}_{R}^{(j)}\right) \otimes_{R_{l}} M_{l}$ for all $j<p^{l}$, and so

$$
\bigcap_{0<j<p^{l}} \operatorname{Ker}\left(\nabla^{(j)}\right)=\chi_{l}\left(R_{l} \otimes_{R_{l}} M_{l}\right)=\left(\varphi_{0} \circ \cdots \circ \varphi_{l-1}\right)\left(M_{l}\right) .
$$

Finally, let $\left(\alpha_{l}\right)_{l \in \mathbb{N}}:\left(M_{l}, \varphi_{l}\right) \rightarrow\left(M_{l}^{\prime}, \varphi_{l}^{\prime}\right)$ be a morphism of Fc-projective systems over $R$. We have to show that $\nabla^{\prime} \circ \alpha_{0}=\left(\operatorname{id}_{\hat{\Omega}} \otimes \alpha_{0}\right) \circ \nabla$ or, equivalently, that for all $k \in \mathbb{N}$

$$
\nabla^{\prime(k)} \circ \alpha_{0}=\left(\operatorname{id}_{\hat{\Omega}} \otimes \alpha_{0}\right) \circ \nabla^{(k)} .
$$

But the last condition is easily seen to hold by choosing $l \in \mathbb{N}$ such that $p^{l}>k$ and by using the definition of the iterative connections above.

In what follows, we will show that the converse is also true, i.e. that a module with integrable iterative connection gives rise to an Fc-projective system over $R$. For this, we consider the quotient field $F:=\operatorname{Quot}(R)$ of $R$ and a monomial ordering on $\hat{\Omega}_{F / K}=F\left[\left[\mathrm{~d}^{(i)} t_{j}\right]\right]$, namely the lexicographical order, where the variables are ordered by d ${ }^{\left(i_{1}\right)} t_{j_{1}}>\mathrm{d}^{\left(i_{2}\right)} t_{j_{2}}$ if $i_{1}>i_{2}$ or if $i_{1}=i_{2}$ and $j_{1}>j_{2}$. The leading term of $\omega \in \hat{\Omega}_{F / K}$ (if it exists) is then denoted by $\operatorname{LT}(\omega)$. 
Lemma 8.5. Let $\omega \in \hat{\Omega}_{F / K}$ be homogeneous of degree $p^{l}$ and $\omega \notin F \hat{\Omega}_{F / K}^{p^{l}}$. Let $\mathrm{d}^{\left(i_{0}\right)} t_{j_{0}}$ be the highest variable with the property that there exist $e_{0} \in \mathbb{N}, p \nmid e_{0}$ and a monomial $\omega^{\prime} \in \hat{\Omega}_{F / K}$ such that $\left(\mathrm{d}^{\left(i_{0}\right)} t_{j_{0}}\right)^{e_{0}} \omega^{\prime}$ is a monomial term of $\omega$. Let $e_{0}$ and $\omega^{\prime}$ be chosen such that $\left(\mathrm{d}^{\left(i_{0}\right)} t_{j_{0}}\right)^{e_{0}} \omega^{\prime}$ is maximal among those monomials. Then for every $k \leq p^{l}(p-1)$, we have

$$
\operatorname{LT}\left(\mathrm{d}_{\hat{\Omega}}^{(k)}(\omega)\right) \leq e_{0} \mathrm{~d}^{\left(i_{0}+p^{l}(p-1)\right)} t_{j_{0}} \cdot\left(\mathrm{d}^{\left(i_{0}\right)} t_{j_{0}}\right)^{e_{0}-1} \omega^{\prime},
$$

with equality if and only if $k=p^{l}(p-1)$ and $i_{0}<p^{l}$.

Proof. For $i \in \mathbb{N}, j \in\{1, \ldots, m\}, e \in \mathbb{N}_{+}$and $k \in \mathbb{N}$, we have

$$
\mathrm{d}_{\hat{\Omega}}^{(k)}\left(\left(\mathrm{d}^{(i)} t_{j}\right)^{e}\right)=\sum_{k_{1}+\cdots+k_{e}=k}\left(\begin{array}{c}
i+k_{1} \\
i
\end{array}\right) \cdots\left(\begin{array}{c}
i+k_{e} \\
i
\end{array}\right) \mathrm{d}^{\left(i+k_{1}\right)} t_{j} \cdots \mathrm{d}^{\left(i+k_{e}\right)} t_{j} .
$$

So

$$
\begin{aligned}
\operatorname{LT}\left(\mathrm{d}_{\hat{\Omega}}^{(k)}\left(\left(\mathrm{d}^{(i)} t_{j}\right)^{e}\right)\right) & =e \cdot\left(\begin{array}{c}
i+k \\
i
\end{array}\right) \mathrm{d}^{(i+k)} t_{j}\left(\mathrm{~d}^{(i)} t_{j}\right)^{e-1} \quad \text { if } e\left(\begin{array}{c}
i+k \\
i
\end{array}\right) \neq 0 \in \mathbb{F}_{p}, \\
\mathrm{~d}_{\hat{\Omega}}^{(k)}\left(\left(\mathrm{d}^{(i)} t_{j}\right)^{e}\right) & =0 \quad \text { if } p \mid e \text { and } p \nmid k \text { and } \\
\mathrm{d}_{\hat{\Omega}}^{(k)}\left(\left(\mathrm{d}^{(i)} t_{j}\right)^{e}\right) & =\left(\mathrm{d}_{\hat{\Omega}}^{\left(\frac{k}{p}\right)}\left(\left(\mathrm{d}^{(i)} t_{j}\right)^{\frac{e}{p}}\right)\right)^{p} \text { if } p \mid e \text { and } p \mid k .
\end{aligned}
$$

So for $k \leq p^{l}(p-1)$, a variable $\mathrm{d}^{(i)} t_{j} \neq \mathrm{d}^{\left(i_{0}\right)} t_{j_{0}}$ occurring in $\omega$ gives a contribution to $\mathrm{d}_{\hat{\Omega}}^{(k)}(\omega)$ of variables

(i) less than $\mathrm{d}^{\left(i_{0}+k\right)} t_{j_{0}}$ if it occurs in a power not divided by $p$ and

(ii) less than $\mathrm{d}^{\left(i+\frac{k}{p}\right)} t_{j}$ otherwise.

In the second case, $i \leq p^{l-1}$, since $\omega$ is of degree $p^{l}$, and so $i+\frac{k}{p} \leq p^{l-1}+p^{l-1}(p-1)=$ $p^{l}$. So $\mathrm{d}^{\left(i+\frac{k}{p}\right)} t_{j}<\mathrm{d}^{\left(i_{0}+p^{l}\right)} t_{j_{0}}$. Therefore the highest variable that may occur is $\mathrm{d}^{\left(i_{0}+k\right)} t_{j_{0}}$ (or $\mathrm{d}^{\left(i_{0}+p^{l}\right)} t_{j_{0}}$ if $k<p^{l}$ ), and $\mathrm{d}^{\left(i_{0}+p^{l}(p-1)\right)} t_{j_{0}}$ occurs if and only if $k=p^{l}(p-1)$ and $\left(\begin{array}{c}i_{0}+p^{l}(p-1) \\ i_{0}\end{array}\right) \neq 0 \in \mathbb{F}_{p}$, i.e. $i_{0} \neq p^{l}$.

The highest corresponding monomial then is $e_{0} \mathrm{~d}^{\left(i_{0}+p^{l}(p-1)\right)} t_{j_{0}} \cdot\left(\mathrm{d}^{\left(i_{0}\right)} t_{j_{0}}\right)^{e_{0}-1} \omega^{\prime}$.

Proposition 8.6. Every $R$-module $M$ with integrable iterative connection $\nabla$ defines an Fc-projective system $\left(M_{l}, \varphi_{l}\right)$ over $R$, where $M_{l}:=\bigcap_{0<j<p^{l}} \operatorname{Ker}\left(\nabla^{(j)}\right)$ and $\varphi_{l}$ : $M_{l+1} \rightarrow M_{l}$ is the inclusion map, and a morphism $f:(M, \nabla) \rightarrow\left(M^{\prime}, \nabla^{\prime}\right)$ of modules with higher connection defines a morphism $\alpha:\left(M_{l}, \varphi_{l}\right) \rightarrow\left(M_{l}^{\prime}, \varphi_{l}^{\prime}\right)$ of Fc-projective systems over $R$ by $\alpha_{l}:=\left.f\right|_{M_{l}}$.

Proof. Since $\nabla$ is an integrable iterative connection on $M, \nabla^{(1)}$ is an integrable connection on $M$ (cf. proof of Proposition 7.1 (iii)), and is of $p$-curvature zero. Now let $M_{1}:=\operatorname{Ker}\left(\nabla^{(1)}\right)\left(=\bigcap_{0<j<p^{1}} \operatorname{Ker}\left(\nabla^{(j)}\right)\right.$, since $\nabla$ is iterative). Then by Cartier's Theorem on the $p$-curvature (cf. Kat70, Thm. 5.1), $M_{1}$ is an $R_{1}$-module and $R \otimes_{R_{1}} M_{1} \rightarrow M$ is an isomorphism of $R$-modules.

Next, we will show that $\nabla\left(M_{1}\right) \subset\left(\hat{\Omega}_{R / K}\right)^{p} \otimes_{R_{1}} M_{1}$. Since $\left(\hat{\Omega}_{R / K}\right)^{p}$ is isomorphic to $\hat{\Omega}_{R_{1} / K}$ as an algebra by the map $\left(\mathrm{d}^{(i)} x\right)^{p} \mapsto \mathrm{d}^{(i)}\left(x^{p}\right)$, this means that essentially 
$\left.\nabla\right|_{M_{1}}$ is an integrable iterative connection on the $R_{1}$-module $M_{1}$. It then follows inductively that $R_{l} \otimes_{R_{l+1}} M_{l+1} \cong M_{l}$ and that, essentially, $\left.\nabla\right|_{M_{l+1}}$ is an integrable iterative connection on the $R_{l+1}$-module $M_{l+1}$.

Since $M_{1}$ and $\hat{\Omega}_{R / K}$ are locally free, and hence localisation is injective, it suffices to show the statement for the quotient field $F:=\operatorname{Quot}(R)$ of $R$. For simplicity, we again write $M$ and $M_{1}$ for what should be $F \otimes_{R} M$ and $F_{1} \otimes_{R_{1}} M_{1}$ :

Since $\nabla$ is iterative, we only have to show that $\nabla^{\left(p^{l}\right)}\left(M_{1}\right) \subset\left(\hat{\Omega}_{F / K}\right)^{p} \otimes_{F_{1}} M_{1}$ for all $l \geq 1$. So fix an $F_{1}$-basis $\boldsymbol{b}=\left(b_{1}, \ldots, b_{n}\right)$ of $M_{1}$ (written as a row) and let $A_{l} \in$ $\operatorname{Mat}_{n}\left(\hat{\Omega}_{p^{l}}\right)$ with $\nabla^{\left(p^{l}\right)}(\boldsymbol{b})=\boldsymbol{b} A_{l}$ 约 From $0={ }_{\Omega^{\prime}} \nabla^{\left(p^{l}\right)}\left(\nabla^{(1)}(\boldsymbol{b})\right)={ }_{\hat{\Omega}} \nabla^{(1)}\left(\nabla^{\left(p^{l}\right)}(\boldsymbol{b})\right)=$ $\boldsymbol{b} \mathrm{d}_{\hat{\Omega}}^{(1)}\left(A_{l}\right)$ we conclude $\mathrm{d}_{\hat{\Omega}}^{(1)}\left(A_{l}\right)=0$. Assume there is an entry

$$
\omega \in \hat{\Omega}_{p^{l}} \subset F\left[\mathrm{~d}^{(i)} t_{j} \mid i=1, \ldots, p^{l}, j=1, \ldots, m\right]
$$

of $A_{l}$ with $\mathrm{LT}(\omega)=r \mathrm{~d}^{\left(p^{l}\right)} t_{j}$ (for some $r \in F$ and $j \in\{1, \ldots, m\}$ ). Since $\mathrm{d}_{\hat{\Omega}}^{(1)}\left(r \mathrm{~d}^{\left(p^{l}\right)} t_{j}\right)=\mathrm{d}^{(1)}(r) \mathrm{d}^{\left(p^{l}\right)} t_{j}+r \mathrm{~d}^{\left(p^{l}+1\right)} t_{j}$ and since for all other monomials of $\omega$ the image under $\mathrm{d}_{\hat{\Omega}}^{(1)}$ doesn't contain the variable $\mathrm{d}^{\left(p^{l}+1\right)} t_{j}$, we obtain $\mathrm{d}_{\hat{\Omega}}^{(1)}(\omega) \neq 0$, a contradiction. So

$$
\omega \in F\left[\mathrm{~d}^{(i)} t_{j} \mid i=1, \ldots, p^{l}-1, j=1, \ldots, m\right] .
$$

Furthermore, since $\nabla$ is iterative, ${ }_{\Omega} \nabla^{\left(p^{l}(p-1)\right)} \circ \nabla^{\left(p^{l}\right)}=\left(\begin{array}{c}p^{l+1} \\ p^{l}\end{array}\right) \nabla^{\left(p^{l+1}\right)}=0$, and therefore

$$
0={ }_{\hat{\Omega}} \nabla^{\left(p^{l}(p-1)\right)}\left(\boldsymbol{b} A_{l}\right)=\boldsymbol{b} \cdot \mathrm{d}_{\hat{\Omega}}^{\left(p^{l}(p-1)\right)}\left(A_{l}\right)+\sum_{k=0}^{p^{l}(p-1)-1} \nabla^{\left(p^{l}(p-1)-k\right)}(\boldsymbol{b}) \cdot \mathrm{d}_{\hat{\Omega}}^{(k)}\left(A_{l}\right) .
$$

If $A_{l} \notin \operatorname{Mat}_{n}\left(F \hat{\Omega}^{p}\right)$, then by the previous lemma, $\mathrm{d}^{\left(p^{l}(p-1)\right)}\left(A_{l}\right)$ has an entry with leading term $e_{0} \mathrm{~d}^{\left(i_{0}+p^{l}(p-1)\right)} t_{j_{0}}\left(\mathrm{~d}^{\left(i_{0}\right)} t_{j_{0}}\right)^{e_{0}-1} \cdot \omega^{\prime}$ for some $\omega^{\prime} \in \hat{\Omega}, i_{0} \leq p^{l}$ and $j_{0} \in\{1, \ldots, m\}$, and the variables occurring in $\mathrm{d}_{\hat{\Omega}}^{(k)}\left(A_{l}\right)\left(k<p^{l}(p-1)-1\right)$ are less than $\mathrm{d}^{\left(i_{0}+p^{l}(p-1)\right)} t_{j_{0}}$. Moreover, those occurring in $\nabla^{\left(p^{l}(p-1)-k\right)}(\boldsymbol{b})$ are even less than or equal to $\mathrm{d}^{\left(p^{l}(p-1)\right)} t_{m}$. So we would have ${ }_{\hat{\Omega}} \nabla^{\left(p^{l}(p-1)\right)}\left(\boldsymbol{b} A_{l}\right) \neq 0$. Therefore $A_{l} \in \operatorname{Mat}_{n}\left(F \hat{\Omega}^{p}\right)$.

At last, since $\mathrm{d}_{\hat{\Omega}}^{(1)}\left(A_{l}\right)=0$, in fact $A_{l} \in \operatorname{Mat}_{n}\left(\hat{\Omega}^{p}\right)$, which completes the proof.

Theorem 8.7. The category $\mathbf{P r o j}_{R}$ of $F c$-projective systems over $R$ and the category $\mathbf{I C o n}_{\mathbf{i n t}}(R / K)$ are equivalent. Furthermore, if $R$ is an algebraic function field in one variable over $K$ and $\phi \in \operatorname{ID}_{K}(R)$ with $\phi^{(1)} \neq 0$, then they are also equivalent to the category $\mathbf{I D}_{R}$ of iterative differential modules over $(R, \phi)$ (cf. Mat01, Ch. 2 and $\mathrm{MvdP03}$, Ch. 2) and to the category $\operatorname{ICon}(R / K)$.

Proof. The first statement follows immediately from the previous two propositions, since the given maps are functors that are inverses to each other. The proof of Proposition 8.6] shows that the integrability condition is not necessary when $R$ is

\footnotetext{
${ }^{3}$ For simplicity we use vector notation: $\boldsymbol{b} A_{l}$ denotes the row vector with $j$-th component $\sum_{i=1}^{n}\left(A_{l}\right)_{i j} b_{i}$, and $\nabla$ and $\mathrm{d}_{\hat{\Omega}}$ are always applied to the components of a vector or a matrix. Also, we abbreviate $\hat{\Omega}_{F / K}$ by $\hat{\Omega}$.
} 
an algebraic function field in one variable. So $\operatorname{ICon}(R / K)$ is equivalent to $\operatorname{Proj}_{R}$ in this case. Furthermore, Matzat and van der Put showed in Mat01, Thm. 2.8, resp. MvdP03, Prop. 5.1, that $\mathbf{I D}_{R}$ is also equivalent to $\mathbf{P r o j}_{R}$.

Corollary 8.8. If $K$ is algebraically closed and $R$ is smooth over $K$, then the category $\mathbf{I C o n}_{\mathbf{i n t}}(R / K)$ is equivalent to the category of stratified modules over $R$.

Proof. By the previous theorem, the category $\operatorname{ICon}_{\mathbf{i n t}}(R / K)$ is equivalent to the category $\operatorname{Proj}_{R}$ of Fc-projective systems over $R$. Furthermore, under the given assumptions, $\operatorname{Proj}_{R}$ is equivalent to the category of stratified modules over $R$, by Gie75, Thm. 1.3. So the statement follows.

In the previous section, we have seen that the same corollary holds for $\operatorname{char}(K)=$ 0 (cf. Corollary 7.3). However, there is still no proof of this equivalence that works in arbitrary characteristic. Furthermore, it is an open question whether stratifications and integrable iterative connections are equivalent or even related, when $R$ is not smooth over $K$.

\section{Higher Connections on SCHEMES}

Next, we outline a generalisation of modules with iterative connections to modules over schemes.

Throughout this section, let $K$ be a perfect field, let $X$ be a nonsingular, geometrically integral $K$-scheme which is separated and of finite type over $K$, and let $\mathcal{O}_{X}$ denote the structure sheaf of $X$.

Definition 9.1. We define the sheaf of higher differentials on $X$, denoted by $\hat{\Omega}_{X / K}$, to be the sheaf associated to the presheaf given by

$$
U \mapsto \hat{\Omega}_{\mathcal{O}_{X}(U) / K}
$$

for each open subset $U \subseteq X$ and by the restriction maps

$$
D\left(\rho_{V}^{U}\right): \hat{\Omega}_{\mathcal{O}_{X}(U) / K} \rightarrow \hat{\Omega}_{\mathcal{O}_{X}(V) / K}
$$

for all open subsets $V \subseteq U \subseteq X$, as defined in Proposition 3.12, where $\rho_{V}^{U}$ : $\mathcal{O}_{X}(U) \rightarrow \mathcal{O}_{X}(V)$ is the restriction map of $\mathcal{O}_{X}$.

Remark 9.2. By Proposition 3.12, for all open subsets $V \subseteq U \subseteq X$, the diagram

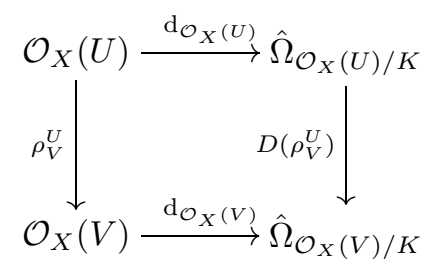

commutes and so the collection of maps $\mathrm{d}_{\mathcal{O}_{X}(U)}$ induces a morphism of sheaves of $K$-algebras $\mathrm{d}_{X}: \mathcal{O}_{X} \rightarrow \hat{\Omega}_{X / K}$.

Proposition 9.3. If $X$ is an affine scheme, then the presheaf $U \mapsto \hat{\Omega}_{\mathcal{O}_{X}(U) / K}$ is in fact a sheaf. 
Proof. The given presheaf is a sheaf if and only if for all open subsets $U \subseteq X$ and all open coverings $\bigcup_{i \in I} U_{i}=U$, the sequence

$$
0 \rightarrow \hat{\Omega}_{\mathcal{O}_{X}(U) / K} \rightarrow \prod_{i \in I} \hat{\Omega}_{\mathcal{O}_{X}\left(U_{i}\right) / K} \rightarrow \prod_{i, j \in I} \hat{\Omega}_{\mathcal{O}_{X}\left(U_{i} \cap U_{j}\right) / K}
$$

is exact. Since this is a sequence of cgas, it suffices to show that the sequence is exact in each homogeneous component.

For every open subset $V \subseteq U, \mathcal{O}_{X}(V)$ is a localisation of $\mathcal{O}_{X}(U)$, and so by Proposition 3.12, $\hat{\Omega}_{\mathcal{O}_{X}(V) / K} \cong \mathcal{O}_{X}(V) \otimes \hat{\Omega}_{\mathcal{O}_{X}(U) / K}$. By Corollary 3.15, the homogeneous components $\left(\hat{\Omega}_{\mathcal{O}_{X}(U) / K}\right)_{k}(k \in \mathbb{N})$ are projective $\mathcal{O}_{X}(U)$-modules, and therefore tensoring with $\left(\hat{\Omega}_{\mathcal{O}_{X}(U) / K}\right)_{k}$ is exact. So the sequence above is exact in each homogeneous component if the sequence

$$
0 \rightarrow \mathcal{O}_{X}(U) \rightarrow \prod_{i \in I} \mathcal{O}_{X}\left(U_{i}\right) \rightarrow \prod_{i, j \in I} \mathcal{O}_{X}\left(U_{i} \cap U_{j}\right)
$$

is exact. But this is true since $\mathcal{O}_{X}$ is itself a sheaf.

As an immediate consequence of this proposition, we have the following corollary:

Corollary 9.4. For every affine open subset $U \subseteq X$, we have $\hat{\Omega}_{X / K}(U)=\hat{\Omega}_{\mathcal{O}_{X}(U) / K}$.

Definition 9.5. Let $M$ be a coherent $\mathcal{O}_{X}$-module. A higher connection on $M$ is a morphism of sheaves $\nabla: M \rightarrow \hat{\Omega}_{X / K} \otimes_{\mathcal{O}_{X}} M$ which locally (i.e. on affine open subsets) is a higher connection in the sense of Section 4. The higher connection $\nabla$ is called iterative, resp. integrable iterative, if $\nabla$ locally is an iterative, resp. integrable iterative, connection.

Remark 9.6. (1) By Corollary 4.5, every coherent $\mathcal{O}_{X}$-module $M$ that admits a higher connection $\nabla: M \rightarrow \hat{\Omega}_{X / K} \otimes_{\mathcal{O}_{X}} M$ is locally free and of finite rank.

(2) Following the notion of modules with higher connection over rings, the categories of coherent $\mathcal{O}_{X}$-modules with higher connection, with iterative connection and with integrable iterative connection will be denoted by $\operatorname{HCon}(X / K)$ and $\mathbf{I C o n}(X / K)$, resp. $\mathbf{I C o n}_{\text {int }}(X / K)$. By standard methods of algebraic geometry, one obtains that again $\operatorname{HCon}(X / K)$, $\operatorname{ICon}(X / K)$ and $\operatorname{ICon}_{\text {int }}(X / K)$ are tensor categories over $K$ and that they are Tannakian categories. And if $X$ has a $K$-rational point, they are in fact neutral Tannakian categories over $K$.

\section{PicARD-Vessiot theORY}

In Section [6] we showed that the category of modules with higher connection $\operatorname{HCon}(R / K)$ with fibre functor $\boldsymbol{\omega}_{K}: \operatorname{HCon}(R / K) \rightarrow \operatorname{Vect}(K)$ is a neutral Tannakian category over $K$ and that $\mathbf{I C o n}(R / K)$ and $\mathbf{I C o n}_{\text {int }}(R / K)$ are Tannakian subcategories. By Tannaka duality, this means that $\operatorname{HCon}(R / K)$ is equivalent to the category of finite dimensional representations of a certain group scheme and that $\mathbf{I C o n}(R / K)$ and $\mathbf{I C o n}_{\mathbf{i n t}}(R / K)$ are equivalent to the category of finite dimensional representations of quotients of this group scheme. (In positive characteristic, the group scheme associated to $\operatorname{ICon}_{\text {int }}(R / K)$ is isomorphic to the fundamental group scheme for F-divided sheaves $\Pi^{F d i v}\left(\operatorname{Spec}(R), \boldsymbol{\omega}_{K}\right)$ in [San07] and by Theorem 8.7 and Remark 8.3.) Furthermore, for every module with higher (or iterative 
or integrable iterative) connection $(M, \nabla)$, one obtains the Tannakian Galois group $G_{(M, \nabla)}$, which is the group scheme corresponding to the smallest Tannakian subcategory that contains $(M, \nabla)$. In this section, we obtain these Galois group schemes for modules with iterative connection from another point of view, namely as automorphisms of solution rings (so-called pseudo-Picard-Vessiot rings, or PPV-rings for short). The fact that the automorphism group scheme of a PPV-ring of $(M, \nabla)$ is isomorphic to the Tannakian Galois group scheme $G_{(M, \nabla)}$ can be shown in the same manner as in vdPS03, Thm. 2.33, for differential modules, or as in Pap08, Sections $3.5-4.5$, for t-motives, and is sketched in Remark 10.13 at the end of this section.

Some of the constructions and proofs given here will be quite similar to those of T. Dyckerhoff in Dyc08, who used Galois group schemes for obtaining a differential Galois theory in characteristic zero over nonalgebraically closed fields of constants. However, we have to deal with an additional phenomenon occurring in positive characteristic, namely inseparability of the extensions and nonreduced group schemes.

Since the Picard-Vessiot theory that we provide does not only work for modules with iterative connections, but for a large class of higher derivations, we make the following definition.

Definition 10.1. Let $F$ be a $K$-algebra and let $\tilde{\Omega}$ be an $F$-cga. A higher derivation $\theta: F \rightarrow \tilde{\Omega}$ will be called iterable if the following hold:

(i) For all $k \in \mathbb{N}$ the homogeneous component $\tilde{\Omega}_{k}$ is generated by $\left\{\theta^{(k)}(r) \mid\right.$ $r \in F\}$.

(ii) $\theta$ can be extended to a continuous endomorphism $\theta_{\tilde{\Omega}}: \tilde{\Omega} \rightarrow \tilde{\Omega}$, satisfying the iteration rule $\theta_{\tilde{\Omega}}^{(i)} \circ \theta_{\tilde{\Omega}}^{(j)}=\left(\begin{array}{c}i+j \\ i\end{array}\right) \theta_{\tilde{\Omega}}^{(i+j)}(i, j \in \mathbb{N})$, or equivalently satisfying $\left(a \cdot \theta_{\tilde{\Omega}}\right) \circ\left(b \cdot \theta_{\tilde{\Omega}}\right)=(a+b) \cdot \theta_{\tilde{\Omega}}$ for all $a, b \in K^{\mathrm{sep}}$.

Let $\theta$ be iterable, $M$ be an $F$-module and $\Theta$ be a higher $\theta$-derivation on $M$. As for higher connections we can define an endomorphism $\Theta_{\tilde{\Omega}}$ on $\tilde{\Omega} \otimes_{F} M$ by

$$
\Theta_{\tilde{\Omega}}(\omega \otimes x):=\theta_{\tilde{\Omega}}(\omega) \cdot \Theta(x)
$$

for all $\omega \in \tilde{\Omega}$ and $x \in M$. The $\theta$-derivation $\Theta$ is called iterable if $\Theta_{\tilde{\Omega}}$ satisfies the iteration rule $\Theta_{\tilde{\Omega}}^{(i)} \circ \Theta_{\tilde{\Omega}}^{(j)}=\left(\begin{array}{c}i+j \\ i\end{array}\right) \Theta_{\tilde{\Omega}}^{(i+j)}(i, j \in \mathbb{N})$.

Example 10.2. The universal derivation $\mathrm{d}_{F}: F \rightarrow \hat{\Omega}_{F / K}$ is an iterable higher derivation with extension $\mathrm{d}_{\hat{\Omega}}$. Other examples are appropriate extensions of the universal derivation to extensions of $F$ (e.g. to PPV-rings $R$ over $F$ for some iterable higher differential equation; cf. Definition 10.5) or the canonical extension of $\mathrm{d}_{K(t)}$ to $K((t))$ (i.e. a higher derivation $\theta: K((t)) \rightarrow K((t)) \otimes_{K(t)} \hat{\Omega}_{K(t) / K}$ ).

Further examples are iterative derivations $\phi: F \rightarrow F[[T]]$ with $\phi^{(1)} \neq 0$ (the additional assumption is only necessary to fulfill condition (i)) and also $m$-variate iterative derivations $\phi: F \rightarrow F\left[\left[T_{1}, \ldots, T_{m}\right]\right]$ defined by F. Heiderich in his Diplomarbeit (cf. Hei07).

Let $\theta_{F}: F \rightarrow \tilde{\Omega}$ be an iterable higher derivation and let $L / F$ be a finite field extension. If $L / F$ is separable, then $\theta_{F}$ extends uniquely to a higher derivation $\theta_{L}: L \rightarrow L \otimes \tilde{\Omega}$ (cf. Proposition 3.7 and Example 3.5), which therefore is also iterable. If $L / F$ is not separable, there may not exist an extension of $\theta_{F}$ to $L$. However, if $\tilde{\Omega}$ has no nilpotent elements, there exists at most one extension, which 
then is iterable. This relies on the fact that for some $k \geq 0, L^{p^{k}}$ lies in a separable algebraic extension $\tilde{F}$ of $F$, and hence $\theta_{L}(s)^{p^{k}}=\theta_{\tilde{F}}\left(s^{p^{k}}\right)$ determines $\theta_{L}(s)$ uniquely for all $s \in L$.

Remark 10.3. If a higher derivation $\theta: F \rightarrow \tilde{\Omega}$ is iterable, the extension $\theta_{\tilde{\Omega}}$ is unique, since $\tilde{\Omega}_{k}$ is generated by $\theta^{(k)}(F)$ for all $k$. Furthermore, the iteration rule implies that $\theta_{\tilde{\Omega}}$ is an automorphism of $\tilde{\Omega}$.

Notation. From now on, we fix an arbitrary field $K$, a field $F$ containing $K$, an $F$-cga $\tilde{\Omega}$ having no zero-divisors, and an iterable higher derivation $\theta: F \rightarrow \tilde{\Omega}$, such that $K=\{t \in F \mid \theta(t)=t\}$.

Definition 10.4. A $\theta$-ring is an $F$-algebra $R$ together with an iterable higher derivation $\theta_{R}: R \rightarrow R \otimes \tilde{\Omega}$ that extends $\theta$. The pair $\left(R, \theta_{R}\right)$ is called a $\theta$-field if $R$ is a field. The set $C_{R}:=\left\{r \in R \mid \theta_{R}(r)=r \otimes 1\right\}$ is called the ring of constants of $\left(R, \theta_{R}\right)$. An ideal $I \unlhd R$ is called a $\theta$-ideal if for all $k \in \mathbb{N}, \theta_{R}^{(k)}(I) \subseteq I \otimes \tilde{\Omega}_{k}$; $R$ is $\theta$-simple if $R$ has no proper nontrivial $\theta$-ideals. Localisations of $\theta$-rings are again $\theta$-rings by $\theta\left(\frac{r}{s}\right):=\theta(r) \theta(s)^{-1}$ (as for iterative derivations one easily shows that these extensions are again iterable). The tensor product $R \otimes_{F} \tilde{R}$ of two $\theta$-rings $R$ and $\tilde{R}$ is a $\theta$-ring by

$$
\theta_{R \otimes \tilde{R}}^{(k)}(r \otimes \tilde{r}):=\sum_{i+j=k} \theta_{R}^{(i)}(r) \cdot \theta_{\tilde{R}}^{(j)}(\tilde{r}) \in R \otimes \tilde{R} \otimes \tilde{\Omega},
$$

for all $k \geq 0, r \in R$ and $\tilde{r} \in \tilde{R}$. A homomorphism of $\theta$-rings $f: R \rightarrow \tilde{R}$ is called a $\theta$-homomorphism if $\theta_{\tilde{R}} \circ f=\left(f \otimes \mathrm{id}_{\tilde{\Omega}}\right) \circ \theta_{R}$. The set of all $\theta$-homomorphisms is denoted by $\operatorname{Hom}^{\theta}(R, \tilde{R})$. Furthermore, for $\theta$-rings $R \geq \tilde{R} \geq F$, the set of $\theta$ automorphisms of $R$ that leave the elements of $\tilde{R}$ fixed is denoted by $\operatorname{Aut}^{\theta}(R / \tilde{R})$.

Given a $\theta$-ring $R$ and a $K$-algebra $L$, the tensor product $R \otimes_{K} L$ can be given the structure of a $\theta$-ring by $\theta_{R \otimes_{K} L}(r \otimes a)=\theta_{R}(r) \otimes a(r \in R, a \in L)$. We say that $\theta_{R}$ is extended trivially to $R \otimes_{K} L$.

Let $A=\sum_{k=0}^{\infty} A_{k} \in \mathrm{GL}_{n}(\tilde{\Omega})$ with $A_{0}=\mathbf{1}_{n}$ (identity matrix) and for all $k, l \in \mathbb{N}$, $\left.\left(\begin{array}{c}k+l \\ l\end{array}\right) A_{k+l}=\sum_{i+j=l} \theta^{(i)}\left(A_{k}\right) \cdot A_{j} \in \operatorname{Mat}(n \times n), \tilde{\Omega}_{k+l}\right)$. Then an equation

$$
\theta(\boldsymbol{y})=A \boldsymbol{y},
$$

where $\boldsymbol{y}$ is a vector of indeterminates, is called an iterable higher differential equation.

Notice that the condition on the matrices $A_{k}$ is the same as saying that for an $F$-vector space $M$ with basis $\boldsymbol{b}=\left(b_{1}, \ldots, b_{n}\right)$, the $\theta$-derivation $\Theta$ defined by $\Theta(\boldsymbol{b})=\boldsymbol{b} A^{-1}$ is iterable, and a vector $\boldsymbol{x} \in F^{n}$ is a solution of the equation (*) if and only if $\boldsymbol{b} \boldsymbol{x} \in M$ is constant, i.e. satisfies $\Theta(\boldsymbol{b} \boldsymbol{x})=\boldsymbol{b} \boldsymbol{x}$.

Definition 10.5. A $\theta$-ring $\left(R, \theta_{R}\right)$ is called a pseudo-Picard-Vessiot ring (PPVring) for $\theta(\boldsymbol{y})=A \boldsymbol{y}$, if the following holds:

(1) $R$ is $\theta$-simple.

(2) There is a fundamental solution matrix $Y \in \mathrm{GL}_{n}(R)$, i.e. an invertible matrix satisfying $\theta_{R}(Y)=A Y$.

(3) As an $F$-algebra, $R$ is generated by the coefficients of $Y$ and by $\operatorname{det}(Y)^{-1}$.

(4) $C_{R}=C_{F}=K$

The quotient field $E=\operatorname{Quot}(R)$ is called a pseudo-PV-field for the equation (*). 
Remark 10.6. Analogous to Mat01, Prop. 3.2, resp. MvdP03, Lemma 3.2, one shows that $R$ is an integral domain, so the quotient field $E$ exists. Furthermore, for every $\theta$-simple $\theta$-ring which is finitely generated as an $F$-algebra, its constants are algebraic over $K$. Hence if $K$ is algebraically closed, a PPV-ring for the equation * is given by $R=S / P$, where $S:=F\left[X_{i j}, \operatorname{det}(X)^{-1} \mid i, j=1, \ldots, n\right]$ is a $\theta$-ring by $\theta_{S}(X):=A X$ and $P \unlhd S$ is a maximal $\theta$-ideal. Hence in this case PPV-rings always exist and, by a similar proof as for Mat01, Thm. 3.4, are unique up to $\theta$-isomorphisms.

For a PPV-ring $R / F$ we define the functor

$$
\underline{\operatorname{Aut}}^{\theta}(R / F):(\text { Algebras } / K) \rightarrow(\text { Groups }), L \mapsto \operatorname{Aut}^{\theta}\left(R_{L} / F_{L}\right),
$$

where $F_{L}:=F \otimes_{K} L, R_{L}:=R \otimes_{K} L$ and $\theta$, resp. $\theta_{R}$, is extended trivially to $F_{L}$, resp. $R_{L}$.

We will show that the functor $\operatorname{Aut}^{\theta}(R / F)$ is representable by a $K$-algebra of finite type and hence is an affine group scheme of finite type over $K$.

Lemma 10.7. Let $R$ be a $\theta$-simple $\theta$-ring with $C_{R}=K$, let $L$ be a finitely generated $K$-algebra and let $R_{L}:=R \otimes_{K} L$, with $\theta$-structure trivially extended from $R$. Then there is a bijection

$$
\begin{aligned}
\mathcal{I}(L) & \longleftrightarrow \mathcal{I}^{\theta}\left(R_{L}\right), \\
I & \longmapsto R_{L}\left(1 \otimes_{K} I\right)=R \otimes_{K} I, \\
J \cap\left(1 \otimes_{K} L\right) & \longleftrightarrow J
\end{aligned}
$$

between the ideals of $L$ and the $\theta$-ideals of $R_{L}$.

Proof. Obviously, both maps are well defined, so we only have to show that they are inverses to each other.

(i) We need to show that for $I \in \mathcal{I}(L)$, we have $\left(R \otimes_{K} I\right) \cap\left(1 \otimes_{K} L\right)=I$.

It is clear that $I$ is contained in the left side. For the other inclusion, let $\left\{e_{i} \mid\right.$ $i \in \tilde{N}\}$ be a $K$-basis of $I$. Then $\left(R \otimes_{K} I\right)$ is a free $R$-module with the same basis and an element $f=\sum_{i \in \tilde{N}} r_{i} \otimes e_{i} \in\left(R \otimes_{K} I\right)$ is constant, if and only if all $r_{i}$ are constant, i.e. if $f \in I$.

(ii) We need to show that for $J \in \mathcal{I}_{\theta}\left(R_{L}\right)$, we have $R \otimes_{K}\left(J \cap\left(1 \otimes_{K} L\right)\right)=J$.

It is clear that the left side is contained in $J$, since $J$ is an ideal. For the other inclusion, let $\left\{e_{i} \mid i \in N\right\}$ be a $K$-basis of $L$, where $N$ denotes an index set. Then $\left\{e_{i} \mid i \in N\right\}$ also is a basis for the free $R$-module $R_{L}$.

For any subset $N_{0} \subseteq N$ and $i_{0} \in N_{0}$, let $\mathfrak{A}_{N_{0}, i_{0}} \unlhd R$ denote the ideal of all $r \in R$ such that there exists an element $g=\sum_{j \in N_{0}} s_{j} \otimes e_{j} \in J$ with $s_{i_{0}}=r$. We will show that $\mathfrak{A}_{N_{0}, i_{0}}$ is a $\theta$-ideal of $R$ and so by $\theta$-simplicity of $R$ is equal to (0) or to $R$ :

Let $r \in \mathfrak{A}_{N_{0}, i_{0}}, g=\sum_{j \in N_{0}} s_{j} \otimes e_{j} \in J$ with $s_{i_{0}}=r$ and $k \in \mathbb{N}$. We have to show that $\theta_{R}^{(k)}(r) \in \mathfrak{A}_{N_{0}, i_{0}} \otimes \tilde{\Omega}_{k}$. So let $\left\{\omega_{\alpha}\right\}$ be an $F$-basis of $\tilde{\Omega}_{k}$ and let $g_{\alpha} \in R_{L}$ such that $\theta^{(k)}(g)=\sum_{\alpha} g_{\alpha} \otimes \omega_{\alpha}$. Since $J$ is a $\theta$-ideal, we have $g_{\alpha} \in J$. On the other hand, let $\theta^{(k)}\left(s_{j}\right)=\sum_{\alpha} s_{\alpha, j} \otimes \omega_{\alpha}$ for some $s_{\alpha, j} \in R$. Then

$$
\theta^{(k)}(g)=\sum_{j \in N_{0}} \theta^{(k)}\left(s_{j}\right) \otimes e_{j}=\sum_{j \in N_{0}} \sum_{\alpha} s_{\alpha, j} \otimes e_{j} \otimes \omega_{\alpha} .
$$


So $g_{\alpha}=\sum_{j \in N_{0}} s_{\alpha, j} \otimes e_{j}$, and therefore $s_{\alpha, i_{0}} \in \mathfrak{A}_{N_{0}, i_{0}}$. Hence,

$$
\theta_{R}^{(k)}(r)=\theta_{R}^{(k)}\left(s_{i_{0}}\right)=\sum_{\alpha} s_{\alpha, i_{0}} \otimes \omega_{\alpha} \in \mathfrak{A}_{N_{0}, i_{0}} \otimes \tilde{\Omega}_{k} .
$$

Now, let $N_{0} \subset N$ be a subset, which is minimal for the property that $\mathfrak{A}_{N_{0}, i_{0}} \neq(0)$ for at least one index $i_{0} \in N_{0}$ (minimal in the lattice of subsets). So there exists $f=\sum_{j \in N_{0}} r_{j} \otimes e_{j} \in J$ with $r_{i_{0}}=1$, and by minimality of $N_{0}$, for all $k>0$ we obtain $\theta^{(k)}(f)=\sum_{\substack{j \in N_{0} \\ j \neq i_{0}}} \theta^{(k)}\left(r_{j}\right) \otimes e_{j}=0$. Hence $f \in J \cap\left(1 \otimes_{K} L\right)$.

Now let $g=\sum_{j \in N} s_{j} \otimes e_{j} \in J$ be an arbitrary element, and denote by $N_{1}$ the set of indices $j$ where $s_{j} \neq 0$. By definition, for all $i \in N_{1}, \mathfrak{A}_{N_{1}, i} \neq(0)$. Hence there is $N_{0} \subseteq N_{1}$ minimal as above, $i_{0} \in N_{0}$ and $f=\sum_{j \in N_{0}} r_{j} \otimes e_{j} \in J \cap\left(1 \otimes_{K} L\right)$ with $r_{i_{0}}=1$. By induction on the magnitude of $N_{1}$, we may assume that $g-s_{i_{0}} f \in$ $R \otimes_{K}\left(J \cap\left(1 \otimes_{K} L\right)\right) \subset J$. So $g=\left(g-s_{i_{0}} f\right)+s_{i_{0}} f \in R \otimes_{K}\left(J \cap\left(1 \otimes_{K} L\right)\right)$ and hence $R \otimes_{K}\left(J \cap\left(1 \otimes_{K} L\right)\right)=J$.

Proposition 10.8. Let $R$ be a PPV-ring for the equation (*) and let $T \geq F$ be a $\theta$-simple $\theta$-ring with $C_{T}=K$ such that there exists a fundamental solution matrix $Y \in \mathrm{GL}_{n}(T)$. Then there exists a finitely generated $K$-algebra $U$ and a $T$-linear $\theta$-isomorphism

$$
\gamma_{T}: T \otimes_{F} R \rightarrow T \otimes_{K} U
$$

where (again) the $\theta$-structure is extended trivially to $T \otimes_{K} U$.

(Actually $U$ is isomorphic to the ring of constants of $T \otimes_{F} R$.)

Proof. $R$ is obtained as a quotient of $F\left[X, X^{-1}\right]$ with $\theta$-structure given by $\theta(X)=$ $A X$ (for short we write $F\left[X, X^{-1}\right]$ instead of $F\left[X_{i j}, \operatorname{det}(X)^{-1}\right]$ ) by a maximal $\theta$ ideal $P \unlhd F\left[X, X^{-1}\right]$. Let $L:=K\left[Z, Z^{-1}\right]=K\left[\mathrm{GL}_{n}\right]$. We then define a $T$-linear homomorphism

$$
\gamma_{T}: T \otimes_{F} F\left[X, X^{-1}\right] \rightarrow T \otimes_{K} K\left[Z, Z^{-1}\right]
$$

by $X_{i j} \mapsto \sum_{k=1}^{n} Y_{i k} \otimes Z_{k j}$ (or $X \mapsto Y \otimes Z$ for short). $\gamma_{T}$ is indeed an isomorphism and, if we extend the $\theta$-structure trivially to $T \otimes_{K} K\left[Z, Z^{-1}\right], \gamma_{T}$ is a $\theta$-isomorphism.

By the previous lemma, the $\theta$-ideal $\gamma_{T}(T \otimes P)$ is equal to $T \otimes I$ for an ideal $I \unlhd K\left[Z, Z^{-1}\right]$. So for $U:=K\left[Z, Z^{-1}\right] / I, \gamma_{T}$ induces a $\theta$-isomorphism

$$
\gamma_{T}: T \otimes_{F} R \rightarrow T \otimes_{K} U
$$

Proposition 10.9. Let $R$ be a $P P V$-ring over $F$. Then the group functor $\operatorname{Aut}^{\theta}(R / F)$ is represented by the finitely generated $K$-algebra $U=C_{R \otimes_{F} R}$, i.e. Aut $^{\theta}(R / F)$ is an affine group scheme of finite type over $K$, which we call the Galois group scheme $\operatorname{Gal}(R / F)$ of $R$ over $F$ or also the Galois group scheme $\operatorname{Gal}(E / F)$ of $E:=\operatorname{Quot}(R)$ over $F$.

Proof. First we show that for every $K$-algebra $L$, any $F_{L}$-linear $\theta$-homomorphism $f: R_{L} \rightarrow R_{L}$ is an isomorphism: The kernel of such a homomorphism $f$ is a $\theta$ ideal of $R_{L}$, and so by Lemma 10.7 it is generated by constants, i.e. elements in $1 \otimes L$. But $f$ is $L$-linear, and so $\operatorname{Ker}(f)=\{0\}$. If $X \in \mathrm{GL}_{n}(R)$ is a fundamental matrix, then $f(X) \in \mathrm{GL}_{n}\left(R_{L}\right)$ is also a fundamental matrix, and so there is a matrix $D \in \mathrm{GL}_{n}\left(C_{R_{L}}\right)=\mathrm{GL}_{n}(L)$ such that $X=f(X) D=f(X D)$. Hence $X_{i j}, \operatorname{det}(X)^{-1} \in \operatorname{Im}(f)(i, j=1, \ldots, n)$, and since $R$ is generated over $F$ by the $X_{i j}$ and by $\operatorname{det}(X)^{-1}$, the homomorphism $f$ is also surjective. 
Using the isomorphism $\gamma:=\gamma_{R}$ of Proposition 10.8, for a $K$-algebra $L$, we obtain a chain of isomorphisms:

$$
\begin{aligned}
\operatorname{Aut}^{\theta}\left(R_{L} / F_{L}\right) & =\operatorname{Hom}_{F_{L}}^{\theta}\left(R_{L}, R_{L}\right)=\operatorname{Hom}_{F_{L}}^{\theta}\left(F_{L} \otimes_{F} R, R_{L}\right) \\
& \cong \operatorname{Hom}_{R}^{\theta}\left(R \otimes_{F} R, R_{L}\right) \\
& \cong \operatorname{Hom}_{R}^{\theta}\left(R \otimes_{K} U, R_{L}\right) \\
& \cong \operatorname{Hom}_{K}^{\theta}\left(U, R_{L}\right) \\
& \cong \operatorname{Hom}_{K}(U, L)
\end{aligned}
$$

So $U$ is representing the functor $\underline{\operatorname{Aut}}^{\theta}(R / F)$.

Remark 10.10. A careful look at the isomorphisms in the previous proof shows that the universal object $\operatorname{id}_{U} \in \operatorname{Hom}_{K}(U, U)$ corresponds to the $\theta$-automorphism $\rho \otimes \operatorname{id}_{U}: R \otimes_{K} U \rightarrow R \otimes_{K} U$, where $\rho=\gamma_{R} \circ\left(1 \otimes \operatorname{id}_{R}\right): R \rightarrow R \otimes_{F} R \rightarrow R \otimes_{K} U$. Furthermore, we obtain that the action of $g \in \underline{\operatorname{Aut}^{\theta}}(R / F)(L)=\operatorname{Hom}_{K}(U, L)$ on $r \in R$ is given by

$$
g \cdot r=\left(\operatorname{id}_{R} \otimes g\right)\left(\gamma_{R}(1 \otimes r)\right) \in R \otimes_{K} L .
$$

Corollary 10.11. Let $R$ be a PPV-ring over $F$ and $\mathcal{G}:=\underline{\mathrm{Gal}}(R / F)$ be the Galois group scheme of $R$. Then $\operatorname{Spec}(R)$ is a $\mathcal{G}_{F}$-torsor.

Proof. The isomorphism $\gamma=\gamma_{R}$ of Proposition 10.8 determines an isomorphism of schemes

$$
\operatorname{Spec}(\gamma): \operatorname{Spec}(R) \times{ }_{F} \mathcal{G}_{F}=\operatorname{Spec}(R) \times_{K} \mathcal{G} \rightarrow \operatorname{Spec}(R) \times_{F} \operatorname{Spec}(R)
$$

By the previous remark and $R$-linearity of $\gamma$, the composition of $\operatorname{Spec}(\gamma)$ with the second projection is the morphism which describes the action of $\mathcal{G}_{F}$ on $\operatorname{Spec}(R)$, and the composition of $\operatorname{Spec}(\gamma)$ with the first projection is equal to the first projection $\operatorname{Spec}(R) \times{ }_{F} \mathcal{G}_{F} \rightarrow \operatorname{Spec}(R)$. In other words, $\operatorname{Spec}(R)$ is a $\mathcal{G}_{F}$-torsor.

The next proposition shows that being a torsor indicates a $\theta$-simple $\theta$-ring to be a PPV-ring.

Proposition 10.12. Let $R / F$ be a $\theta$-simple $\theta$-ring with constants $C_{R}=K$. Further, let $\mathcal{G} \leq \mathrm{GL}_{n, K}$ be an affine group scheme over $K$ and assume that $\operatorname{Spec}(R)$ is a $\mathcal{G}_{F}$-torsor such that the corresponding isomorphism $\gamma: R \otimes_{F} R \rightarrow R \otimes_{K} K[\mathcal{G}]$ is a $\theta$-isomorphism. Then $R$ is a PPV-ring over $F$.

Proof. Since $\operatorname{Spec}(R)$ is a $\mathcal{G}_{F}$-torsor, the fibration $\operatorname{Spec}(R) \times \mathcal{G}_{F} \mathrm{GL}_{n, F}$ is a $\mathrm{GL}_{n, F^{-}}$ torsor. (The scheme $\operatorname{Spec}(R) \times \mathcal{G}_{F} \mathrm{GL}_{n, F}$ is obtained as the quotient of the direct product by the action of $\mathcal{G}_{F}$ given by $(x, h) . g:=\left(x g, g^{-1} h\right)$, and is a right $\mathrm{GL}_{n, F^{-}}$ scheme by the action on the second factor.) By the Hilbert 90 theorem, every $\mathrm{GL}_{n, F}$-torsor is trivial, i.e. we have a $\mathrm{GL}_{n, F}$-equivariant isomorphism $\operatorname{Spec}(R) \times \mathcal{G}_{F}$ $\mathrm{GL}_{n, F} \rightarrow \mathrm{GL}_{n, F}$. Then the closed embedding $\operatorname{Spec}(R) \hookrightarrow \operatorname{Spec}(R) \times \mathcal{G}_{F} \mathrm{GL}_{n, F} \rightarrow$ $\mathrm{GL}_{n, F}$ induces an epimorphism $F\left[X, X^{-1}\right] \rightarrow R$ which is $\mathcal{G}_{F}$-equivariant. Let the image of $X$ be denoted by $Y$. We then obtain that the action of $\mathcal{G}$ on $Y$ is given by $Y \mapsto Y \cdot g$ for any $L$-valued point $g \in \mathcal{G}(L) \subset \mathrm{GL}_{n}(L)$. Since by assumption for every $K$-algebra $L$ the action of $\mathcal{G}(L)$ commutes with $\theta$, the matrix $\theta(Y) Y^{-1}$ is $\mathcal{G}$-invariant. So $\theta(Y) Y^{-1}=: A \in \mathrm{GL}_{n}(\tilde{\Omega})$, and $Y$ is a fundamental solution matrix for the equation $\theta(\boldsymbol{y})=A \boldsymbol{y}$. Hence $R$ is a PPV-ring. 
Remark 10.13. As indicated at the beginning of this section, the Tannakian Galois group scheme $G_{(M, \nabla)}$ of a module with iterative connection and the Galois group scheme of a PPV-extension for $(M, \nabla)$ are isomorphic. We now sketch this isomorphism.

So let $K$ be a perfect field, and let $S$ be a regular integral domain which is the localisation of a finitely generated $K$-algebra, and such that there is a maximal ideal $\mathfrak{m} \unlhd S$ with $S / \mathfrak{m} \cong K$. Furthermore, let $(M, \nabla) \in \operatorname{ICon}(S / K)$, and let $F:=\operatorname{Quot}(S)$ denote the quotient field of $S$ and $\theta:=\mathrm{d}_{F}: F \rightarrow \hat{\Omega}_{F / K}$ the universal derivation of $F$. Since $M$ is a locally free module (cf. Corollary 4.5), by Hart77, Ch. II, Lemma 8.9 , there exists a basis $\boldsymbol{b}:=\left(b_{1}, \ldots, b_{n}\right)$ of the $F$-vector space $F \otimes_{S} M$ with $b_{i} \in M(i=1, \ldots, n)$, and such that the residue classes in $M / \mathfrak{m} M$ form a $K$-basis of $M / \mathfrak{m} M$. We assume that there exists a PPV-ring $R$ for the corresponding iterable differential equation $\nabla(\boldsymbol{b} \boldsymbol{x})=\boldsymbol{b} \boldsymbol{x}$ with fundamental solution matrix $Y \in \mathrm{GL}_{n}(R)$.

For obtaining the correspondence, we fix an isomorphism of $R$-modules $\varphi: R \otimes_{S}$ $M \rightarrow R \otimes_{S} M$ given by $\varphi(\boldsymbol{b})=\boldsymbol{b} Y$. The correspondence is then given as follows:

For any $K$-algebra $L$ (with trivial $\theta$-structure), an element $\sigma \in G_{(M, \nabla)}(L)$ is determined by $\sigma_{M} \in \mathrm{GL}\left(L \otimes_{K} \boldsymbol{\omega}_{K}(M)\right)$, which can be identified with a matrix $D_{\sigma} \in \mathrm{GL}_{n}(L)$ by $\sigma_{M}(\boldsymbol{b})=\boldsymbol{b} D_{\sigma}$. So $\sigma_{M}$ induces an $\left(R \otimes_{K} L\right)$-linear automorphism $\tilde{\sigma}_{M}$ of $\left(R \otimes_{K} L\right) \otimes_{S} M$ by $\boldsymbol{b} \mapsto \boldsymbol{b} D_{\sigma}$, and we obtain a $\theta$-isomorphism $\hat{\sigma}:=\varphi \circ \tilde{\sigma}_{M} \circ \varphi^{-1}$ of $\left(R \otimes_{K} L\right) \otimes_{S} M$ mapping the constant basis $\boldsymbol{b} Y$ to the constant basis $\boldsymbol{b} Y D_{\sigma}$. One shows that this induces a $\theta$-isomorphism of $R \otimes_{K} L$ over $F \otimes_{K} L$ given by $Y \mapsto Y D_{\sigma}$, i.e. an element of $\underline{\operatorname{Gal}}(R / F)(L)$.

On the other hand, every $\theta$-isomorphism of $R \otimes_{K} L$ over $F \otimes_{K} L$ is given by $Y \mapsto Y D$ for some $D \in \mathrm{GL}_{n}(L)$, and by reversing the steps above, one obtains an element $\sigma_{M} \in \mathrm{GL}\left(L \otimes_{K} \boldsymbol{\omega}_{K}(M)\right)$ and one shows that indeed $\sigma_{M}$ defines an element $\sigma \in G_{(M, \nabla)}(L)$.

\section{Galois CORRESPONDENCE}

In this section, we prove a Galois correspondence between all intermediate $\theta$ fields of a PPV-extension $E / F$ and all closed subgroup schemes of the Galois group scheme $\operatorname{Gal}(E / F)$. This includes $\theta$-fields over which $E$ is inseparable and nonreduced subgroup schemes, and hence is an improvement of the correspondence given by Matzat and van der Put (cf. MvdP03, Thm. 3.5), which only considers reduced subgroup schemes and intermediate fields over which $E$ is separable. (However, this separability condition is missing in their statement, but has been added for example in [Ama07, Thm. 2.5, and in [Hei07, Thm. 6.5.2.)

Remark 11.1. One should also mention the work of M. Takeuchi (cf. Tak89) on a Picard-Vessiot theory of so-called C-ferential fields (a huge class of fields with extra structure to which the iterative differential fields and the $\theta$-fields defined below belong). But Takeuchi used a definition of a PV-extension that differs from ours and from the "usual" one. The main difference is that instead of requiring the existence of a fundamental solution matrix, he imposed a condition which is equivalent to an isomorphism $R \otimes_{F} R \cong R \otimes_{K} C_{R \otimes_{F} R}$. (Here $F$ denotes a Cferential field, $R$ a PV-ring over $F, K=C_{F}=C_{R}$ the field of constants of $F$ and $R$, and $C_{R \otimes_{F} R}$ the constants of $R \otimes_{F} R$; cf. Tak89, Def. 2.3). Showing that this isomorphism also exists by our definition was the statement of Proposition 10.8. In fact, Proposition 10.8 and Proposition 10.12 imply that both definitions coincide 
in the case of $\theta$-fields. Our Galois correspondence is quite the same as the one given by Takeuchi (cf. Tak89], Thm 2.10), but we give maps in both directions (Takeuchi only constructed the subgroup scheme corresponding to an intermediate field) and also include the correspondence of the separability condition and the reducedness condition (separability and reducedness are not mentioned at all in Takeuchi's work).

In order to provide the Galois correspondence for PPV-extensions, we need a functorial definition of invariants. Let $S$ be a $K$-algebra and $\mathcal{H} / K$ be a subgroup functor of the functor $\operatorname{Aut}(S / K)$; i.e. for every $K$-algebra $L$, the set $\mathcal{H}(L)$ is a group acting on $S_{L}$ and this action is functorial in $L$. An element $s \in S$ is then called invariant if for all $L$, the element $s \otimes 1 \in S_{L}$ is invariant under $\mathcal{H}(L)$. The ring of invariants is denoted by $S^{\mathcal{H}}$. (In Jan03, I.2.10, the invariant elements are called "fixed points".) Let $E=\operatorname{Quot}(S)$ be the localisation of $S$ by all nonzero divisors. We call an element $e=\frac{r}{s} \in E$ invariant under $\mathcal{H}$ if for all $K$-algebras $L$ and all $h \in \mathcal{H}(L)$,

$$
h \cdot(r \otimes 1) \cdot(s \otimes 1)=(r \otimes 1) \cdot h \cdot(s \otimes 1) \in S \otimes_{K} L .
$$

The ring of invariants of $E$ is denoted by $E^{\mathcal{H}}$. One can easily verify that this definition of an invariant element $e \in E$ is independent of the chosen representation $\frac{r}{s}$.

Remark 11.2. One has to take care that in general the group functor $\operatorname{Aut}(S / K)$ is not a subgroup functor of $\underline{\operatorname{Aut}}(E / K)$, because not every automorphism $S \otimes_{K} L \rightarrow$ $S \otimes_{K} L$ can be extended to an automorphism $E \otimes_{K} L \rightarrow E \otimes_{K} L$. Hence a subgroup functor $\mathcal{H}$ of $\underline{\operatorname{Aut}}(S / K)$ does not have to be a subgroup functor of $\underline{\operatorname{Aut}}(E / K)$. That is why we use this more complicated definition of the invariants $\overline{E^{\mathcal{H}}}$.

In the following, let $R$ be a PPV-ring over $F, E=\operatorname{Quot}(R)$ its quotient field and $\mathcal{G}=\underline{\operatorname{Gal}}(R / F)$ the Galois group scheme of $R$ over $F$.

Lemma 11.3. Let $\mathcal{H} \leq \mathcal{G}$ be a closed subgroup scheme and let $\pi_{\mathcal{H}}^{\mathcal{G}}: K[\mathcal{G}] \rightarrow K[\mathcal{H}]$ denote the epimorphism corresponding to the inclusion $\mathcal{H} \hookrightarrow \mathcal{G}$. Then an element $\frac{r}{s} \in E$ is invariant under the action of $\mathcal{H}$ if and only if $r \otimes s-s \otimes r$ is in the kernel of the map

$$
\left(\operatorname{id}_{R} \otimes \pi_{\mathcal{H}}^{\mathcal{G}}\right) \circ \gamma: R \otimes_{F} R \rightarrow R \otimes_{K} K[\mathcal{H}]
$$

Proof. An element $\frac{r}{s} \in E$ is invariant under the action of $\mathcal{H}$ if and only if it is invariant under the universal element in $\mathcal{H}$, namely $\pi_{\mathcal{H}}^{\mathcal{G}} \in \mathcal{G}(K[\mathcal{H}])$. By Remark 10.10 and $R$-linearity of $\gamma$, we have

$$
\left(\operatorname{id}_{R} \otimes \pi_{\mathcal{H}}^{\mathcal{G}}\right)(\gamma(r \otimes s))=(r \otimes 1) \cdot \pi_{\mathcal{H}}^{\mathcal{G}} \cdot(s \otimes 1) \in R \otimes_{K} K[\mathcal{H}]
$$

Hence $r \otimes s-s \otimes r$ is in the considered kernel if and only if $\frac{r}{s}$ is invariant under $\mathcal{H}$.

Theorem 11.4. For every closed subgroup scheme $\mathcal{H} \leq \mathcal{G}$, the ring $E^{\mathcal{H}}$ is a $\theta$-field. Furthermore, we have $E^{\mathcal{H}}=F$ if and only if $\mathcal{H}=\mathcal{G}$. 
Proof. By the previous lemma, it is obvious that $E^{\mathcal{H}}$ is a field. Next let $\frac{r}{s} \in E^{\mathcal{H}}$. Then for all $k \in \mathbb{N}$, we have

$$
\begin{aligned}
& \theta^{(k)}(r \otimes s-s \otimes r) \cdot\left(s^{k} \otimes s^{k}\right) \\
= & \sum_{i_{1}+i_{2}+i_{3}=k} \theta^{\left(i_{1}\right)}\left(\frac{r}{s}\right) s^{k} \theta^{\left(i_{2}\right)}(s) \otimes \theta^{\left(i_{3}\right)}(s) s^{k}-\theta^{\left(i_{2}\right)}(s) s^{k} \otimes \theta^{\left(i_{1}\right)}\left(\frac{r}{s}\right) s^{k} \theta^{\left(i_{3}\right)}(s) \\
= & \sum_{i_{1}+i_{2}+i_{3}=k}\left(\theta^{\left(i_{2}\right)}(s) \otimes \theta^{\left(i_{3}\right)}(s)\right)\left(\theta^{\left(i_{1}\right)}\left(\frac{r}{s}\right) s^{k} \otimes s^{k}-s^{k} \otimes \theta^{\left(i_{1}\right)}\left(\frac{r}{s}\right) s^{k}\right) \\
= & \sum_{i+j=k} \theta^{(i)}(s \otimes s)\left(\theta^{(j)}\left(\frac{r}{s}\right) s^{k} \otimes s^{k}-s^{k} \otimes \theta^{(j)}\left(\frac{r}{s}\right) s^{k}\right) .
\end{aligned}
$$

The left hand side lies in $\operatorname{Ker}\left(\left(\operatorname{id}_{R} \otimes \pi_{\mathcal{H}}^{\mathcal{G}}\right) \circ \gamma\right) \otimes \tilde{\Omega}_{k}$, since the kernel is a $\theta$-ideal. So by induction, we obtain

$$
(s \otimes s)\left(\theta^{(k)}\left(\frac{r}{s}\right) s^{k} \otimes s^{k}-s^{k} \otimes \theta^{(k)}\left(\frac{r}{s}\right) s^{k}\right) \in \operatorname{Ker}\left(\left(\operatorname{id}_{R} \otimes \pi_{\mathcal{H}}^{\mathcal{G}}\right) \circ \gamma\right) \otimes \tilde{\Omega}_{k}
$$

and hence $\theta^{(k)}\left(\frac{r}{s}\right) \in E^{\mathcal{H}} \otimes \tilde{\Omega}_{k}$.

For the second statement: If $\mathcal{H}=\mathcal{G}$, then $\pi_{\mathcal{H}}^{\mathcal{G}}=\mathrm{id}_{K[\mathcal{G}]}$ and the considered kernel is trivial. Hence $r \otimes s=s \otimes r \in R \otimes_{F} R$ for all $\frac{r}{s} \in E^{\mathcal{G}}$. So $r=c \cdot s$ for an appropriate element $c \in F$, i.e. $\frac{r}{s}=c \in F$.

Assume $\mathcal{H} \lesseqgtr \mathcal{G}$. Since $\mathcal{X}=\operatorname{Spec}(R)$ is a $\mathcal{G}_{F}$-torsor, the quotient scheme $\mathcal{X} / \mathcal{G}_{F}$ is equal to $\operatorname{Spec}(F)$. In particular it is a scheme, and since $\mathcal{G}_{F}$ and $\mathcal{H}_{F}$ are affine, $\mathcal{G}_{F} / \mathcal{H}_{F}$ also is a scheme. So by [Jan03], I.5.16(1), $\mathcal{X} / \mathcal{H}_{F} \cong \mathcal{X} \times \mathcal{G}_{F}\left(\mathcal{G}_{F} / \mathcal{H}_{F}\right)$ is a scheme, too. Let $\overline{\mathcal{U}} \subset \mathcal{X} / \mathcal{H}_{F}$ be an arbitrary affine open subset and $\mathcal{U}=$ $\operatorname{pr}^{-1}(\overline{\mathcal{U}}) \subset \mathcal{X}$ be its inverse image, where pr: $\mathcal{X} \rightarrow \mathcal{X} / \mathcal{H}_{F}$ denotes the canonical projection. Then we get a monomorphism $\operatorname{pr}_{*}: \mathcal{O}_{\mathcal{X} / \mathcal{H}_{F}}(\overline{\mathcal{U}}) \rightarrow \mathcal{O}_{\mathcal{X}}(\mathcal{U})$, whose image is $\mathcal{O}_{\mathcal{X}}(\mathcal{U})^{\mathcal{H}}$. If $E^{\mathcal{H}}=F$, then also $\mathcal{O}_{\mathcal{X}}(\mathcal{U})^{\mathcal{H}}=F$. So for every open affine subset $\overline{\mathcal{U}} \subset \mathcal{X} / \mathcal{H}_{F}$ we would have $\mathcal{O}_{\mathcal{X} / \mathcal{H}_{F}}(\overline{\mathcal{U}})=F$, i.e. $\overline{\mathcal{U}} \cong \operatorname{Spec}(F)$ is a single point. Hence $\mathcal{X} / \mathcal{H}_{F}=\operatorname{Spec}(F)$, which contradicts the assumption $\mathcal{H} \lesseqgtr \mathcal{G}$.

Theorem 11.5 (Galois correspondence).

i) There is an anti-isomorphism of the lattices

$$
\mathfrak{H}:=\{\mathcal{H} \mid \mathcal{H} \leq \mathcal{G} \text { closed subgroup schemes of } \mathcal{G}\}
$$

and

$$
\mathfrak{M}:=\{M \mid F \leq M \leq E \text { intermediate } \theta \text {-fields }\}
$$

given by $\Psi: \mathfrak{H} \rightarrow \mathfrak{M}, \mathcal{H} \mapsto E^{\mathcal{H}}$ and $\Phi: \mathfrak{M} \rightarrow \mathfrak{H}, M \mapsto \underline{\operatorname{Gal}}(R M / M)$.

ii) If $\mathcal{H} \leq \mathcal{G}$ is normal, then $E^{\mathcal{H}}=\operatorname{Quot}\left(R^{\mathcal{H}}\right)$ and $R^{\mathcal{H}}$ is a PPV-ring over $F$ with Galois group scheme $\operatorname{Gal}\left(R^{\mathcal{H}} / F\right) \cong \mathcal{G} / \mathcal{H}$.

iii) If $M \in \mathfrak{M}$ is stable under the action of $\mathcal{G}$, then $\mathcal{H}:=\Phi(M)$ is a normal subgroup scheme of $\mathcal{G}, M$ is a PPV-extension of $F$ and $\underline{\mathrm{Gal}}(R \cap M / F) \cong$ $\mathcal{G} / \mathcal{H}$.

iv) For $\mathcal{H} \in \mathfrak{H}$, the extension $E / E^{\mathcal{H}}$ is separable if and only if $\mathcal{H}$ is reduced.

Proof. i) Let $M \in \mathfrak{M}$ be an intermediate $\theta$-field. Then the composite $R M \subseteq E$ of $R$ and $M$ is a PPV-ring over $M$. Furthermore, the canonical $\theta$-epimorphism $R M \otimes_{F} R \rightarrow R M \otimes_{M} R M$ gives rise to a $\theta$-epimorphism

$$
R M \otimes_{K} K[\mathcal{G}] \stackrel{\gamma_{R M}^{-1}}{\longrightarrow} R M \otimes_{F} R \rightarrow R M \otimes_{M} R M .
$$


By Lemma 10.7 the kernel of this epimorphism is given by $R M \otimes_{K} I$ for an ideal $I \unlhd K[\mathcal{G}]$. Let $\mathcal{H}$ denote the closed subscheme of $\mathcal{G}$ defined by $I$. Then $\gamma_{R M}$ induces an isomorphism

$$
R M \otimes_{M} R M \stackrel{\cong}{\rightarrow} R M \otimes_{K} K[\mathcal{H}] .
$$

By construction, this isomorphism is the isomorphism $\gamma$ for the base field $M$. Hence the subscheme $\mathcal{H}$ equals the Galois group scheme $\operatorname{Gal}(R M / M)$. So $\operatorname{Gal}(R M / M)$ is indeed a closed subgroup scheme of $\mathcal{G}$.

From Theorem 11.4 applied to the extension $E / M$, we see that $E \underline{\mathrm{Gal}}(R M / M)=$ $M$, so $\Psi \circ \Phi=\mathrm{id}_{\mathfrak{M}}$. On the other hand, for given $\mathcal{H} \in \mathfrak{H}$ and $M:=E^{\mathcal{H}}$, we obtain a $\theta$-epimorphism $R M \otimes_{M} R M \rightarrow R M \otimes_{K} K[\mathcal{H}]$ induced from $\gamma_{R M}$. This gives $\mathcal{H}$ as a closed subgroup scheme of $\operatorname{Gal}(R M / M)$. But $(\operatorname{Quot}(R M))^{\mathcal{H}}=E^{\mathcal{H}}=M$, and so by Theorem 11.4, we have $\mathcal{H}=\underline{\operatorname{Gal}}(R M / M)$. Hence $\Phi \circ \Psi=\mathrm{id}_{\mathfrak{H}}$.

ii) Let $\mathcal{H} \leq \mathcal{G}$ be normal. The isomorphism $\gamma$ is $\mathcal{H}$-equivariant (by the action of $\mathcal{H}$ on the right factor), and hence we get a $\theta$-isomorphism

$$
R \otimes_{F} R^{\mathcal{H}} \cong R \otimes_{K} K[\mathcal{G}]^{\mathcal{H}} .
$$

Since $\mathcal{H}$ is normal, $\mathcal{G} / \mathcal{H}$ is an affine group scheme with $K[\mathcal{G} / \mathcal{H}] \cong K[\mathcal{G}]^{\mathcal{H}}$ (cf. DG70], III, $\S 3$, Thm. 5.6 and 5.8). Again by taking invariants (this time $\mathcal{H}$ acting on the first factor) the isomorphism above restricts to an isomorphism

$$
R^{\mathcal{H}} \otimes_{F} R^{\mathcal{H}} \cong R^{\mathcal{H}} \otimes_{K} K[\mathcal{G} / \mathcal{H}] .
$$

$R^{\mathcal{H}}$ is $\theta$-simple, because for every $\theta$-ideal $P \unlhd R^{\mathcal{H}}$, the ideal $P \cdot R \unlhd R$ is a $\theta$-ideal, hence equals $(0)$ or $R$, and so $P=(P \cdot R)^{\overline{\mathcal{H}}}$ is $(0)$ or $R^{\mathcal{H}}$. Since $F \leq R^{\mathcal{H}} \leq R$, we also have $C_{R^{\mathcal{H}}}=K$. So by Proposition 10.12, $R^{\mathcal{H}}$ is a PPV-ring over $F$ with Galois group scheme $\mathcal{G} / \mathcal{H}$. It remains to show that $E^{\mathcal{H}}=\operatorname{Quot}\left(R^{\mathcal{H}}\right)$ :

Let $\tilde{F}:=\operatorname{Quot}\left(R^{\mathcal{H}}\right)$ and $\tilde{\mathcal{G}}:=\underline{\operatorname{Gal}}(E / \tilde{F})$. Then $\mathcal{H}$ is a normal subgroup of $\tilde{\mathcal{G}}$ and by the same argument as before, $(R \cdot \tilde{F})^{\mathcal{H}}$ is a $(\tilde{\mathcal{G}} / \mathcal{H})_{\tilde{F}}$-torsor. But $(R \cdot \tilde{F})^{\mathcal{H}}=$ $R^{\mathcal{H}} \cdot \tilde{F}=\tilde{F}$, so $\tilde{\mathcal{G}}=\mathcal{H}$, and hence $E^{\mathcal{H}}=E^{\tilde{\mathcal{G}}}=\tilde{F}=\operatorname{Quot}\left(R^{\mathcal{H}}\right)$.

iii) It suffices to show that $\mathcal{H}$ is normal in $\mathcal{G}$. The rest then follows from ii). Let $L$ be a $K$-algebra and let $h \in \mathcal{H}(L)$ and $g \in \mathcal{G}(L)$. Then for all $r \in R \cap M$, we have

$$
g h g^{-1} \cdot(r \otimes 1)=g h \cdot\left(g^{-1} \cdot(r \otimes 1)\right)=g \cdot\left(g^{-1} \cdot(r \otimes 1)\right)=(r \otimes 1),
$$

since $g^{-1} .(r \otimes 1) \in(R \cap M) \otimes_{K} L$ by $\mathcal{G}$-stability of $M$. So $g h g^{-1} \in \mathcal{H}(L)$, and therefore $\mathcal{H}$ is normal in $\mathcal{G}$.

iv) Without loss of generality let $\mathcal{H}=\mathcal{G}$. If $\mathcal{G}$ is reduced, then $F=E^{\mathcal{G}}=E^{\mathcal{G}(\bar{K})}$ and hence $E \otimes_{K} \bar{K} / F \otimes_{K} \bar{K}$ is separable, and so $E / F$ is separable. On the other hand, if $\mathcal{G}$ is not reduced, then $R \otimes_{F} R \cong R \otimes_{K} K[\mathcal{G}]$ is not reduced. Hence, $E \otimes_{F} E$ is not reduced. But this is just one criterion for $E / F$ being inseparable (cf. [Mats89, beginning of Sect. 26).

Corollary 11.6. Let $E / F$ be a PPV-extension with Galois group scheme $\mathcal{G}$. Then $E / F$ is a purely inseparable extension if and only if $\mathcal{G}$ is an infinitesimal group scheme.

Proof. Let $\mathcal{G}$ be infinitesimal, and let $e v: K[\mathcal{G}] \rightarrow K$ denote the evaluation map corresponding to the neutral element of the group. Then for any $\frac{r}{s} \in E$, we have $(\operatorname{id} \otimes e v)(\gamma(r \otimes s-s \otimes r))=0$. Since $\mathcal{G}$ is infinitesimal, the kernel of $e v$ is the 
nilradical, and hence there is some $k \in \mathbb{N}$ such that $(r \otimes s-s \otimes r)^{p^{k}}=0$, where $p=\operatorname{char}(F)$. Therefore, $r^{p^{k}} \otimes s^{p^{k}}=s^{p^{k}} \otimes r^{p^{k}} \in E \otimes_{F} E$, which means that $\frac{r^{p^{k}}}{s^{p^{k}}} \in F$. So $E / F$ is purely inseparable. On the other hand, if $E / F$ is purely inseparable, then $\mathcal{G}(\bar{K})=\operatorname{Aut}^{\theta}\left(E \otimes_{K} \bar{K} / F \otimes_{K} \bar{K}\right)$ is the trivial group, since $E \otimes_{K} \bar{K} / F \otimes_{K} \bar{K}$ is also a purely inseparable extension. Hence $\mathcal{G}$ is infinitesimal.

Corollary 11.7. Let $p:=\operatorname{char}(F)>0$. If $\operatorname{Ker}\left(\theta_{F}^{(1)}\right)=F^{p}$, then all PPV-extensions $E / F$ are separable, and the corresponding Galois group schemes are reduced.

Proof. By Theorem 11.5v), the separability of a PPV-extension $E / F$ is equivalent to the reducedness of $\mathrm{Gal}(E / F)$. Assume there exists an inseparable PPV-extension $E / F$. Then there is an inseparable element $e \in E$ with minimal polynomial $f(X)$ in $F\left[X^{p}\right]$. Since $\theta_{E}^{(1)}(f(e))=0$, all coefficients of $f$ are in $\operatorname{Ker}\left(\theta_{F}^{(1)}\right)=F^{p}$. But then $f(X)=g(X)^{p}$ for some $g(X) \in F[X]$, which contradicts the irreducibility of $f$.

Example 11.8. We consider some examples for subfields of $(K((t)), \theta)$, where $\theta:=\phi_{t} \in \mathrm{ID}_{K}(K((t)))$ is the iterative derivation with respect to $t$ (cf. Example 3.3) and $K$ denotes a field of characteristic $p>0$. For simplicity we assume that $K$ is algebraically closed.

(i) Let $F=K(t) \subseteq K((t))$. Then the IDE given by

$$
\theta^{\left(p^{l}\right)}(y)=a_{l} t^{-p^{l}} y \quad\left(a_{l} \in K\right)
$$

has a PPV-ring $R=F\left[s, s^{-1}\right]$ and PPV-field $E=F(s)$, where $s$ is a solution of the IDE. This implies that $\operatorname{Gal}(E / F)$ is a subgroup of $\mathbb{G}_{m}$. If the $a_{l}$ are chosen appropriately, then we have $\underline{\operatorname{Gal}}(E / F)=\mathbb{G}_{m}$ (cf. [Mat01, Thm. 3.13, resp. MvdP03, Section 4) and $s$ is transcendental over $F$. Furthermore, the isomorphism $\gamma: R \otimes_{F} R \rightarrow R \otimes_{K} K\left[\mathbb{G}_{m}\right]$ is given by $1 \otimes s \mapsto s \otimes x\left(K\left[\mathbb{G}_{m}\right]=: K\left[x, x^{-1}\right]\right)$.

All closed subgroup schemes of $\mathbb{G}_{m}$ are given by the ideals $\left(x^{k}-1\right) \unlhd$ $K\left[x, x^{-1}\right]$ for $k \in \mathbb{N}$ (the so-called subgroups $\mu_{k}$ of $k$-th roots of unity), and the corresponding intermediate $\theta$-fields are $E^{\mu_{k}}=\operatorname{Quot}\left(R^{\mu_{k}}\right)=F\left(s^{k}\right)$. Hence, there are also intermediate $\theta$-fields over which $E$ is inseparable, namely for all $k>0$ that are divisible by $p$.

(ii) Let $F \subseteq K((t))$ be the subfield generated over $K$ by $t, s_{1}:=\prod_{l=0}^{\infty}\left(1+t^{a_{l} p^{l}}\right)$ and $s_{2}:=\prod_{l=0}^{\infty}\left(1+t^{b_{l} p^{l}}\right)$, where $a_{l}, b_{l} \in\{0,1, \ldots, p-1\}$ are chosen such that $t, s_{1}$ and $s_{2}$ are algebraically independent. Consider the IDE

$$
\theta^{\left(p^{l}\right)}\left(\begin{array}{l}
y_{1} \\
y_{2}
\end{array}\right)=\left(\begin{array}{cc}
a_{l+1}\left(1+t^{a_{l+1} p^{l+1}}\right)^{-1} & 0 \\
0 & b_{l+1}\left(1+t^{b_{l+1} p^{l+1}}\right)^{-1}
\end{array}\right)\left(\begin{array}{l}
y_{1} \\
y_{2}
\end{array}\right) \quad(l \in \mathbb{N}) .
$$

A solution of this IDE is given by $\left(\begin{array}{l}r_{1} \\ r_{2}\end{array}\right) \in K((t))^{2}$ with $r_{1}^{p}=\left(1+t^{a_{0}}\right)^{-1} \cdot s_{1}$ and $r_{2}^{p}=\left(1+t^{b_{0}}\right)^{-1} \cdot s_{2}$. Hence the corresponding PPV-ring is $R=F\left[r_{1}, r_{2}\right]$ and the Galois group scheme - a priori a subgroup of $\mathbb{G}_{m} \times \mathbb{G}_{m}$ - is equal to $\mu_{p} \times \mu_{p}$. The action of the Galois group scheme on $R$ is given by the homomorphism $\rho: R \rightarrow R \otimes_{K} K\left[\mu_{p} \times \mu_{p}\right] \cong R \otimes_{K} K\left[x_{1}, x_{2}\right] /\left(x_{1}^{p}-1, x_{2}^{p}-1\right)$, which maps $r_{i}$ to $r_{i} \otimes x_{i}(i=1,2)$. Since the nontrivial subgroups of $\mu_{p} \times \mu_{p}$ are given by the ideals $\left(x_{1}^{k} x_{2}-1\right) \unlhd K\left[x_{1}, x_{2}\right] /\left(x_{1}^{p}-1, x_{2}^{p}-1\right)$ $(k \in\{0,1, \ldots, p-1\})$ and $\left(x_{1}-1\right) \unlhd K\left[x_{1}, x_{2}\right] /\left(x_{1}^{p}-1, x_{2}^{p}-1\right)$, there are 
exactly $p+1$ intermediate $\theta$-fields unequal to $E$ and $F$, namely $F\left(r_{1}^{k} r_{2}\right)$, resp. $F\left(r_{1}\right)$.

So in this case, although $E / F$ has infinitely many intermediate fields, there are only finitely many intermediate $\theta$-fields.

(iii) Let $F \subseteq K((t))$ be the subfield generated over $K$ by $t, s_{1}:=\sum_{l=0}^{\infty} a_{l} t^{p^{l}}$ and $s_{2}:=\sum_{l=0}^{\infty} b_{l} t^{p^{l}}$, where $a_{l}, b_{l} \in \mathbb{F}_{p}$ are chosen such that $t, s_{1}, s_{2}$ are algebraically independent. In this case we also have a purely inseparable PPV-extension of degree $p^{2}$, namely $E=F\left(r_{1}, r_{2}\right) \subseteq K((t))$ with $r_{1}^{p}=$ $s_{1}-a_{0} t, r_{2}^{p}=s_{2}-b_{0} t . r_{1}$ is a solution of the IDE

$$
\theta^{\left(p^{l}\right)}\left(\begin{array}{cc}
1 & r_{1} \\
0 & 1
\end{array}\right)=\left(\begin{array}{cc}
0 & a_{l+1} \\
0 & 0
\end{array}\right)\left(\begin{array}{cc}
1 & r_{1} \\
0 & 1
\end{array}\right) \quad(l \in \mathbb{N})
$$

and $r_{2}$ is a solution of the IDE with $a_{l+1}$ replaced by $b_{l+1}$. Hence the Galois group scheme - a subgroup scheme of $\mathbb{G}_{a} \times \mathbb{G}_{a}$ - is equal to $\alpha_{p} \times \alpha_{p}$ (where $\alpha_{p}$ denotes the Frobenius kernel inside $\mathbb{G}_{a}$ ).

In this case, there are infinitely many intermediate $\theta$-fields, since $\alpha_{p} \times \alpha_{p}$ has infinitely many subgroups which are given by the ideals $\left(a y_{1}+b y_{2}\right) \unlhd$ $K\left[y_{1}, y_{2}\right] /\left(y_{1}^{p}, y_{2}^{p}\right):=K\left[\alpha_{p} \times \alpha_{p}\right](a, b \in K)$.

The action is given by $\rho: R \rightarrow R \otimes_{K} K\left[y_{1}, y_{2}\right] /\left(y_{1}^{p}, y_{2}^{p}\right)$ with $\rho\left(r_{i}\right)=$ $r_{i} \otimes 1+1 \otimes y_{i}(i=1,2)$.

So the corresponding intermediate $\theta$-fields are $F\left(a r_{1}+b r_{2}\right), a, b \in K$.

Comparing this example with the one before, we see that - even for finite extensions - the Galois group scheme depends on the iterative derivation. This is contrary to finite separable PPV-extensions, where the Galois group is already determined by the extension of fields itself (cf. [Mat01, Thm. $1.15)$.

\section{Finite inseparable extensions}

In this section we compare our results for finite purely inseparable PPV-extensions with the Galois theory for purely inseparable field extensions given by Chase in Cha76.

So let us first give a brief overview on some results in Cha76]: Let $E / F$ be a purely inseparable field extension. Then the group functor

$$
G_{t}(E / F):(\text { TruncAlg } / F) \rightarrow(\text { Groups }), L \mapsto \operatorname{Aut}\left(E \otimes_{F} L / L\right)
$$

from the category of truncated $F$-algebras (i.e. algebras of the form $F\left[t_{1}, \ldots, t_{r}\right] /$ $\left.\left(t_{1}^{n_{1}}, \ldots, t_{r}^{n_{r}}\right)\right)$ to the category of groups is representable by a truncated $F$-algebra $U$. If the extension $E / F$ is modular (i.e. for all $i \in \mathbb{N}, E^{p^{i}}$ and $F$ are linearly disjoint over $\left.E^{p^{i}} \cap F\right)$, then $E^{G_{t}(E / F)}=F$ and $\operatorname{dim}_{F}(U)=[E: F]^{[E: F]}$. In this case, there is a Galois correspondence between the intermediate fields $F \leq M \leq E$, s.t. $E / M$ is modular, and certain closed subgroup schemes of $G_{t}(E / F)$, given in the usual way by taking fixed fields, respectively subgroups, fixing the given intermediate field. Furthermore, he showed that a purely inseparable field extension $E / F$ is modular if and only if there exists a truncated group scheme $\mathcal{G}$ (i.e. an affine group scheme represented by a truncated $F$-algebra) which acts on $E / F$, s.t. $\operatorname{Spec}(E)$ is a $\mathcal{G}$-torsor. Given such a group scheme $\mathcal{G}$, then $G_{t}(E / F) \cong \mathcal{G}\left(E \otimes_{F}-\right)$ as truncated group schemes over $F$. However, although the group scheme $G_{t}(E / F)$ is unique, there might be several such group schemes $\mathcal{G}$. 
Now return to the case where $E / F$ is a purely inseparable PPV-extension and $\mathcal{G}:=\underline{\operatorname{Gal}}(E / F)$. By Corollary 11.6, $\mathcal{G}$ is infinitesimal, and since $K$ is perfect, $K[\mathcal{G}]$ is a truncated $K$-algebra (cf. [DG70], III, $\S 3$, Cor. 6.3 ) and so $F[\mathcal{G}$ ] is a truncated $F$-algebra. As shown in Corollary 10.11, $E$ is a $\mathcal{G}_{F}$-torsor.

By the statements above, we obtain that $E / F$ is a modular field extension and that $G_{t}(E / F)$ equals $\mathcal{G}_{F}\left(E \otimes_{F}-\right)$. So we can regain the truncated Galois group scheme $G_{t}(E / F)$ from our Galois group scheme $\operatorname{Gal}(E / F)$.

However, starting with $G_{t}(E / F)$, the iterable higher derivation leads to a natural choice for a group scheme $\mathcal{G}_{F} \leq G_{t}(E / F)$ over which $E$ is a torsor (namely $\left.\operatorname{Gal}(E / F)_{F}\right)$ and also gives a natural description of the intermediate fields corresponding to the closed subgroup schemes of $\mathcal{G}$. For instance, in Example 11.8]i), iii), $F=K\left(t, s_{1}, s_{2}\right)$ is the rational function field in three variables and $E / F$ is a purely inseparable field extension of degree $p^{2}$ and exponent 1 . Hence in both examples we have the same (abstract) field extension. But in one case the iterable higher derivation leads to the Galois group scheme $\operatorname{Gal}(E / F)=\alpha_{p} \times \alpha_{p}$ and in the other case to $\operatorname{Gal}(E / F)=\mu_{p} \times \mu_{p}$. Other iterable higher derivations would also lead to different Galois group schemes. The truncated Galois group scheme $G_{t}(E / F)$ only gives a bound on which Galois group schemes $\mathrm{Gal}(E / F)$ may occur, because every one of them will be a closed subgroup scheme of $G_{t}(E / F)$.

\section{REFERENCES}

[AM05] Amano, K.. Masuoka, A.: Picard-Vessiot extensions of Artinian simple module algebras. Journal of Algebra, 285:743-767 (2005) MR2125463 (2005m:16046)

[Ama07] Amano, K.: On a discrepancy among Picard-Vessiot theories in positive characteristics. eprint arXiv:math/0612683v2 (2007)

[And01] André, Y.: Différentielles non commutatives et théorie de Galois différentielle ou aux différences. Ann. Scient. Éc. Norm. Sup., (4), 34:685-739 (2001) MR.1862024 (2002k:12013)

[BO78] Berthelot, P.. Ogus, A.: Notes on crystalline cohomology. Princeton University Press (1978) MR0491705 (58:10908)

[Cha76] Chase, S.U.: Infinitesimal group scheme actions on finite field extensions. Am. J. Math., (2) 98:441-480 (1976) MR0424773 (54:12731)

[Del90] Deligne, P.: Catégories Tannakiennes, in Grothendieck Festschrift, Vol II. Birkhäuser Boston, pp. 111-195 (1990) MR.1106898(92d:14002)

[DG70] Demazure, M.. Gabriel, P.: Groupes Algébriques. Tome I: Géométrie algébrique, généralités, groupes commutatifs. North-Holland Pub. Comp., Amsterdam (1970) MR0302656 (46:1800)

[DM89] Deligne, P.. Milne, J.: Tannakian Categories, in Hodge Cycles, Motives and Shimura Varieties. Springer Lecture Notes 900:101-228 (1989)

[Dyc08] Dyckerhoff, T.: The Inverse Problem of Differential Galois Theory over the Field $\mathbb{R}(t)$. eprint arXiv:0802.2897v1 (2008)

[Gro64] Grothendieck, A.: Éléments de géométrie algébrique IV. Publ. Math. de l'I. H. É. S. 20 (1964)

[Eis95] Eisenbud, D.: Commutative Algebra with a View Toward Algebraic Geometry. Graduate Texts in Mathematics 150, Springer (1995) MR1322960(97a:13001)

[Gie75] Gieseker, D.: Flat vector bundles and the fundamental group in non-zero characteristics. Ann. Scuola Norm. Sup. Pisa Cl. Sci. (4), (2)1:1-31 (1975) MR0382271 (52:3156)

[Hard08] Hardouin, C.: Iterative q-difference Galois Theory. IWR-Preprint (2008) (available at http://www.ub.uni-heidelberg.de/archiv/8278/ )

[Hart77] Hartshorne, R.: Algebraic Geometry. Graduate Texts in Mathematics 52, Springer (1977) MR0463157 (57:3116)

[HS37] Hasse, H.. Schmidt, F.K.: Noch eine Begründung der Theorie des höheren Differentialquotienten in einem algebraischen Funktionenkörper in einer Unbestimmten. J. Reine Angew. Math. 177:215-237 (1937) 
[Hei07] Heiderich, F.: Picard-Vessiot-Theorie für lineare partielle Differentialgleichungen. Heidelberg University Library, Diplom thesis (2007)

[Jac64] Jacobson, N.: Lectures in Abstract Algebra, Vol III: Theory of Fields and Galois Theory. Van Nostrand, Princeton, NJ (1964); Springer-Verlag reprint (1975) MR0172871 $(30: 3087)$

[Jan03] Jantzen, J.C.: Representations of algebraic groups. Math. Surveys and Monographs, 107, Am. Math. Soc. (2003) MR2015057 (2004h:20061)

[Kat70] Katz, N.: Nilpotent connections and the monodromy theorem: Applications of a result of Turrittin. Publ. Math. de l'I. H. É. S. 39:175-232 (1970) MR0291177 (45:271)

[Kat87] Katz, N.: On the calculation of some differential Galois groups. Invent. Math. 87:13-61 (1987) MR862711 (88c:12010)

[Mat01] Matzat, B.H.: Differential Galois Theory in Positive Characteristic, notes written by J. Hartmann. IWR-Preprint 2001-35 (2001) (available at http://www.iwr.uniheidelberg.de/ Heinrich.Matzat/publications.html)

[Mats89] Matsumura, H.: Commutative ring theory. Cambridge Studies in Adv. Math. 8 (1989) MR 1011461 (90i:13001)

[MvdP03] Matzat, B.H.. van der Put, M.: Iterative differential equations and the Abhyankar conjecture. J. Reine Angew. Math. 557:1-52 (2003) MR.1978401 (2004d:12011)

[Pap08] Papanikolas, M.: Tannakian duality for Anderson-Drinfeld motives and algebraic independence of Carlitz logarithms. Invent. Math. 171:123-174 (2008) MR2358057

[Rös07] Röscheisen, A.: Iterative Connections and Abhyankar's Conjecture. Heidelberg University Library, Ph.D. thesis (2007) (available at http://www.ub.uniheidelberg.de/archiv/7179/)

[San07] Dos Santos, J.P.: Fundamental group schemes for stratified sheaves. Journal of Algebra 317:691-713 (2007) MR 2362937 (2008h:14045)

[Tak89] Takeuchi, M.: A Hopf Algebraic Approach to the Picard-Vessiot Theory. Journal of Algebra 122:481-509 (1989) MR999088 (90j:12016)

[vdPS03] van der Put, M.. Singer, M.F.: Galois Theory of Linear Differential Equations. Grundlehren Math. Wiss. 328, Springer (2003) MR1960772 (2004c:12010)

[Voj07] Vojta, P.: Jets via Hasse-Schmidt Derivations. In Diophantine Geometry, Proceedings, pp. 335-361. Edizioni della Normale, Pisa (2007) MR2349665 (2008i:13043)

Interdisciplinary Center for Scientific Computing, Heidelberg University, Im Neuenheimer Feld 368, 69120 Heidelberg, Germany

E-mail address: andreas.maurischat@iwr.uni-heidelberg.de 